\title{
$12 \mathrm{GeV}$ upgrade of Jefferson Lab
}

Franck Sabatie $\quad$ Focus: the Generalized Parton Distribution program CEA Saclay

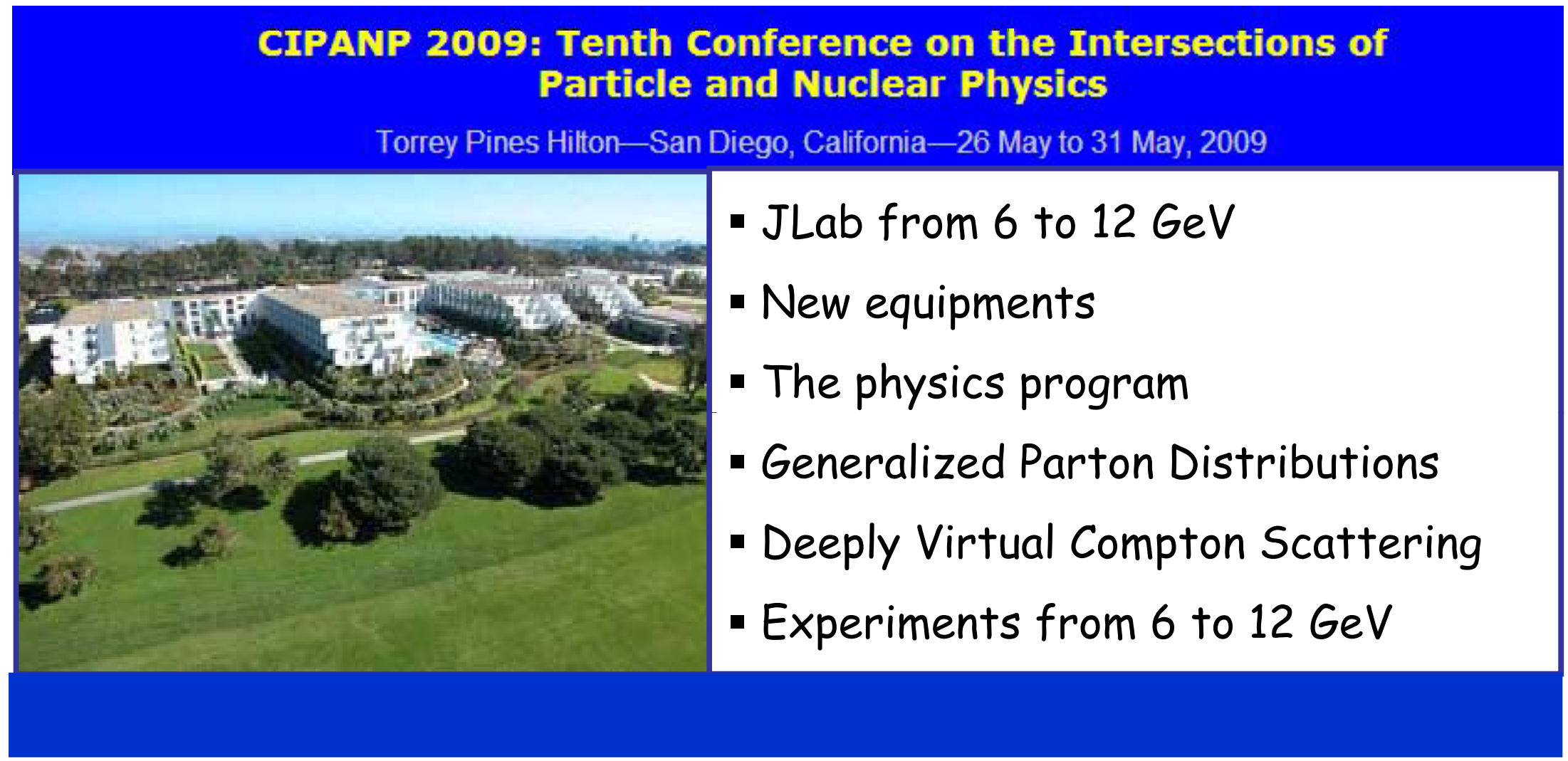




\section{Jefferson Lab today}

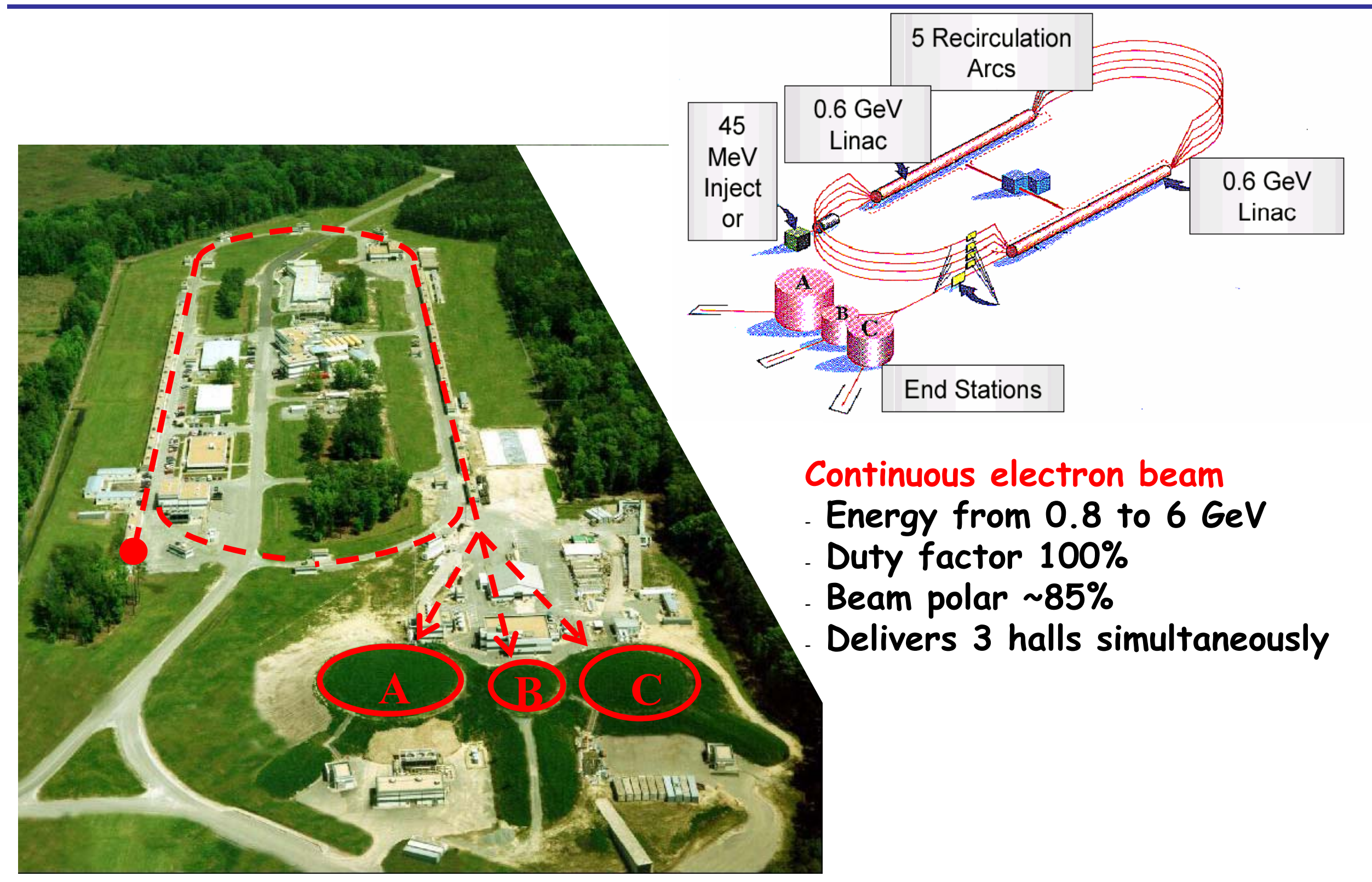




\section{Upgrading JLab from 6 to $12 \mathrm{GeV}$}

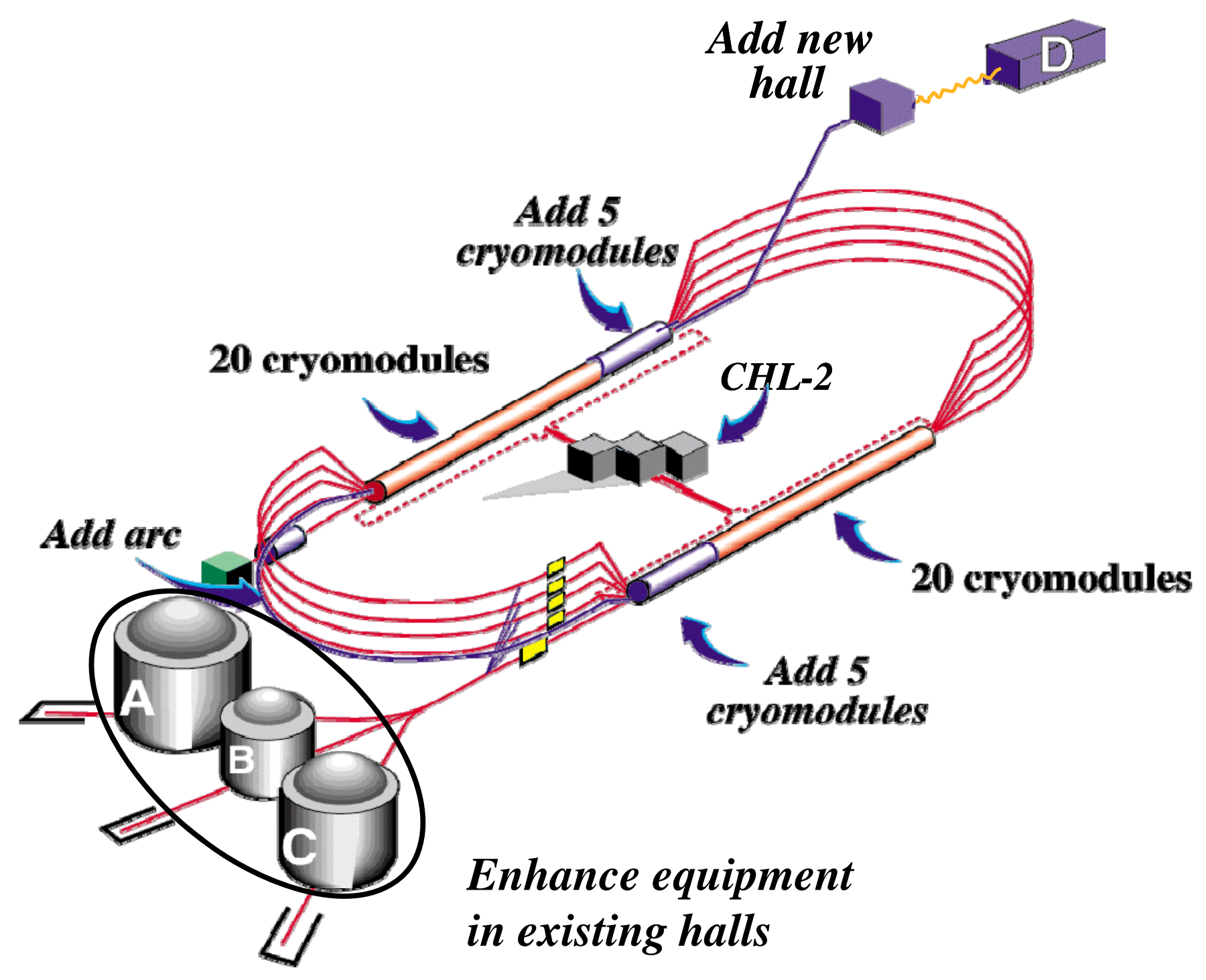




\section{New equipments for 11-12 GeV beam}

D

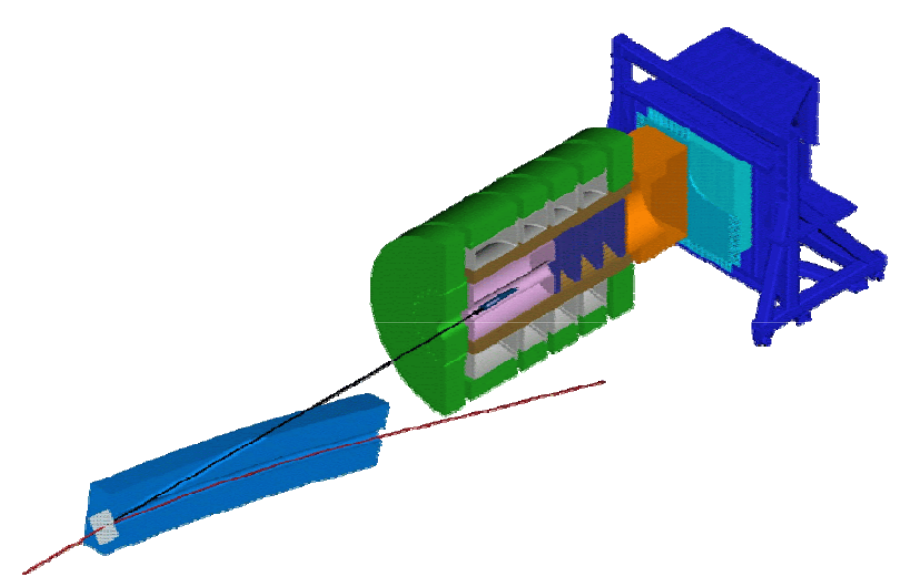

$9 \mathrm{GeV}$ tagged polarized photons and a $4 \pi$ hermetic detector

B

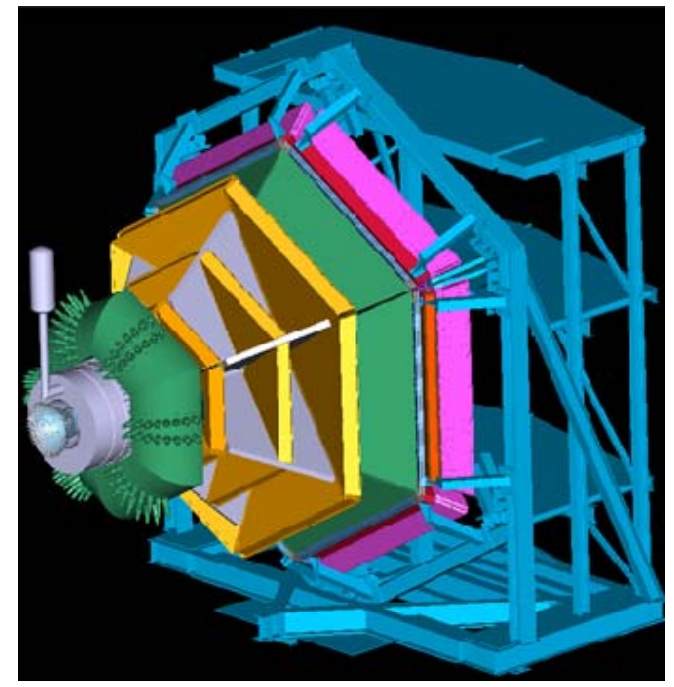

CLAS12 with new detectors and higher luminosity $\left(10^{35} / \mathrm{cm}^{2}\right.$-s $)$
C

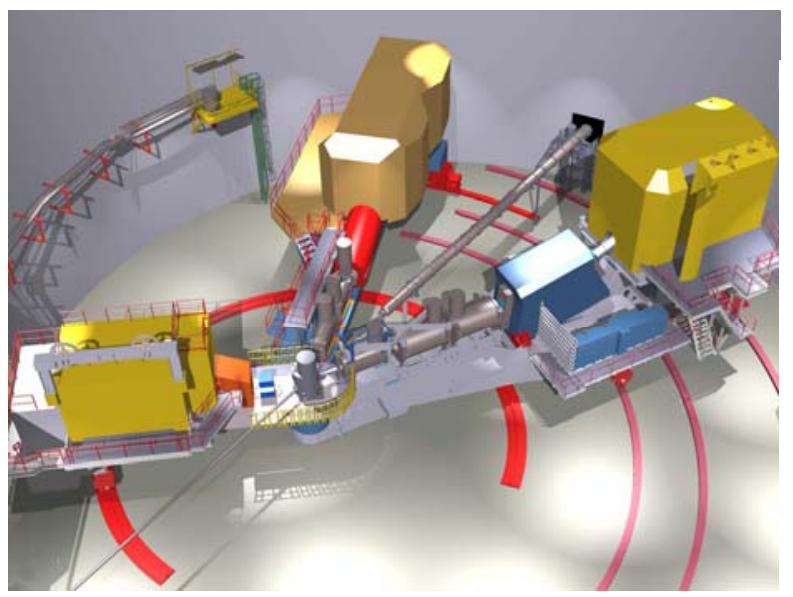

Super High Momentum Spectrometer (SHMS) at high luminosity and forward angles

A

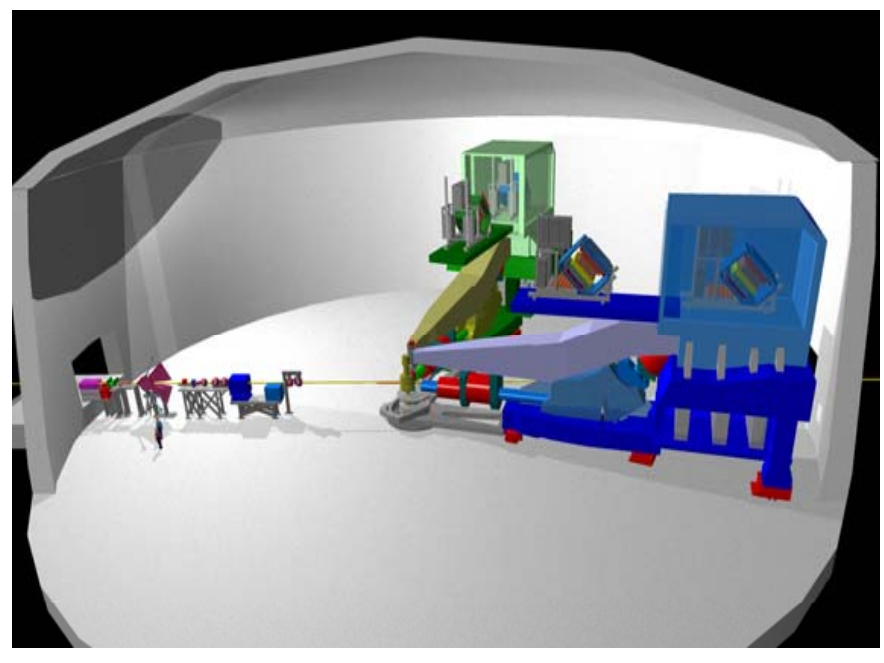

High Resolution Spectrometer (HRS) Pair, and specialized large installation experiments 


\section{... and construction has started!}

Now at: Critical Decision Three (CD-3)

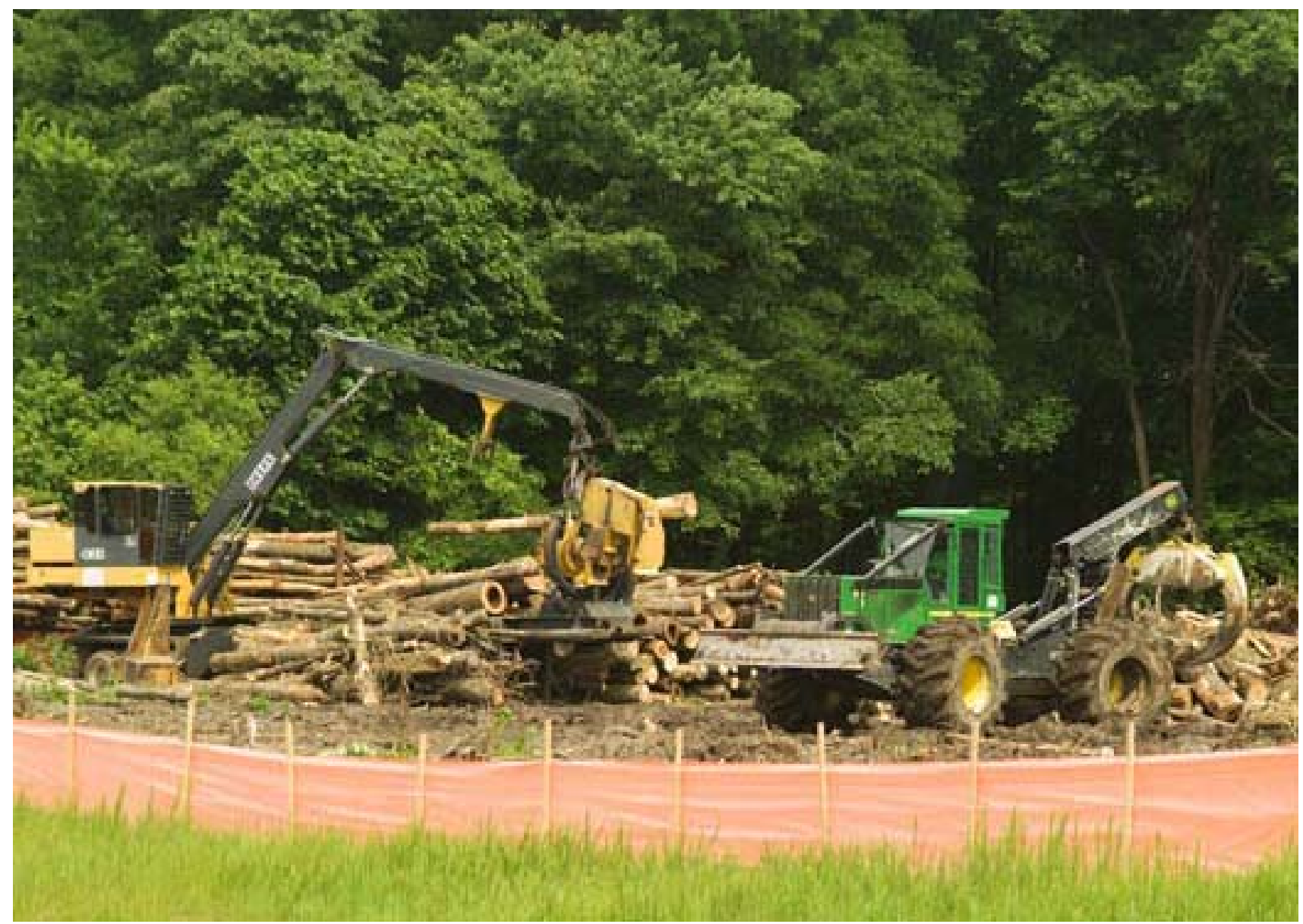

Making Progress - Construction crews have started to remove trees as work on the $\$ 310$ million $12 \mathrm{GeV}$ Upgrade gains momentum. Hall D, the lab's fourth experimental hall, will be constructed in the cleared area. The upgrade is scheduled for completion in 2015. 


\section{Physics topics with 12 GeV Jefferson Lab}

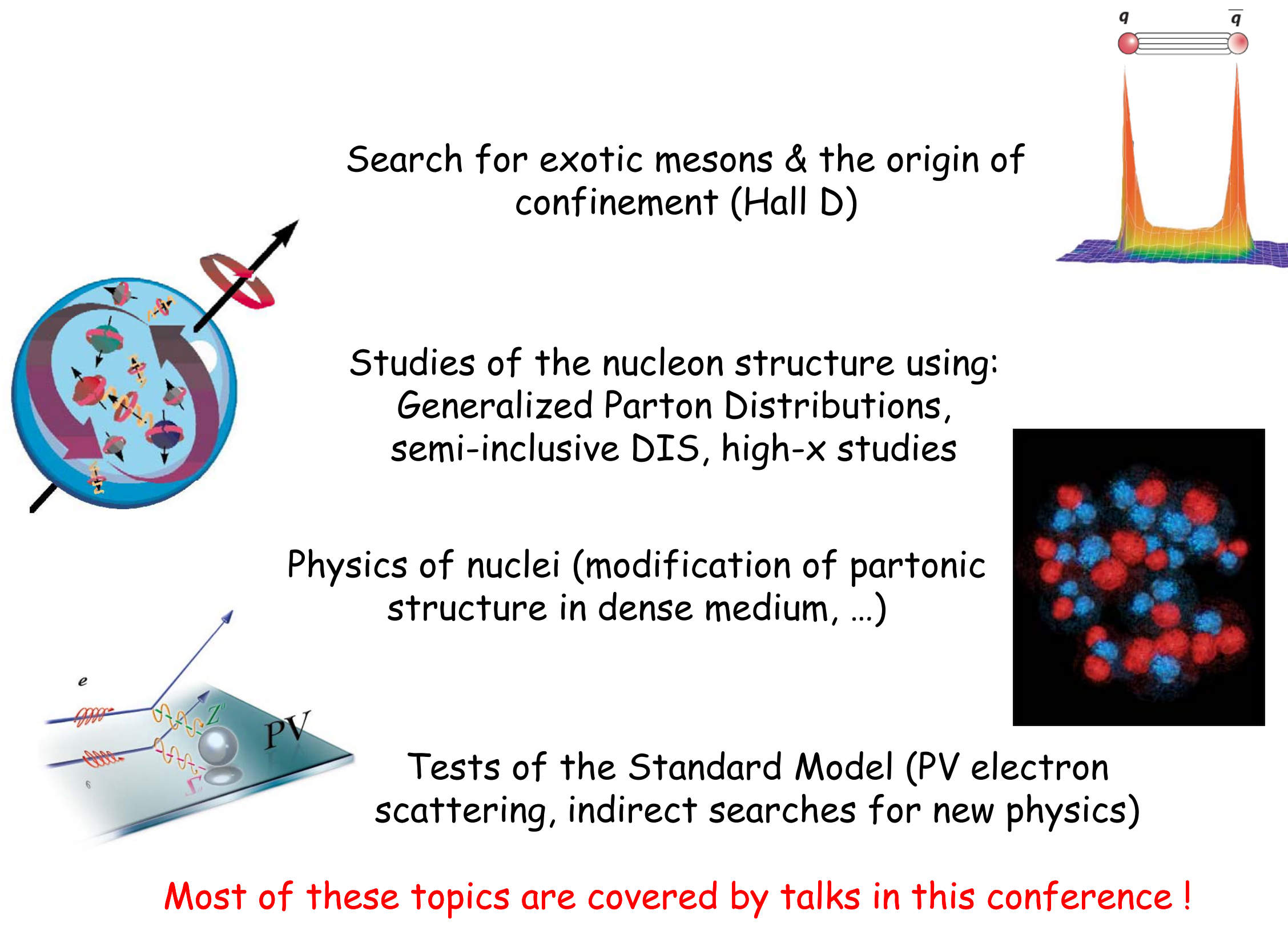




\section{Physics topics with 12 GeV Jefferson Lab}

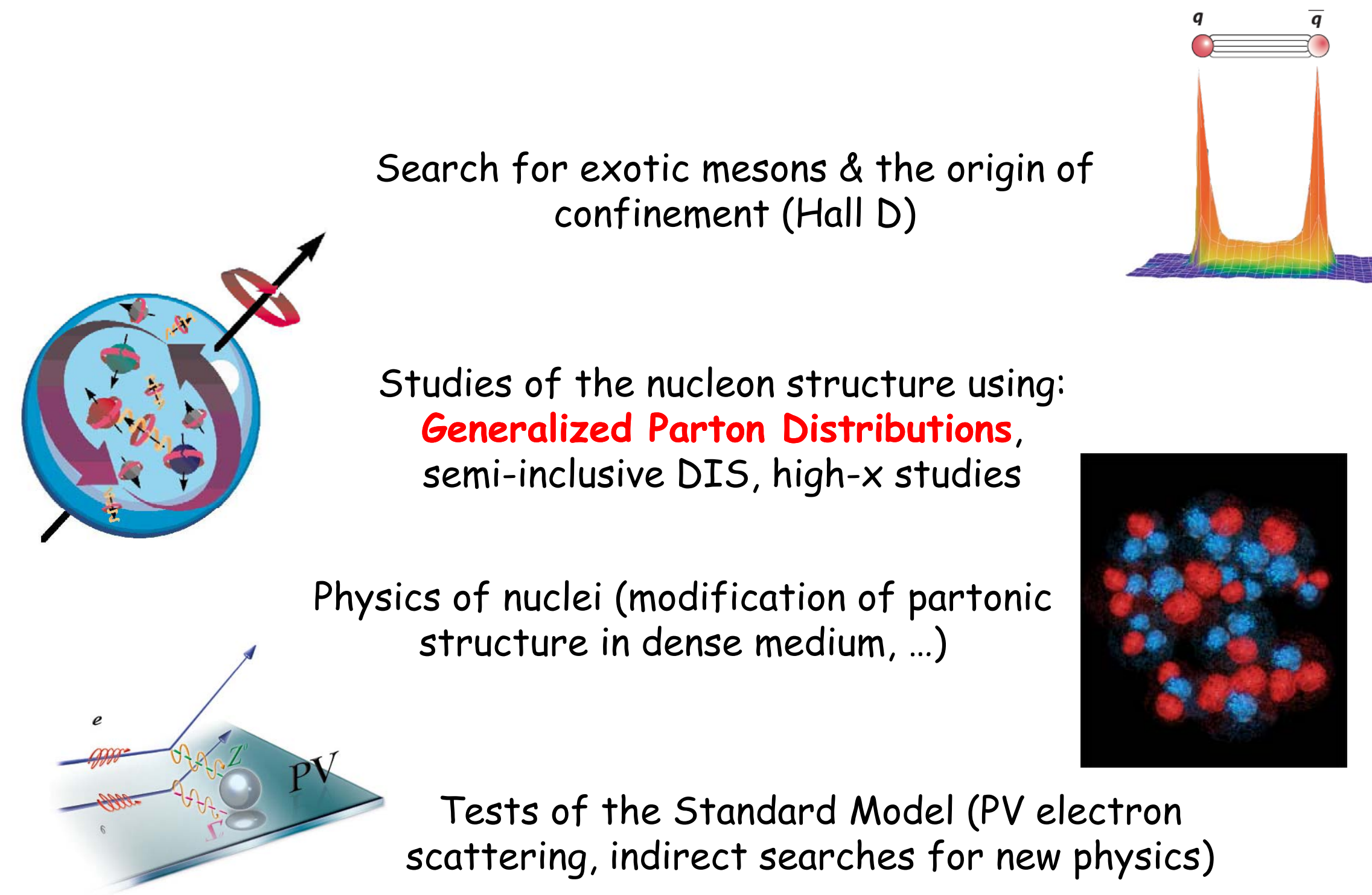


The electromagnetic probe for the nucleon structure

Local, Off-diagonal

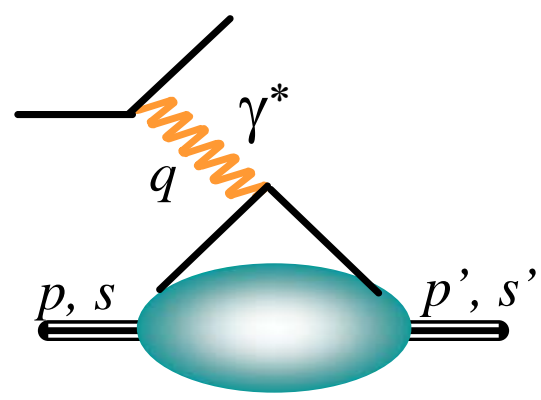

Hofstadter (1958)

$$
\begin{aligned}
& \left\langle p^{\prime} s^{\prime}\left|\bar{\psi}(0) \gamma^{\mu} \psi(0)\right| p s\right\rangle \rightarrow G_{M}, G_{E} \\
& \left\langle p^{\prime} s^{\prime}\left|\bar{\psi}(0) \gamma^{\mu} \gamma^{5} \psi(0)\right| p s\right\rangle \rightarrow G_{A}, G_{P}
\end{aligned}
$$

Non-local, Diagonal

\section{Deep Inelastic Scattering}

Taylor et al. (1969)
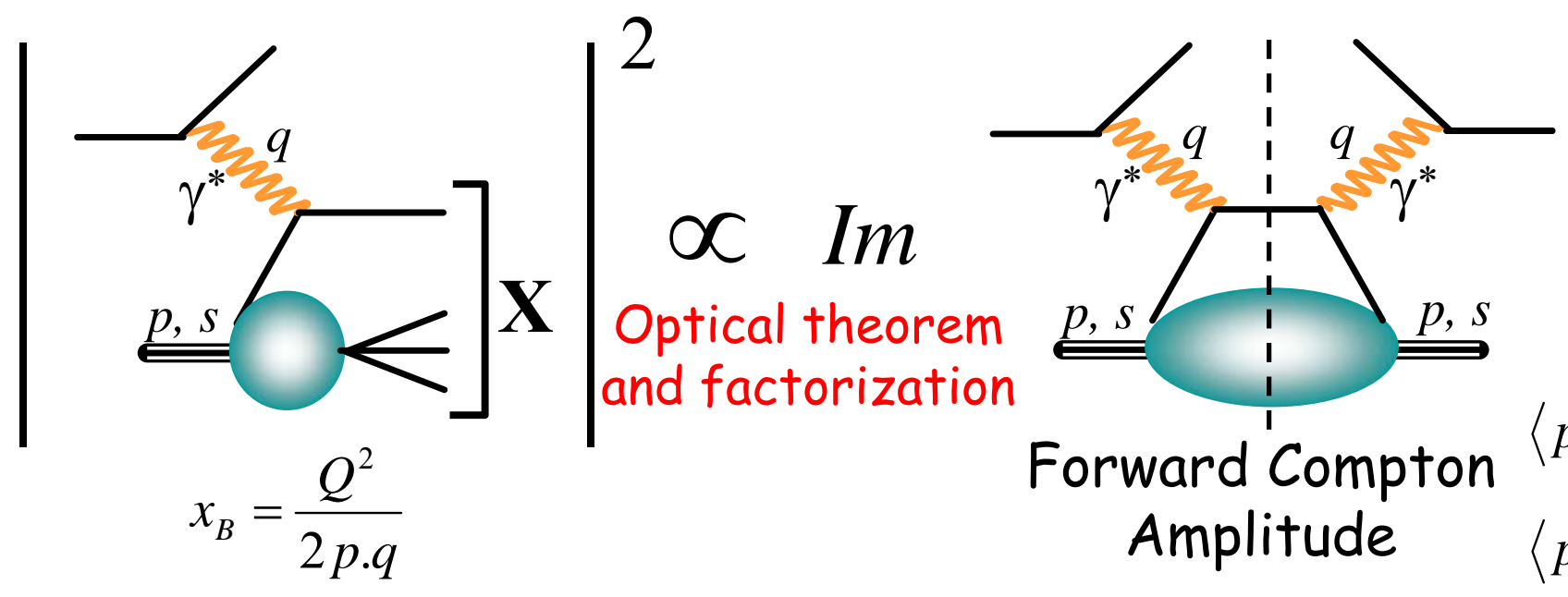

Forward 'Compton

Amplitude

$\left\langle p s\left|\bar{\psi}(-y / 2) \gamma^{\mu} \psi(y / 2)\right| p s\right\rangle \rightarrow F_{2}$

$\left\langle p s\left|\bar{\psi}(-y / 2) \gamma^{\mu} \gamma^{5} \psi(y / 2)\right| p s\right\rangle \rightarrow g_{1}$ 
A natural extension: non-local, off-diagonal matrix elements

Mueller

(1995)

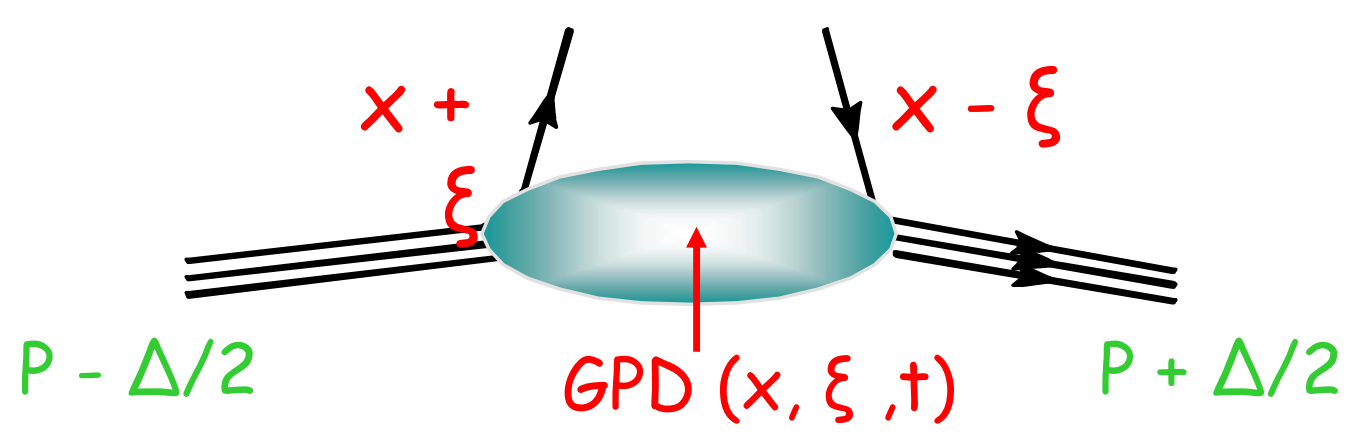

$(x+\xi)$ and $(x-\xi)$ : longitudinal momentum fractions of quarks

The structure of the nucleon can be described by

4 Generalized Parton Distributions:
Vector: $H(x, \xi, t)$
Axial-Vector : $\tilde{H}(x, \xi, t)$
Tensor: $E(x, \xi, t)$
Pseudoscalar : $\tilde{E}(x, \xi, t)$ 
And its golden process : Deeply Virtual Compton Scattering

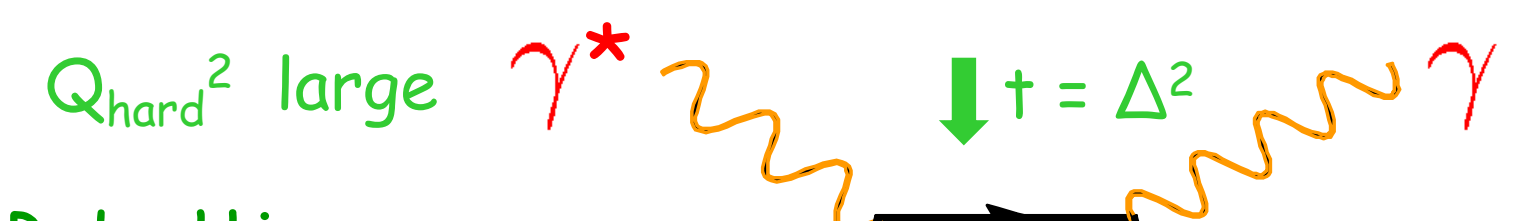

Ji, Radyushkin

(1996)

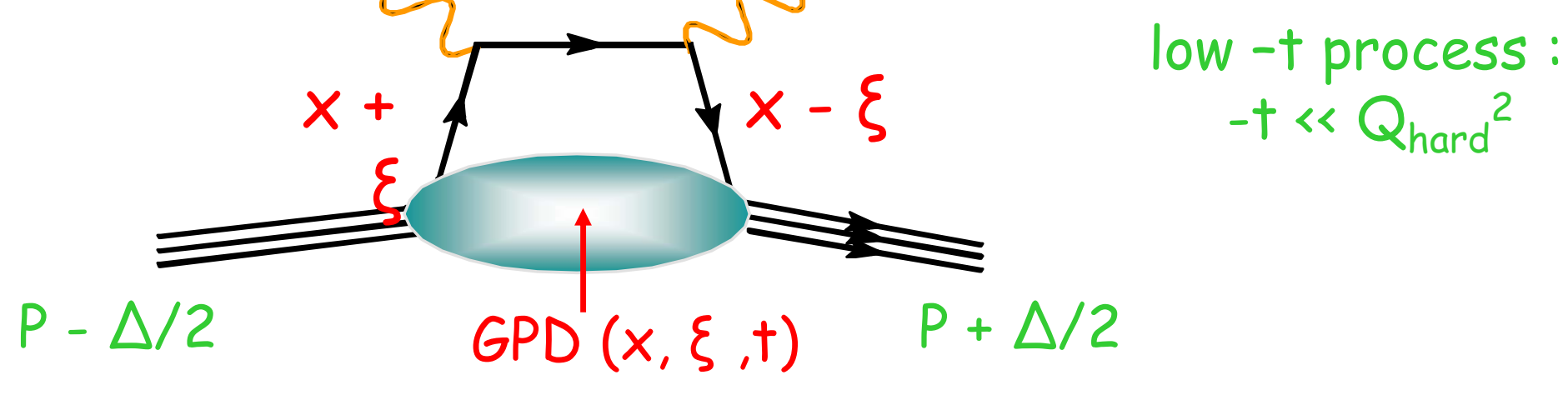

$(x+\xi)$ and $(x-\xi)$ : longitudinal momentum fractions of quarks

at large $Q^{2}: Q C D$ factorization theorem $\Rightarrow$ hard exclusive processes can be described by 4 Generalized Parton Distributions:
Vector: $H(x, \xi, t)$
Axial-Vector : $\tilde{H}(x, \xi, t)$
Tensor: $E(x, \xi, t)$
Pseudoscalar : $\tilde{E}(x, \xi, t)$ 
Why Generalized Parton Distributions are the way to go!

\section{GPDs yield 3-dim quark \\ structure of the nucleon}

* v
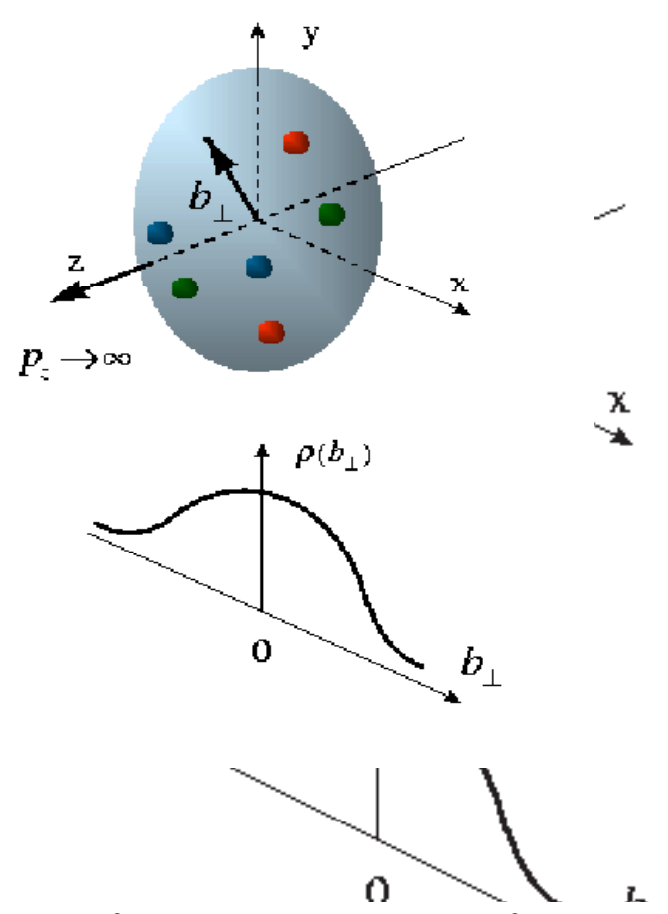

Elastic Scattering transverse quark distribution in coordinate space

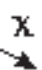

Burkardt (2000, 2003)

Belitsky, Ji, Yuan (2003)
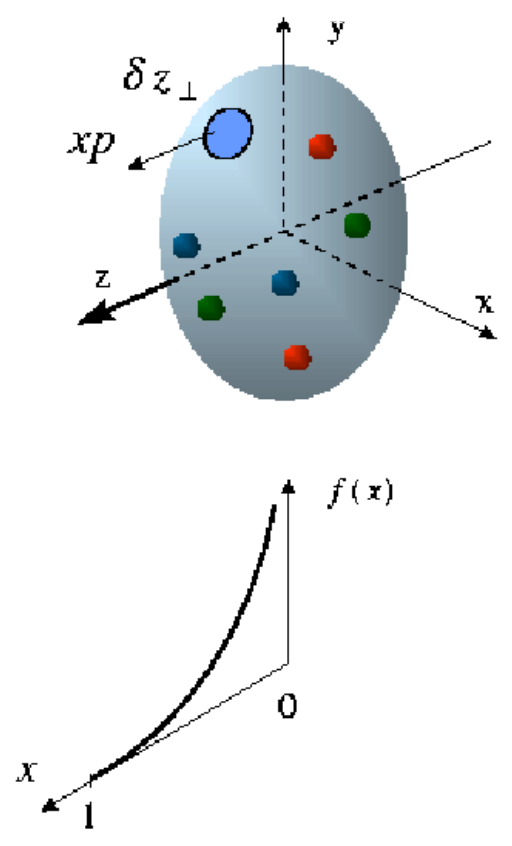

DIS

longitudinal

quark distribution in momentum space

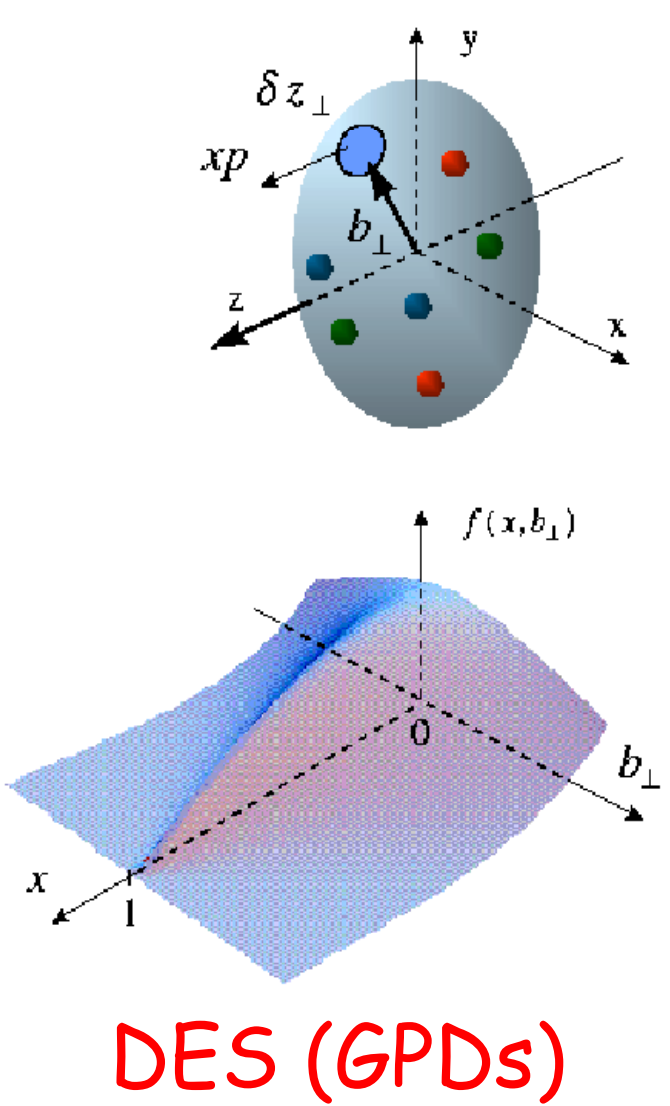

fully-correlated quark distribution in both coordinate and momentum space 


\section{Properties, applications}

They contain what we know already through sum rules and kinematical limits:

Form Factors, parton distributions

$>$ forward limit : ordinary parton distributions

$H^{q}(x, \xi=0, t=0)=q(x) \quad$ unpolarized quark distributions

$\tilde{H}^{q}(x, \xi=0, t=0)=\Delta q(x)$ polarized quark distributions

$E^{q}, \tilde{E}^{q}$ : do NOT appear in DIS ... new information

$>$ first moments : nucleon electroweak form factors

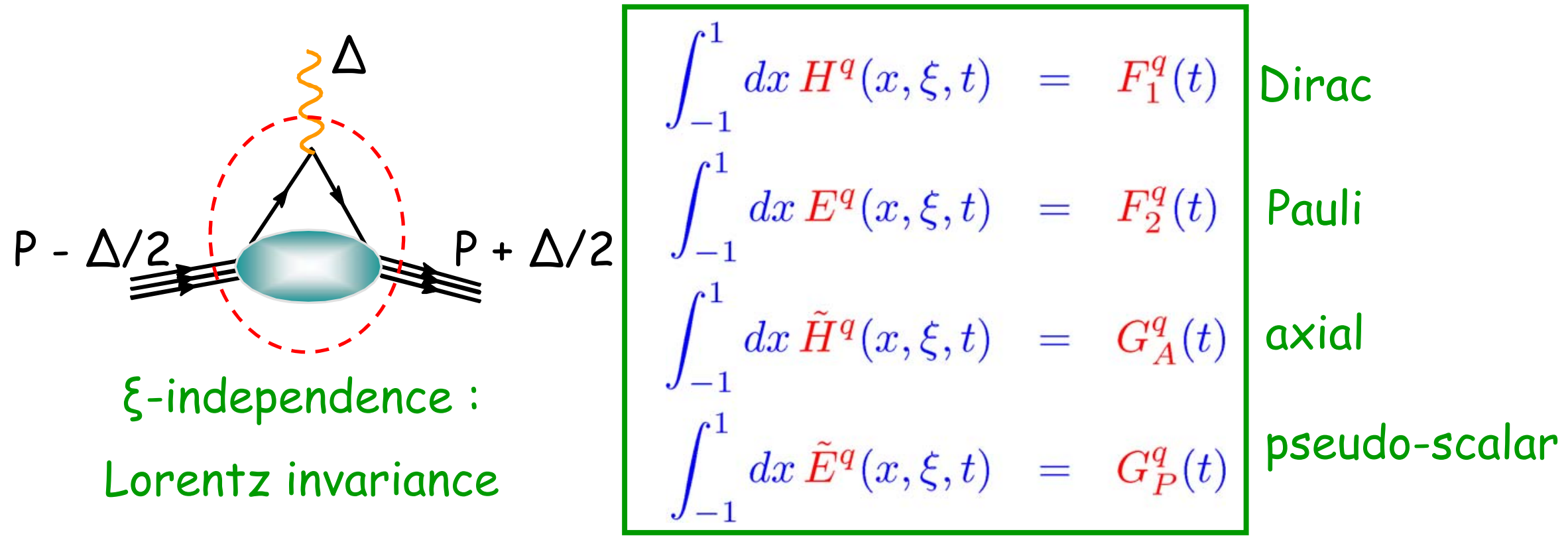




\section{Properties, applications}

They contain what we know already through sum rules and kinematical limits:

Form Factors, parton distributions

Through the space-momentum correlation, they give access to the

Orbital Angular Momentum (OAM) carried by partons inside the nucleon: Finally an end to the spin crisis?

Ji's sum rule:

$$
J^{q}=\frac{1}{2} \Delta \Sigma+L^{q}=\lim _{t \rightarrow 0} \frac{1}{2} \int_{-1}^{1} x d x\left[H^{q}(x, \xi, t)+E^{q}(x, \xi, t)\right]
$$

Moments of GPDs are calculable in Lattice $Q C D$. Lowest moments have already been computed for valence quarks.
Best accessible using transverse polarized target.

While waiting, neutron DVCS is sensitive to $E$ 


\section{Properties, applications}

They contain what we know already through sum rules and kinematical limits:

Form Factors, parton distributions

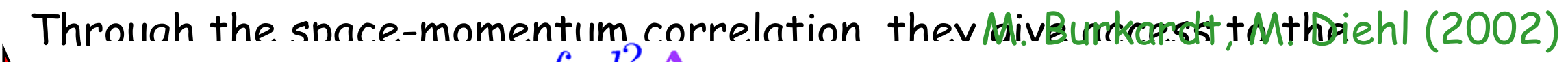

$\Rightarrow \underset{\text { nucl }}{\text { Ang }} H^{q}\left(x, \mathbf{b}_{\perp}\right)=\int \frac{d^{2} \Delta_{\perp}}{(2 \pi)^{2}} e^{i \mathbf{b}_{\perp} \cdot \Delta_{\perp}} H^{q}\left(x, \xi=0,-\Delta_{\perp}{ }^{2}\right)$

$\square$

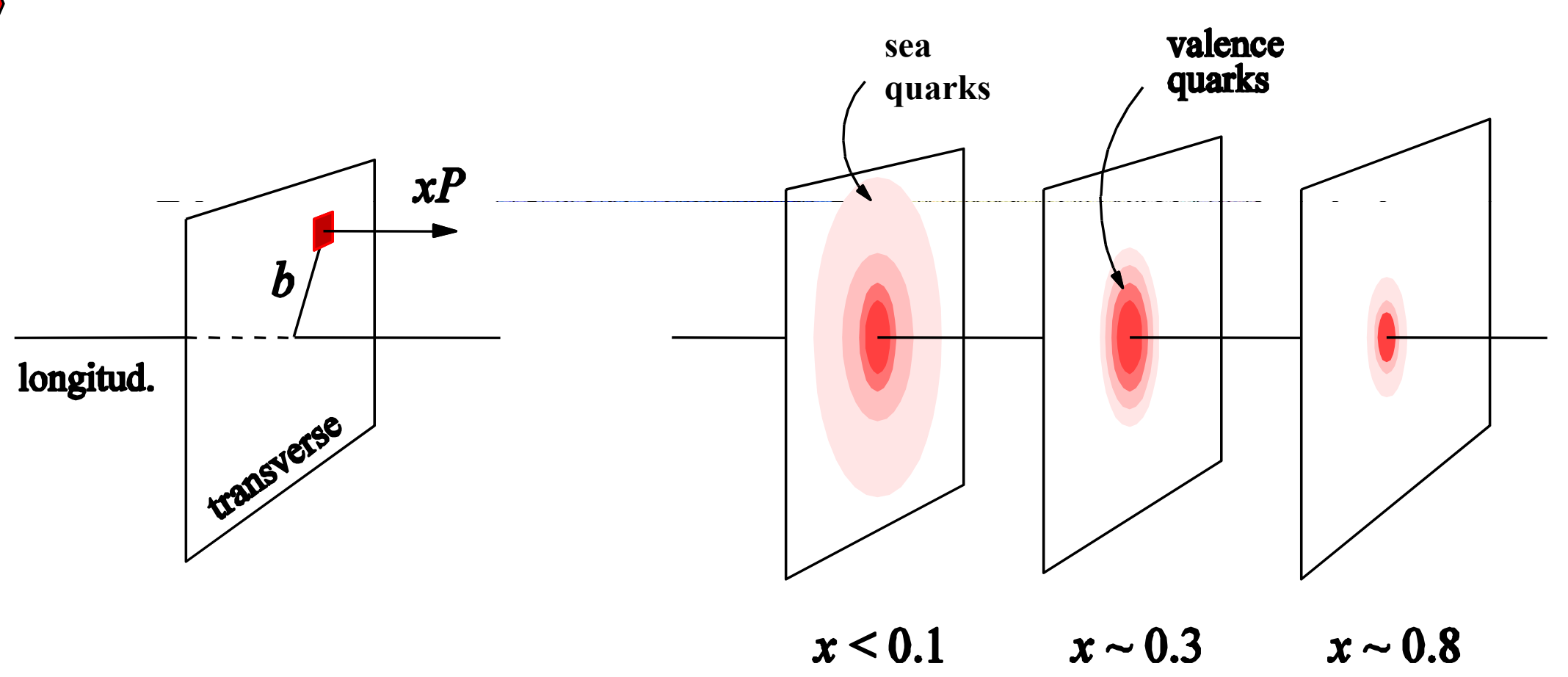




\section{D-imaging (full $\xi$ dependence needed !)}

GPDs as Wigner distribution can be used to picture quarks in the proton A. Belitsky, X. Ji, and F. Yuan (2003)

The associated Wigner distribution is a function of position $\vec{r}$ and Feynman momentum $x: f(\vec{r}, x)$

One can plot the Wigner distribution as a 3D function at fixed $x$ A GPD model satisfying known constraint:
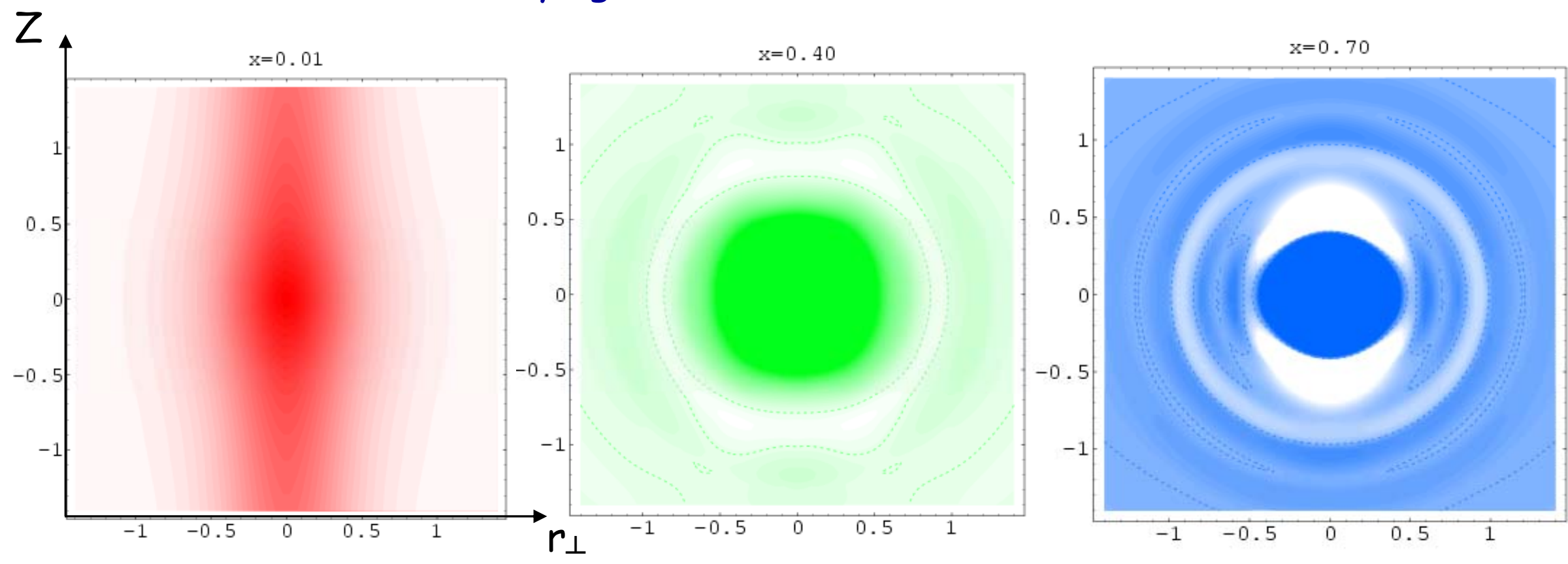


\section{GPDs from Theory to Experiment}

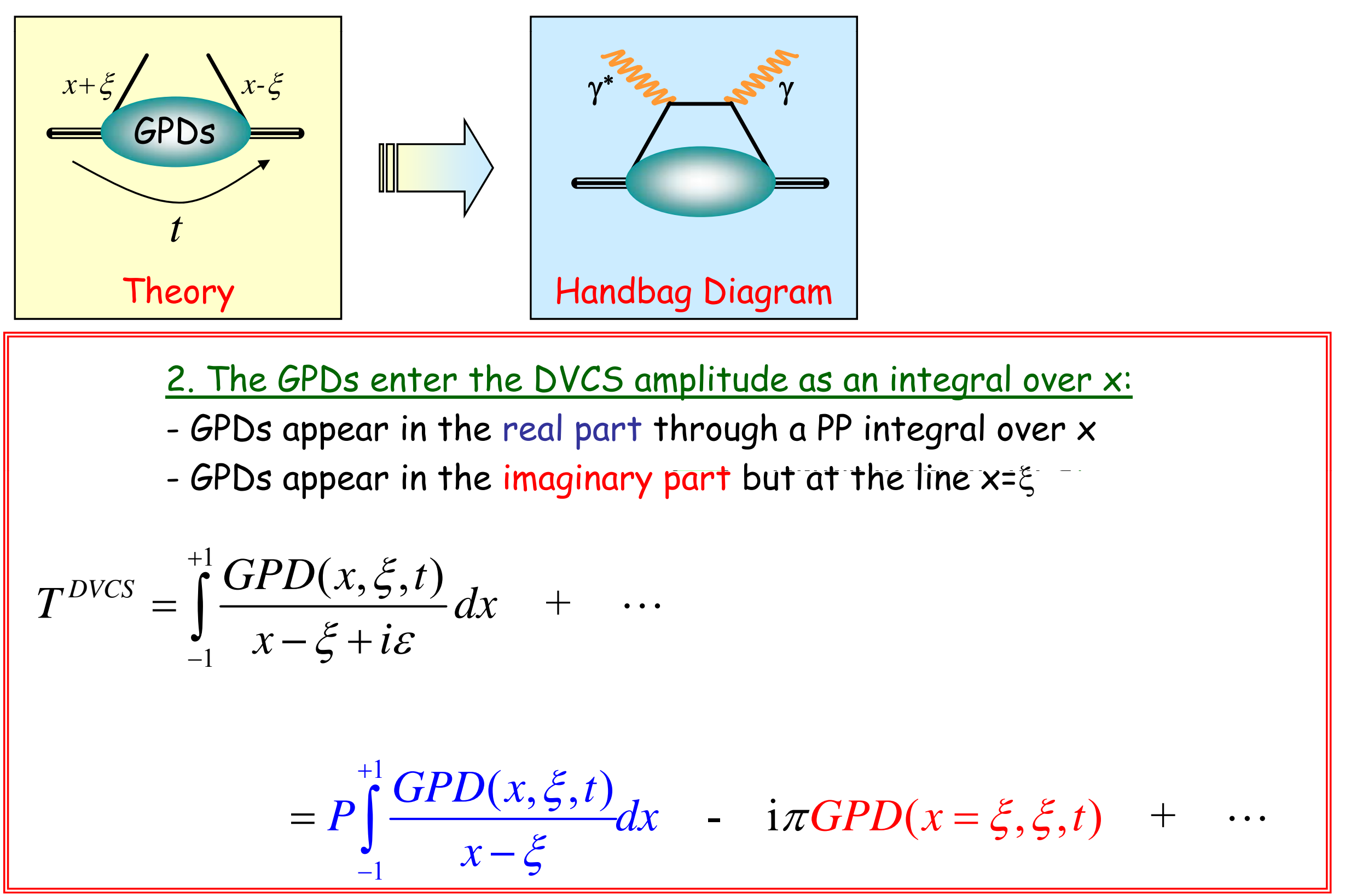




\section{Experimental observables linked to GPDs}

\section{Experimentally, DVCS is undistinguishable with Bethe-Heitler}

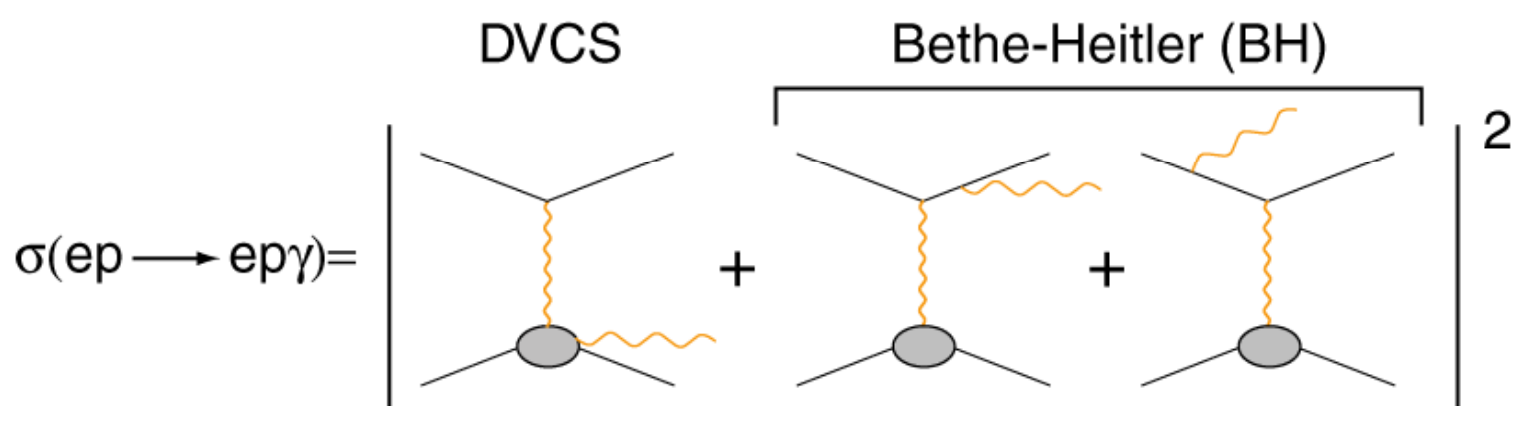

However, we know FF at low $t$ and $\mathrm{BH}$ is fully calculable

Using a polarized beam on an unpolarized target, 2 observables can be measured:

$$
\begin{aligned}
& \frac{d^{4} \sigma}{d x_{B} d Q^{2} d t d \varphi} \approx\left|T^{B H}\right|^{2}+2 T^{B H} \cdot \operatorname{Re}\left(T^{D V C S}\right)+\left|T^{D V C S}\right|^{2} \\
& \frac{d^{4} \vec{\sigma}-d^{4} \stackrel{\leftarrow}{\boldsymbol{d}}}{d x_{B} d Q^{2} d t d \varphi} \approx 2 T^{B H} \cdot \operatorname{Im}\left(T^{D V C S}\right)+\left[\left|T^{D V C S}\right|^{2}-\left|T^{D V C S}\right|^{2}\right]
\end{aligned}
$$




\section{Into the harmonic structure of DVCS}

$$
\begin{gathered}
\frac{d^{4} \sigma}{d x_{B} d Q^{2} d t d \varphi}=\frac{1}{\mathrm{P}_{1}(\varphi) \mathrm{P}_{2}(\varphi)} \Gamma_{1}\left(x_{B}, Q^{2}, t\right)\left\{c_{0}^{B H}+c_{1}^{B H} \cos \varphi+c_{2}^{B H} \cos 2 \varphi\right\}-\left|\mathrm{T}^{\mathrm{B} H}\right|^{2} \\
+\frac{1}{\mathrm{P}_{1}(\varphi) \mathrm{P}_{2}(\varphi)} \Gamma_{2}\left(x_{B}, Q^{2}, t\right)\left\{c_{0}^{I}+c_{1}^{I} \cos \varphi+c_{2}^{I} \cos 2 \varphi+c_{3}^{I} \cos 3 \varphi\right\} \\
\frac{d^{4} \vec{\sigma}-d^{4} \overleftarrow{\sigma}}{d x_{B} d Q^{2} d t d \varphi}=\frac{\Gamma\left(x_{B}, Q^{2}, t\right)}{\mathrm{P}_{1}(\varphi) \mathrm{P}_{2}(\varphi)} \frac{\left.s_{1}^{I} \sin \varphi+s_{2}^{I} \sin 2 \varphi\right\}}{\text { Interference term }}
\end{gathered}
$$

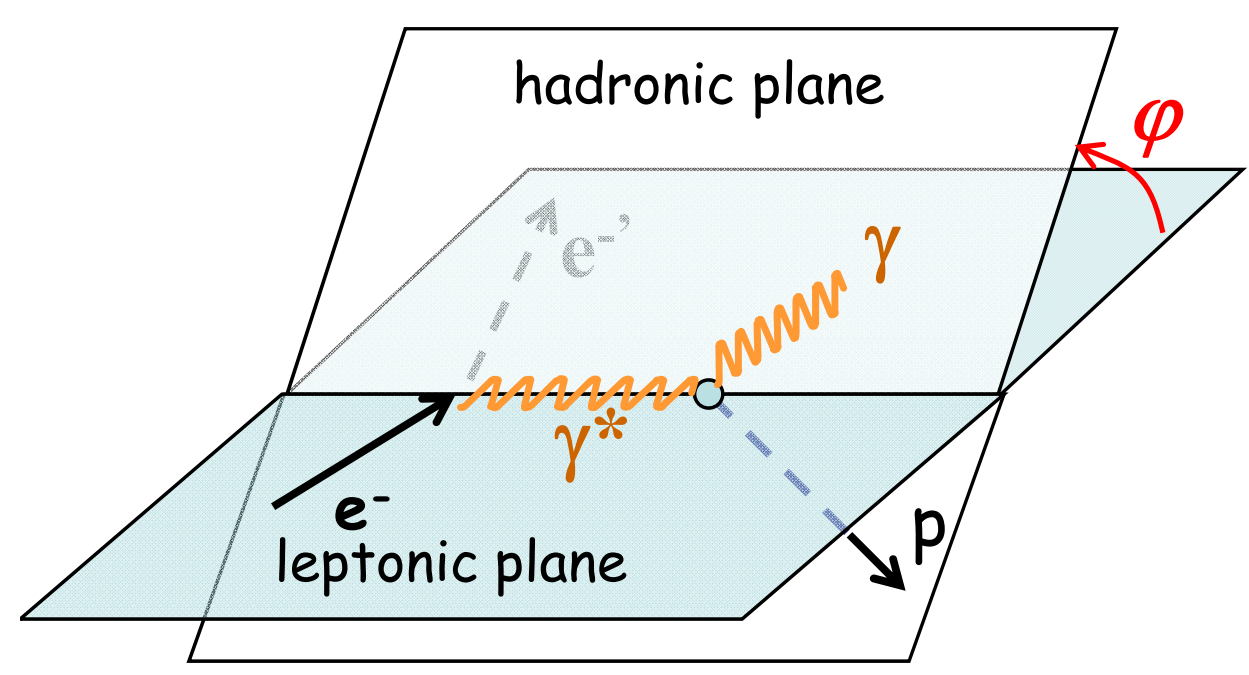

Belitsky, Mueller, Kirchner

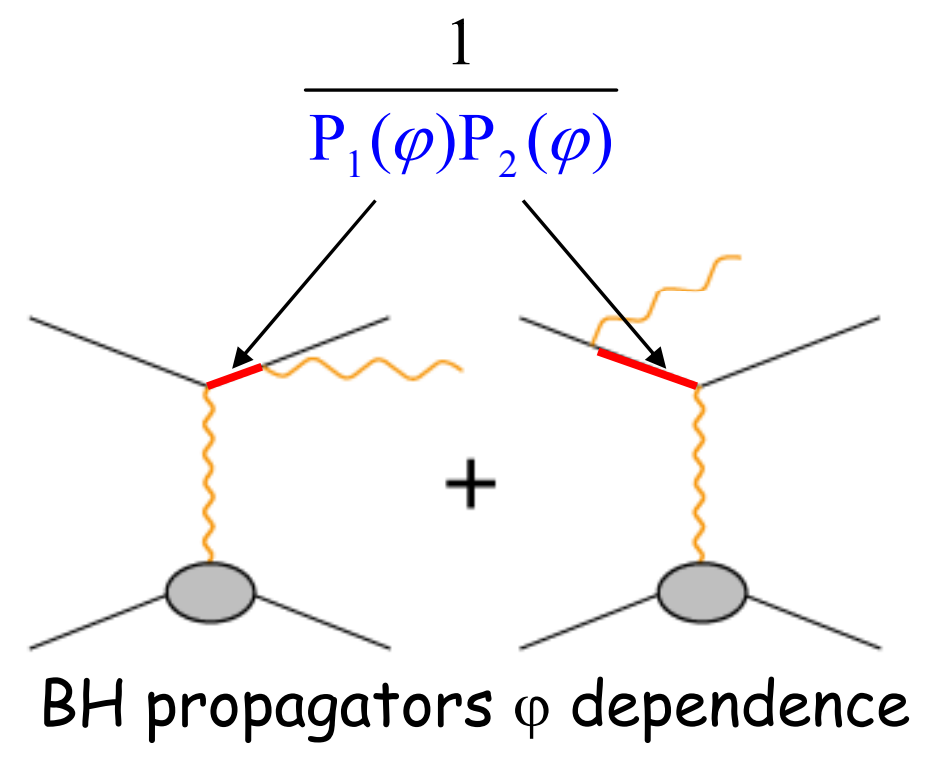

BH propagators $\varphi$ dependence 


\section{$6 \mathrm{GeV}$ breakthroughs and results in a nutshell}
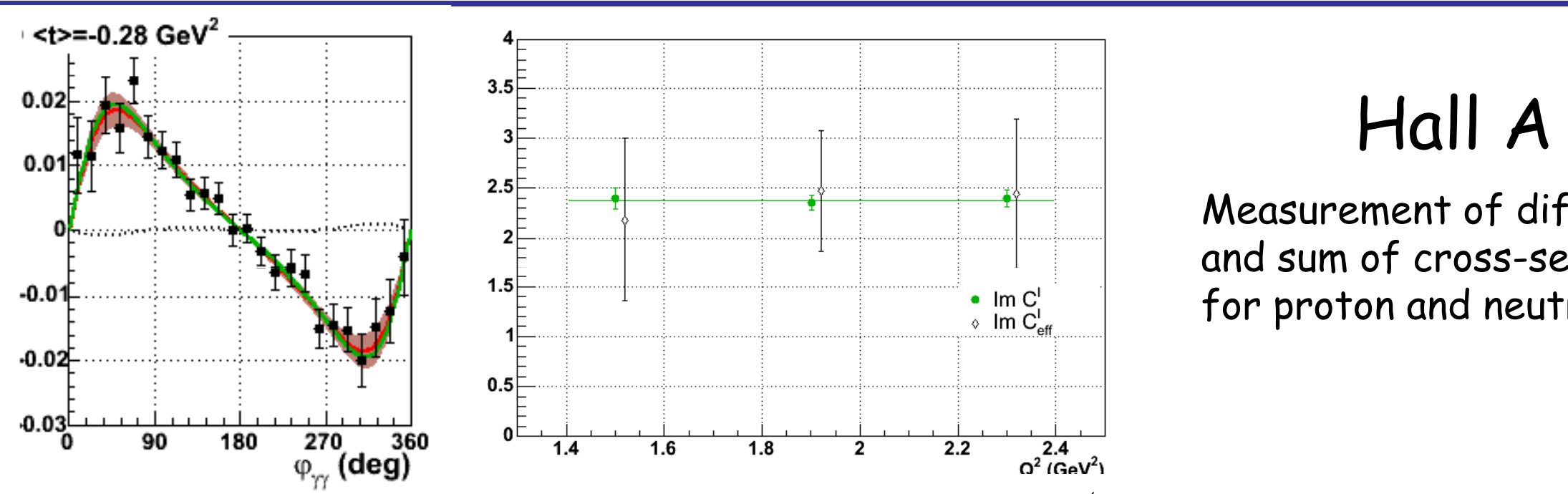

Measurement of difference and sum of cross-sections for proton and neutron

$C^{I}(F)=F_{1} \mathrm{H}+\frac{x_{B}}{2-x_{B}}\left(F_{1}+F_{2}\right) \tilde{\mathrm{H}}-\frac{t}{4 M^{2}} F_{2} \mathrm{E}$
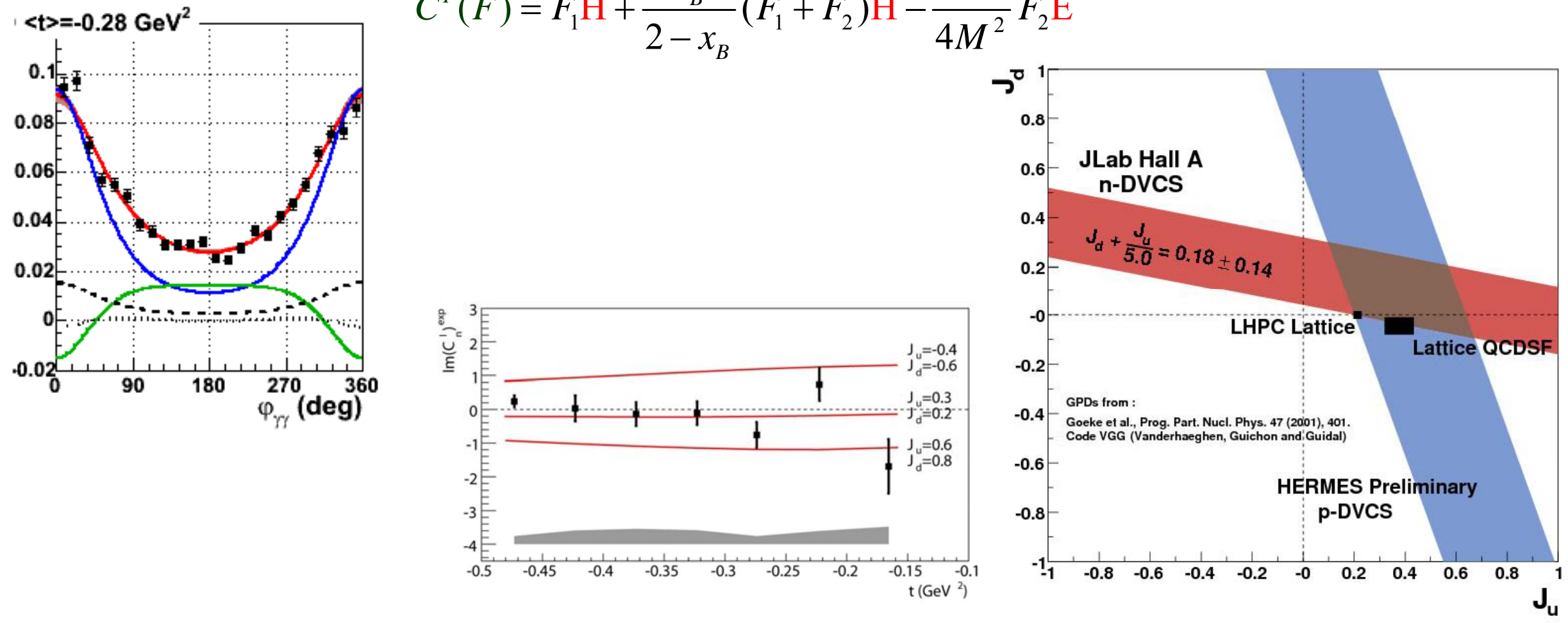


\section{$6 \mathrm{GeV}$ breakthroughs and results in a nutshell}

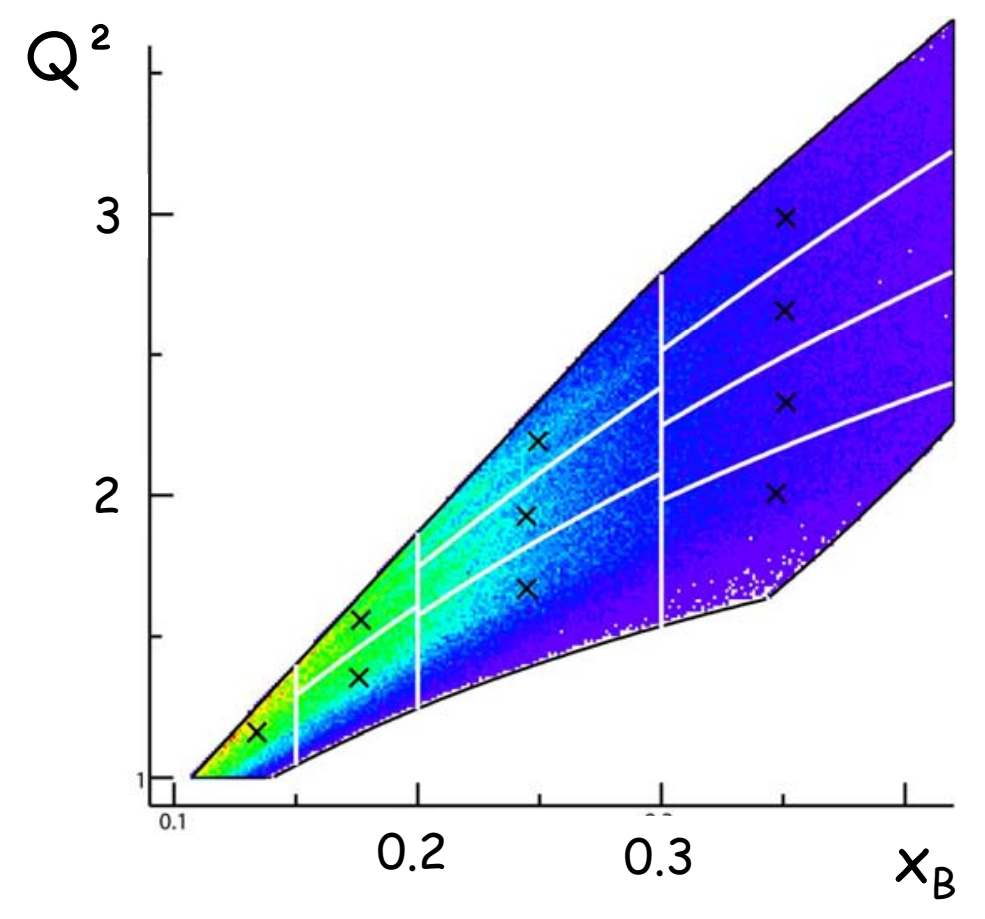

\section{Hall B}

Measurement of $A_{L U}$ and $A_{U L}$ on the proton

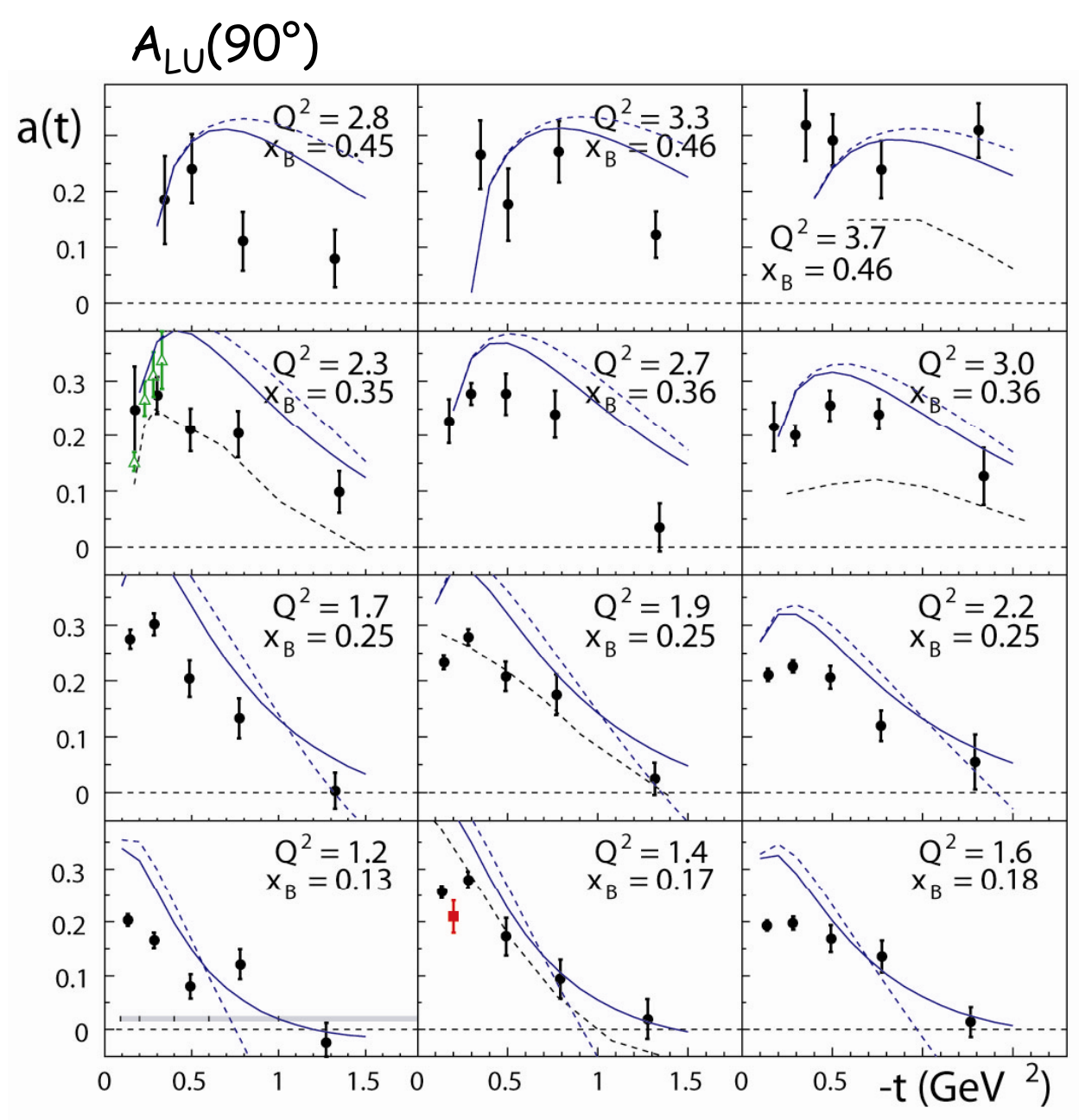




\section{Extension of the Hall A experiments}

- 88 days on $\mathrm{H}$ target (BSA)

- L $\approx 4 \times 10^{37} / \mathrm{cm}^{2} / \mathrm{s} @ 6.6,8.8$ and $11 \mathrm{GeV}$

Exclusivity ensured

by high resolution
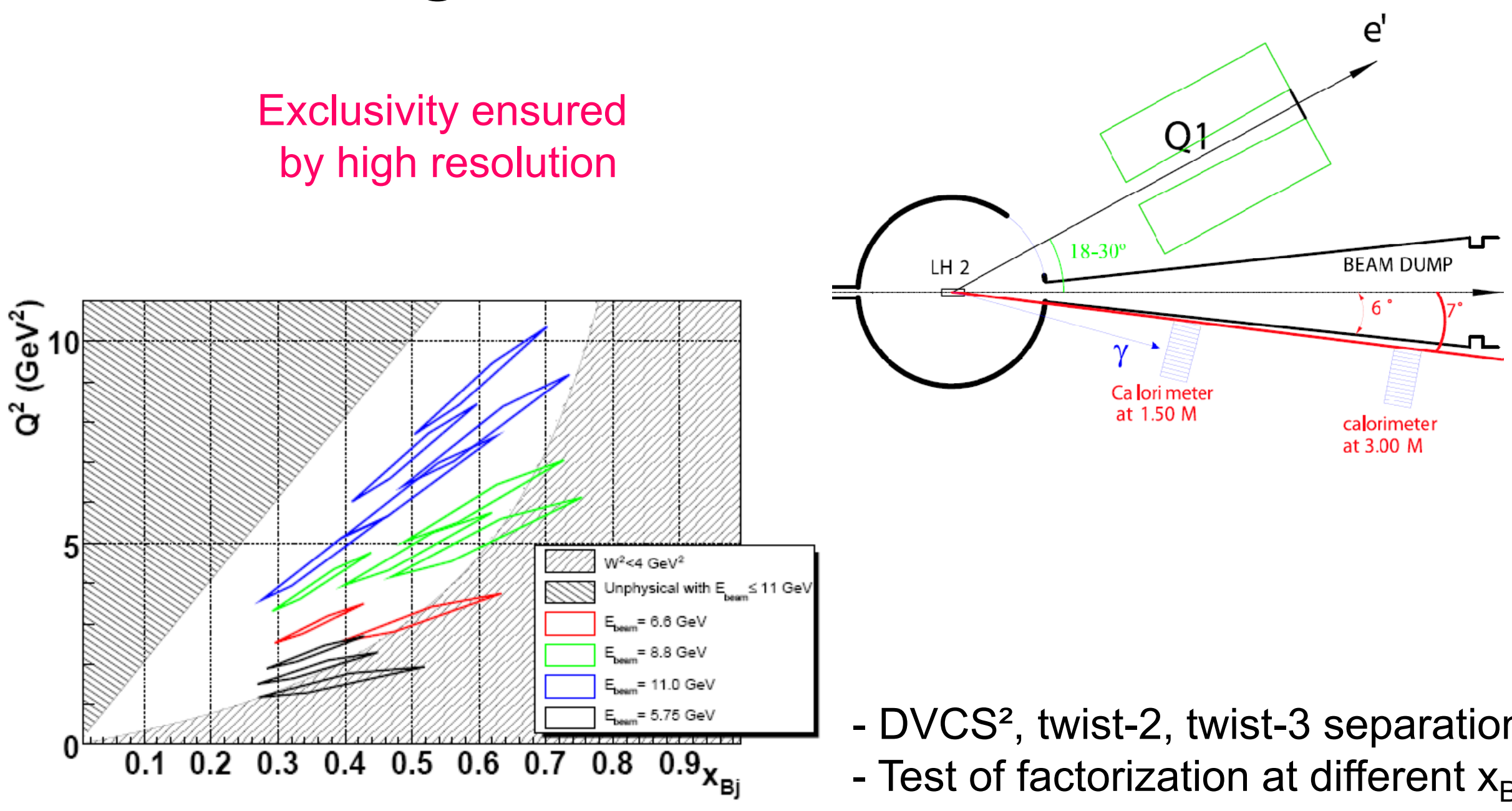

- DVCS ${ }^{2}$, twist-2, twist-3 separation

- Test of factorization at different $x_{B}$ 


\section{Expected accuracy (only a few bins)}
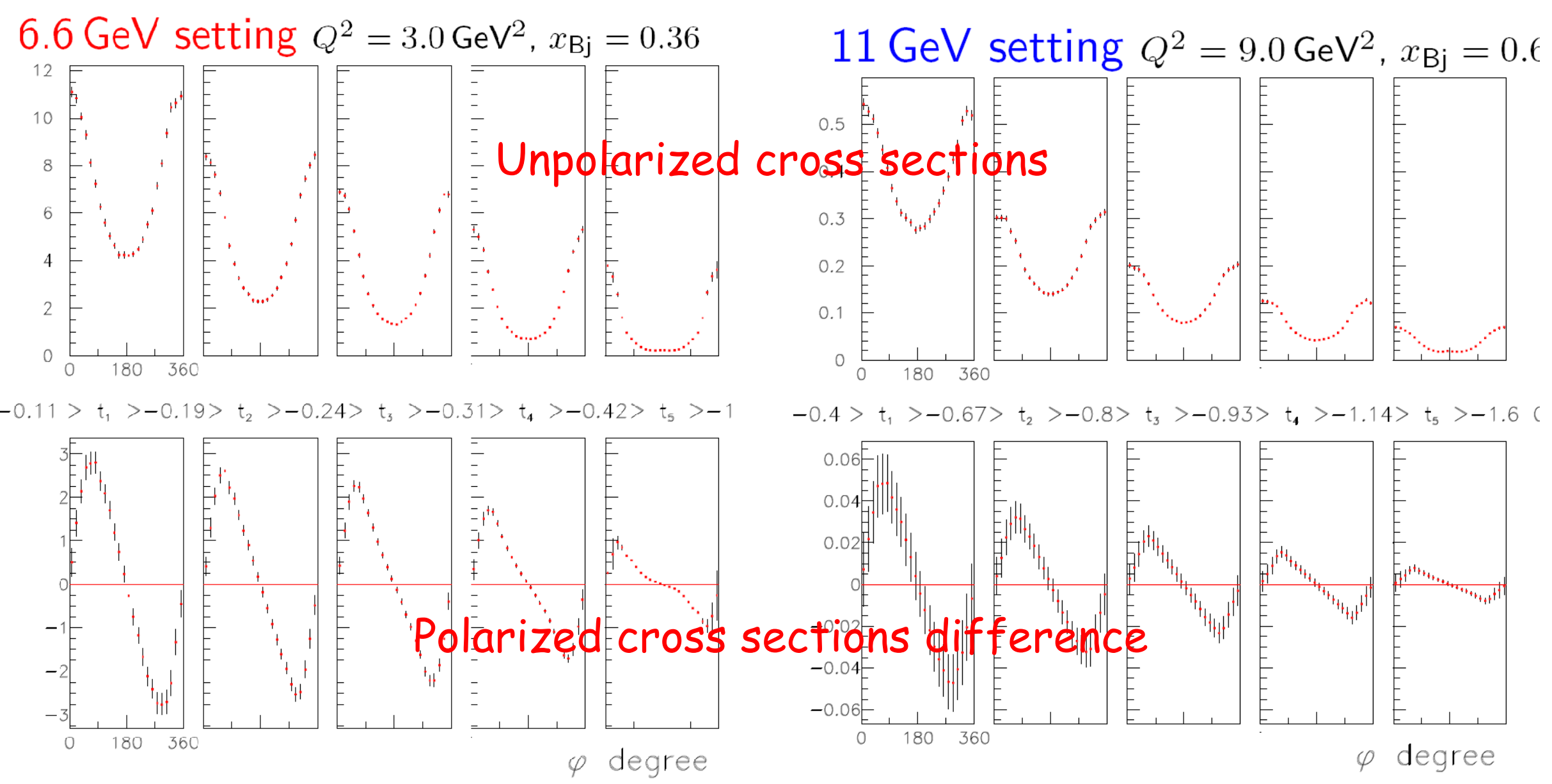

$\rightarrow$ Access the real and imaginary parts of TDVCS separately 


\section{Strategy for the CLAS12 detector in Hall B}

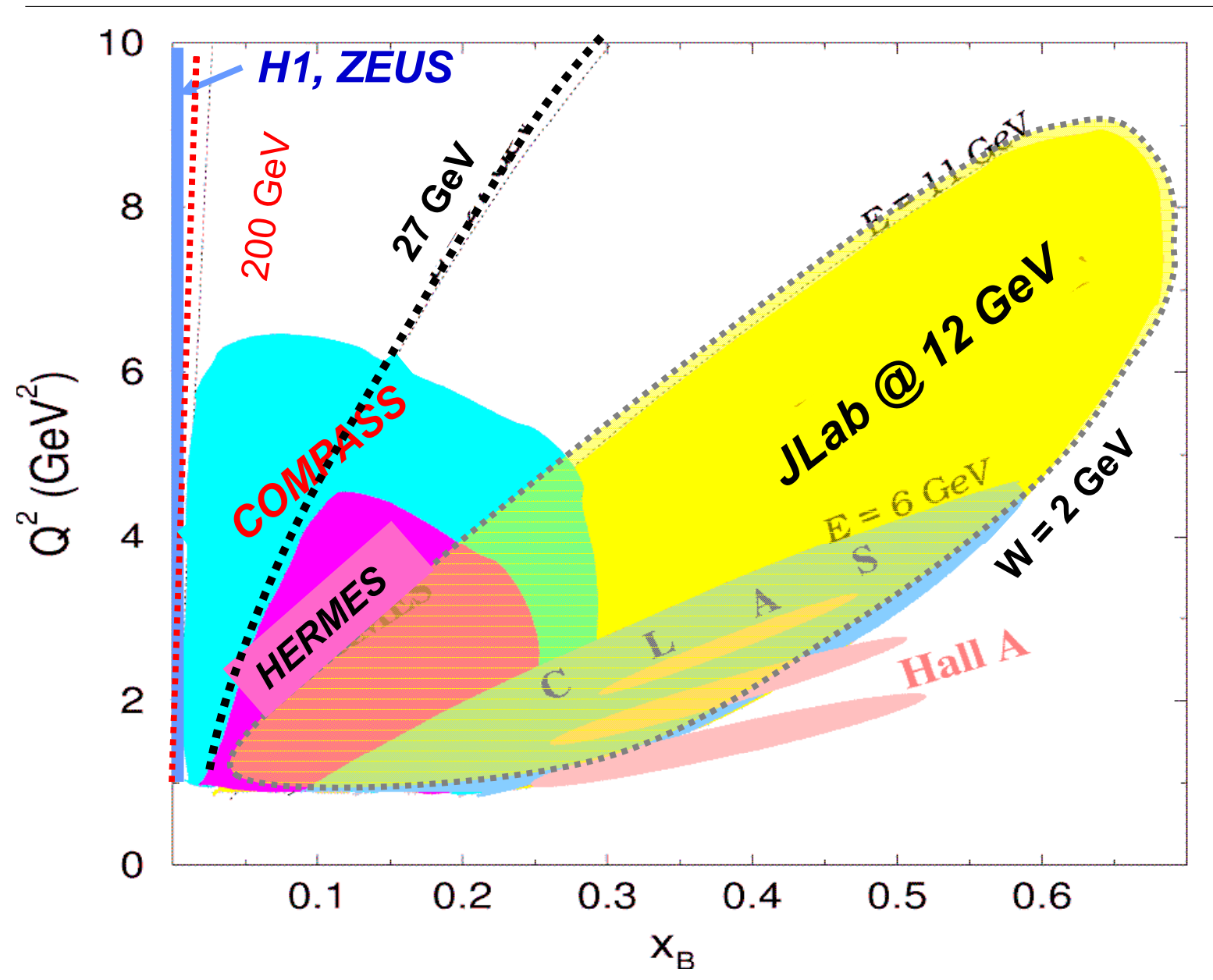

The CLAS12 detector covers a large kinematical domain, especially at high-x 


\section{Measuring DVCS using all polarization settings !}

CLAS12 will have access to beam and target polarizations (both $L$ and $T$ )

With polarized beam and unpolarized target:

$$
\Delta \sigma_{L U} \square \sin \varphi\left\{F_{1} H+\xi\left(F_{1}+F_{2}\right) \tilde{H}+\left(t / 4 M^{2}\right) F_{2} E\right\} d \varphi
$$

With unpolarized beam and Longitudinally polarized target:

$$
\Delta \sigma_{L U} \square \sin \varphi\left\{F_{1} \tilde{H}+\xi\left(F_{1}+F_{2}\right) H+\left(t / 4 M^{2}\right) F_{2} E\right\} d \varphi
$$

With unpolarized beam and Transversely polarized target:

$$
\Delta \sigma_{U T} \square \cos \varphi \sin \left(\phi_{S}-\varphi\right)\left\{\left(t / 4 M^{2}\right) F_{2} H-\left(t / 4 M^{2}\right) F_{1} E+\ldots\right\} d \varphi
$$

Depending on the experiment, measurement of $\Delta \sigma$ and $\sigma$ or $A=\frac{\Delta \sigma}{2 \sigma}$ 
Expected accuracy for $\sin \varphi$ term in $A_{L U}$ as a function of $\dagger$
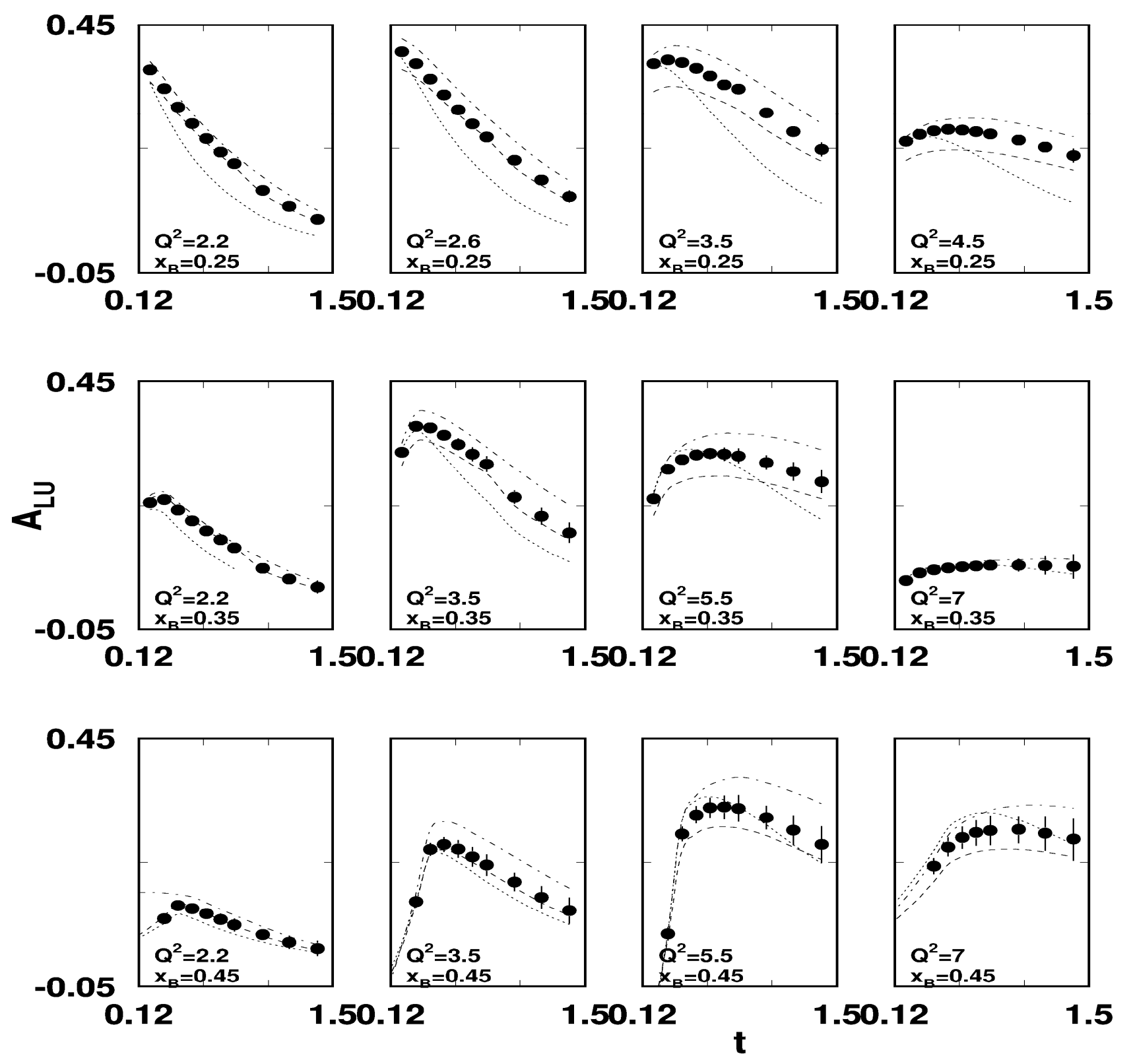
Extraction of the GPD $H(x=\xi)$ from projected CLAS12 data

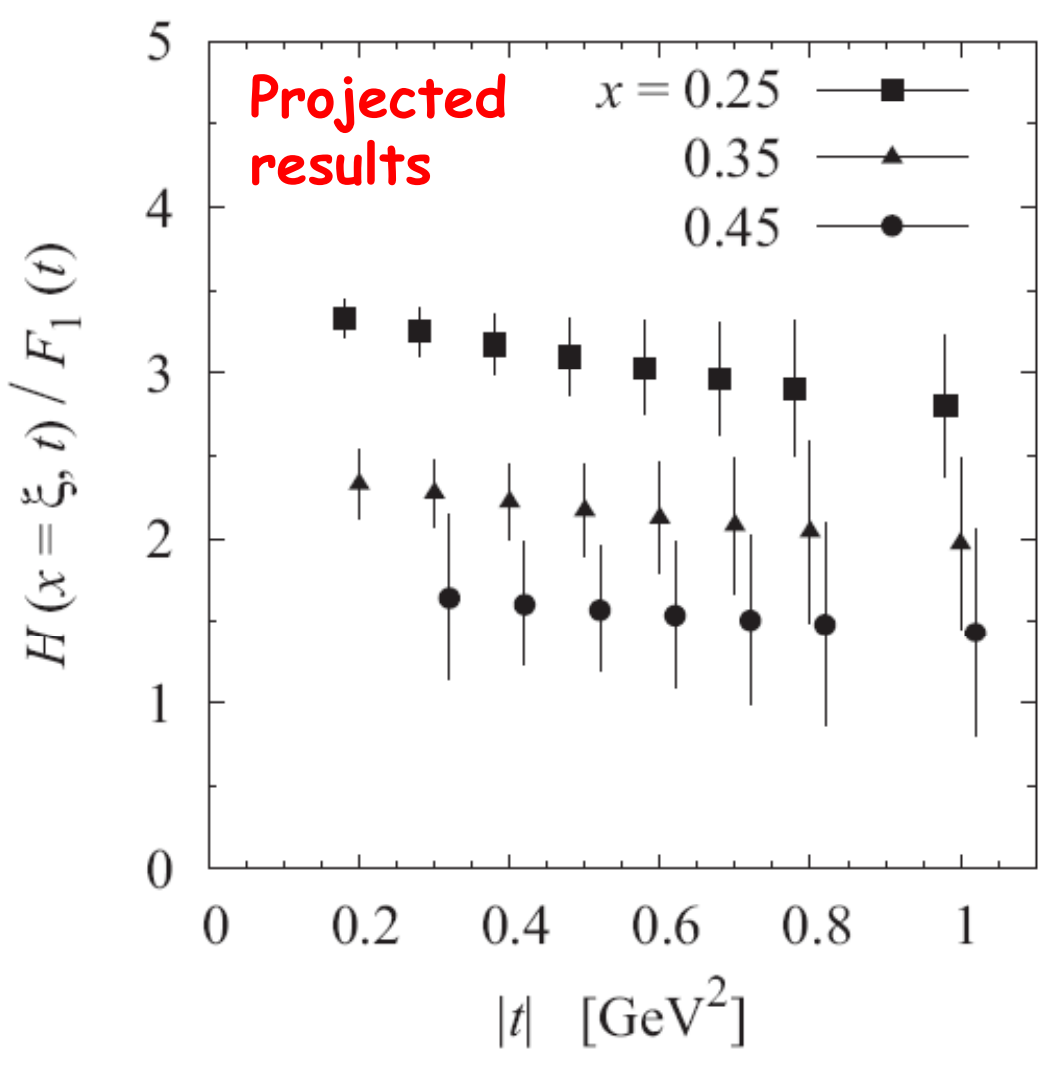

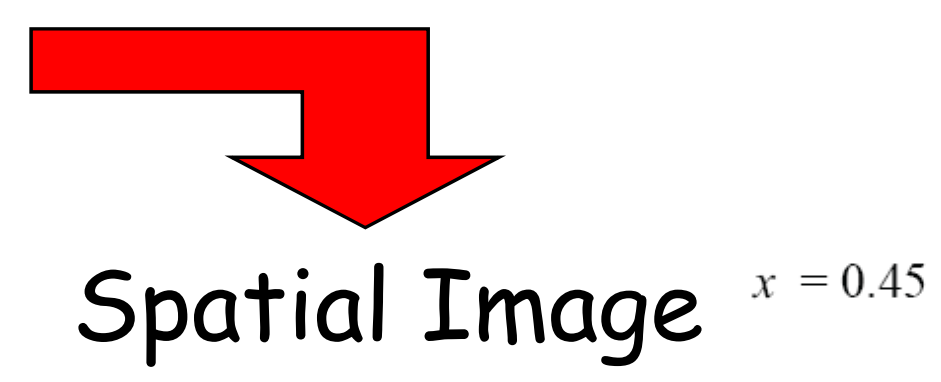

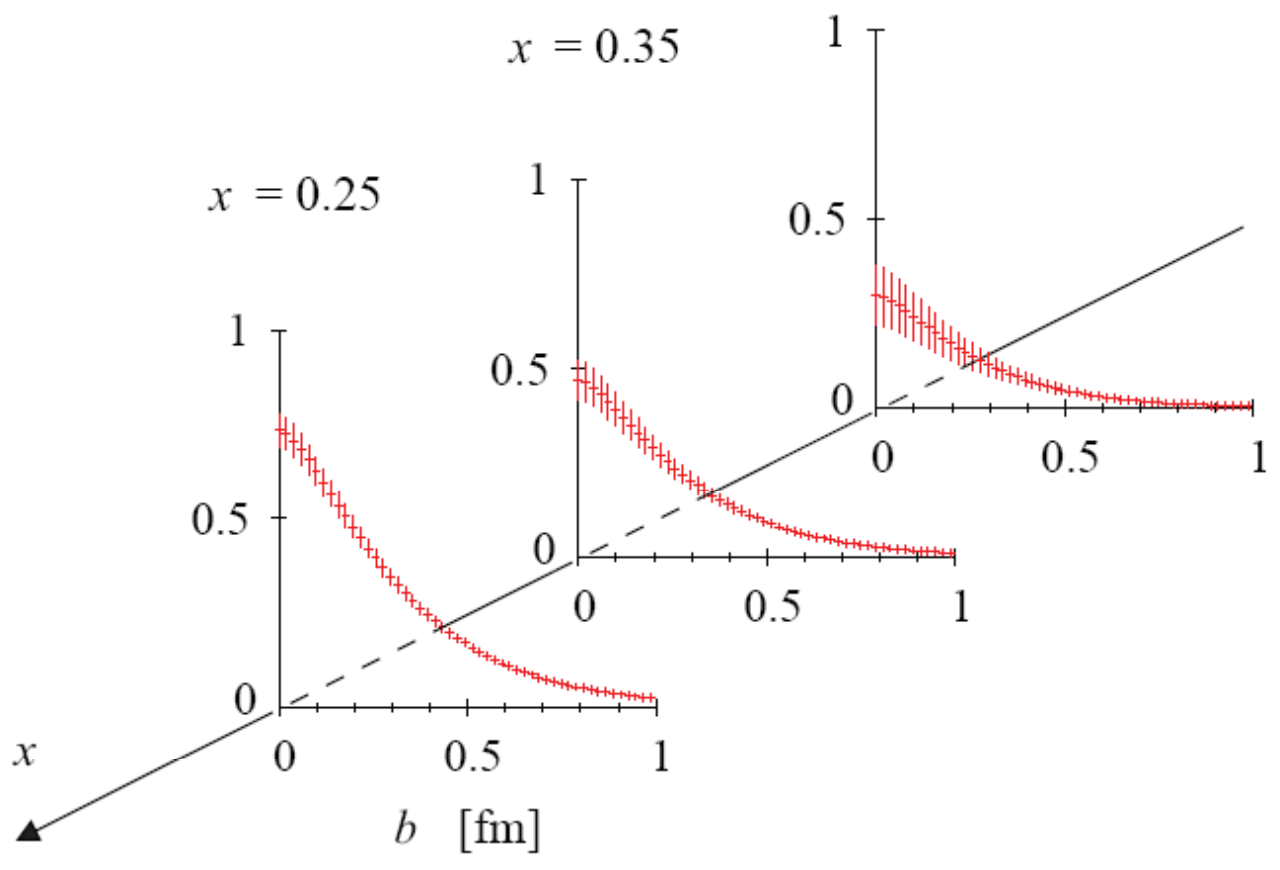




\section{Summary}

- The JLab 12 GeV Upgrade has well defined physics goals of fundamental importance for the future of hadron physics, addressing in new and revolutionary ways the quark and gluon structure of mesons, nucleons, and nuclei by

- accessing Generalized Parton Distributions

- exploring the valence quark structure of the nucleon

- understanding quark confinement and hadronization processes

- extending nucleon elastic and transition Form Factors to short distances

- mapping the spectrum of gluonic excitations of mesons

- searching for physics beyond the Standard Model through parity violation

- Design of accelerator and equipment upgrades are underway

- Construction has begun!

- Accelerator shutdown scheduled for 2012

- Upgrade completion scheduled in 2015 


\section{More information on JLab 6 and 12 physics in this conference}

by order of appearance...

(I may have forgotten a few !)

Nucleon Valence Structure (SIDIS, High X, etc): C. Keppel, V. Burkert, H. Gao, G. Cates, A. Puckett, X. Zhan, Z-E. Meziani, J-P. Chen

GPD related: P. Nadel-Turowski, M. Burkardt, D. Hamilton, V. Guzey

Spectroscopy and Exotics: M. Shepherd, D. Lawrence

Nuclei: A. Daniel, M. Olson, P. Monaghan, R. Schiavilla, M. Iodice

PV with electrons: P. King, A. Thomas, P. Souder, E. Beise

In sessions:

Nuclear and Nucleon Structure 1 to 9

Plenary 7

Hadronic and Spin Physics 4

Hadron Spectroscopy and Exotics 5

New Facilities and Instrumentation 4 


\section{BACKUP}




\section{Published non-dedicated results on $A_{L U}$ and $A_{U L}$}

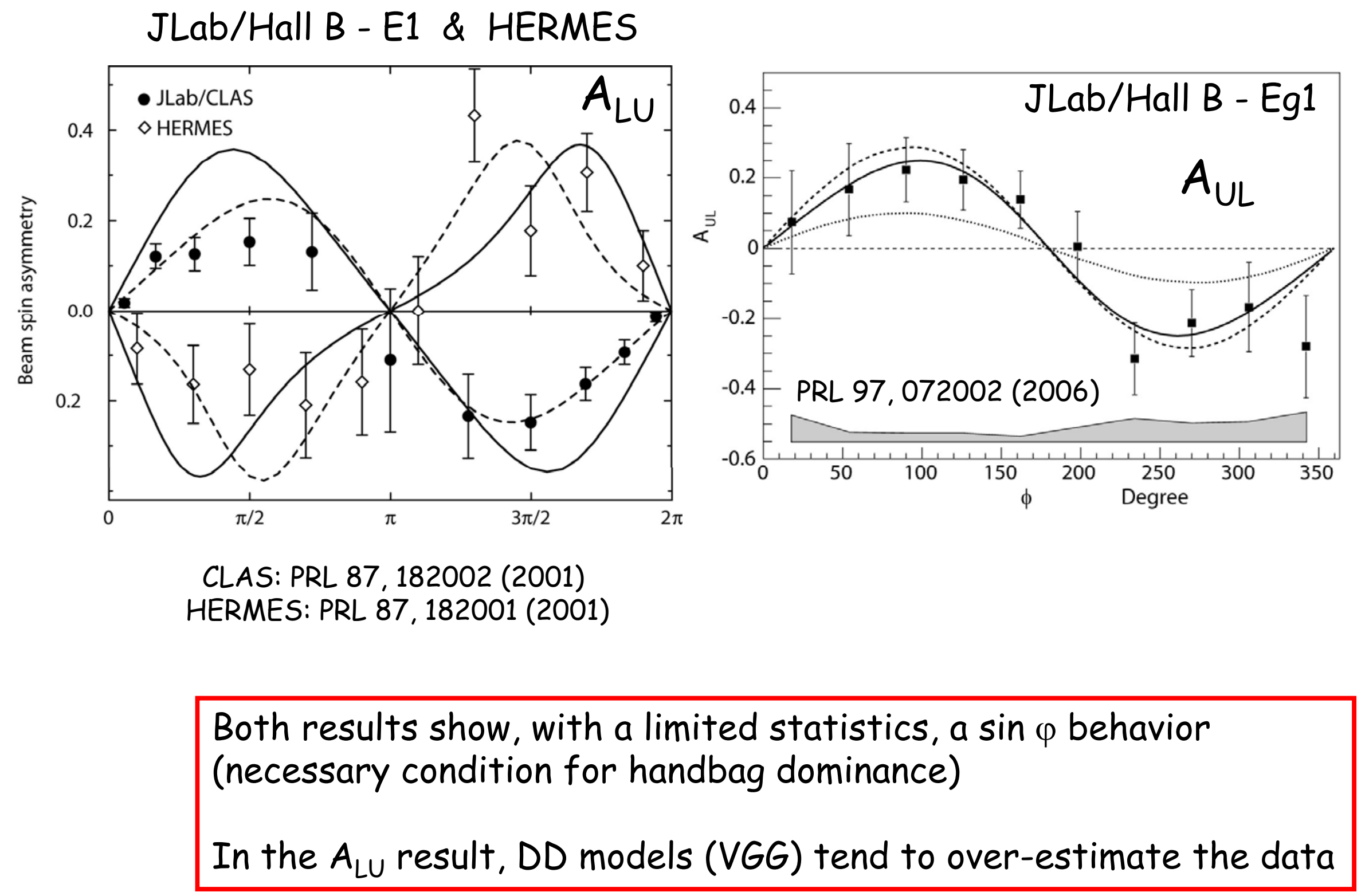




\section{E00-110 experimental setup}

- $75 \%$ polarized $2.54 A$ electron beam

- $15 \mathrm{~cm}$ LH2 target

- Left Hall A HRS with electron package

- 11x12 block PbF2 electromagnetic calorimeter

- 5x20 block plastic scintillator array
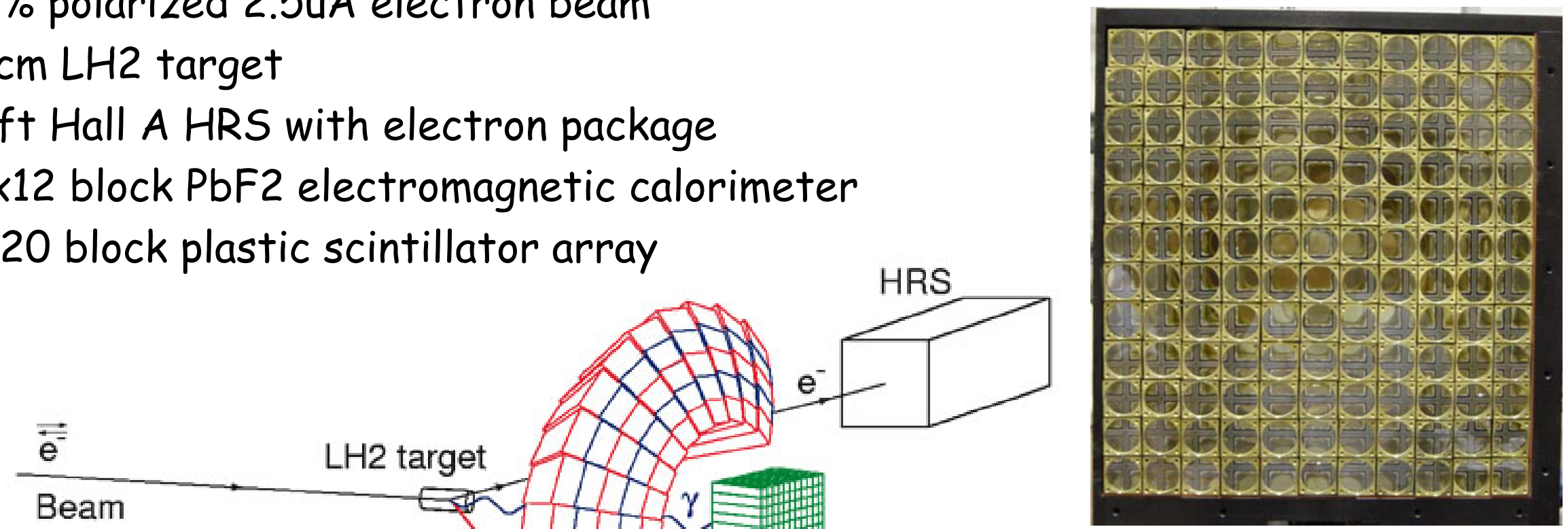

50 days of beam time in the fall 2004, at $2.5 \mu \mathrm{A}$

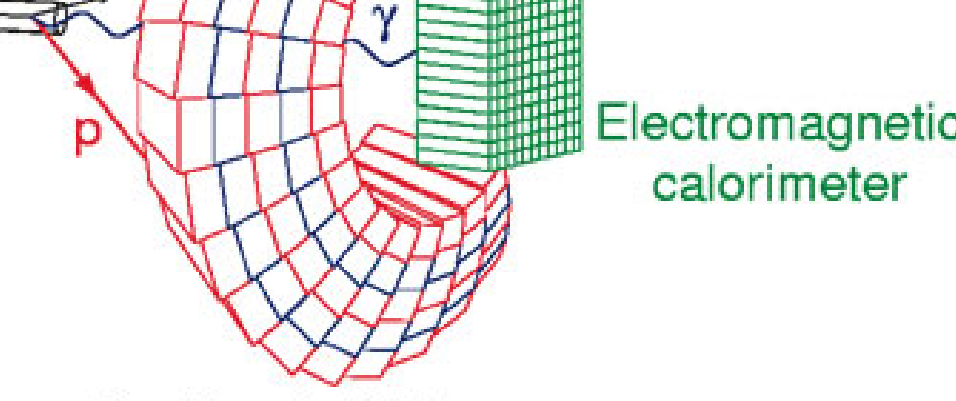

Plastic scintillator array

$\int L u \cdot d t=13294 \mathrm{fb}^{-1}$

\begin{tabular}{|c|c|c|c|c|}
\hline Kin & $\begin{array}{c}Q^{2} \\
\left(\mathrm{GeV}^{2}\right)\end{array}$ & $x_{B}$ & $\begin{array}{c}\theta_{\gamma^{*}} \\
(\text { deg. })\end{array}$ & $\begin{array}{c}W \\
(\mathrm{GeV})\end{array}$ \\
\hline \hline 1 & $\mathbf{1 . 5}$ & 0.36 & $\mathbf{2 2 . 3}$ & 1.9 \\
\hline 2 & $\mathbf{1 . 9}$ & 0.36 & $\mathbf{1 8 . 3}$ & 2.0 \\
\hline 3 & $\mathbf{2 . 3}$ & 0.36 & $\mathbf{1 4 . 8}$ & 2.2 \\
\hline
\end{tabular}

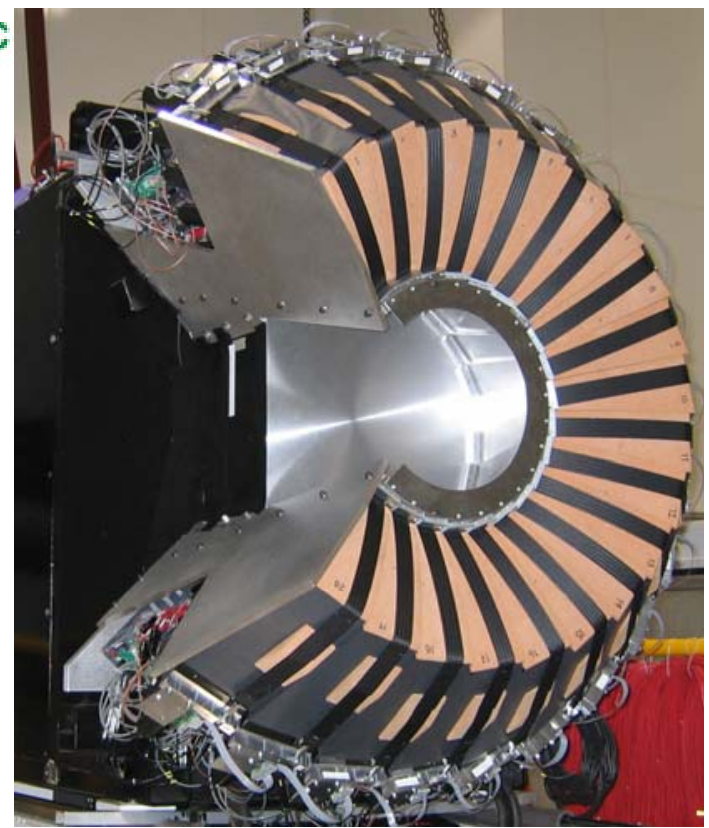




\section{Difference of cross-sections}

PRL97, 262002 (2006)

$$
\begin{aligned}
& \left\langle Q^{2}\right\rangle=2.3 \mathrm{GeV}^{2} \\
& \left\langle x_{B}\right\rangle=0.36
\end{aligned}
$$

$\frac{1}{2}\left(\frac{d^{4} \sigma^{+}}{d Q^{2} d x_{B} d t d \varphi_{Y Y}}-\frac{d^{4} \sigma^{*}}{d Q^{2} d x_{B} d t d \varphi_{Y Y}}\right)\left(n b / G e V^{4}\right)$
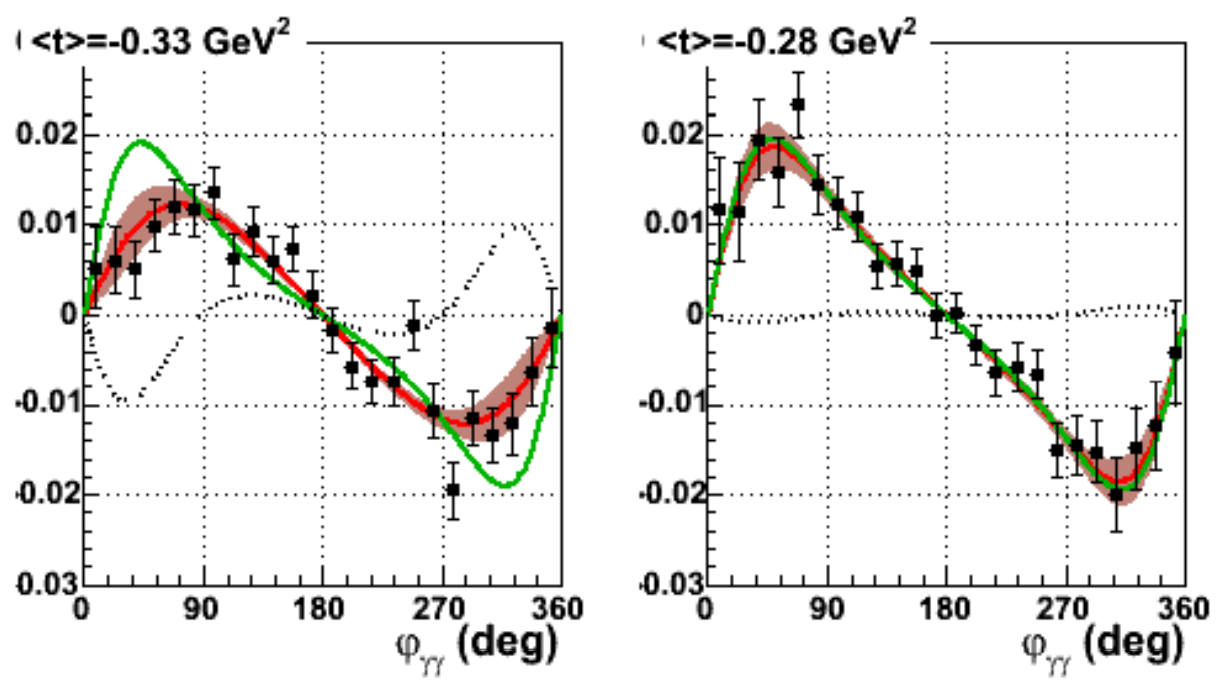

Corrected for real+virtual RC Corrected for efficiency Corrected for acceptance Corrected for resolution effects Checked elastic cross-section @ 1\%
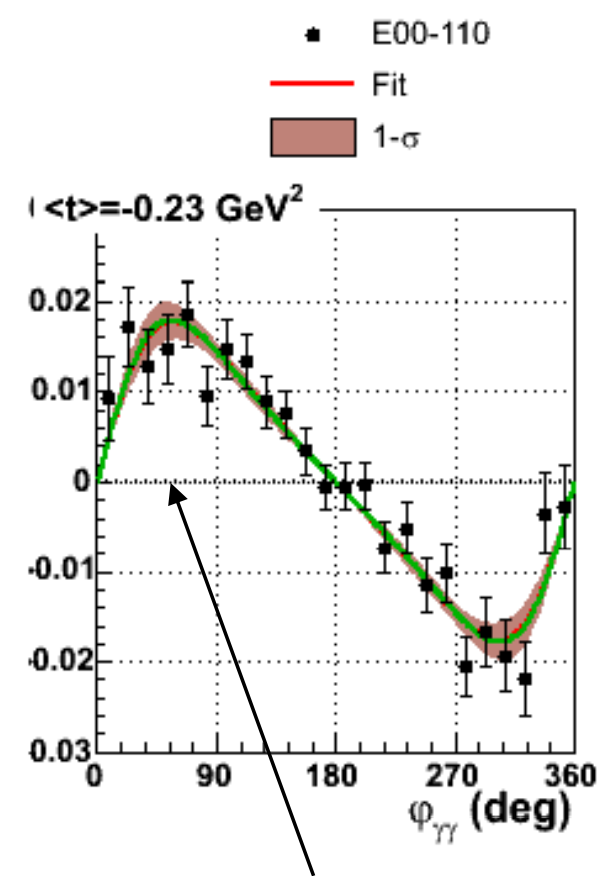

Extracted Twist-3 contribution small!
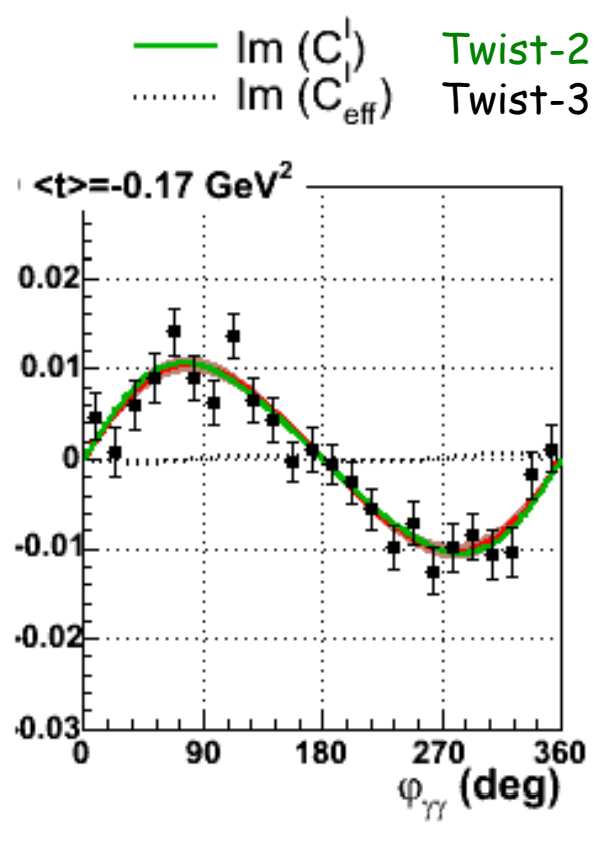


\section{$Q^{2}$ dependence and test of scaling}

PRL97, 262002 (2006)

$$
\langle-t\rangle=0.26 \mathrm{GeV}^{2},\left\langle x_{B}\right\rangle=0.36
$$

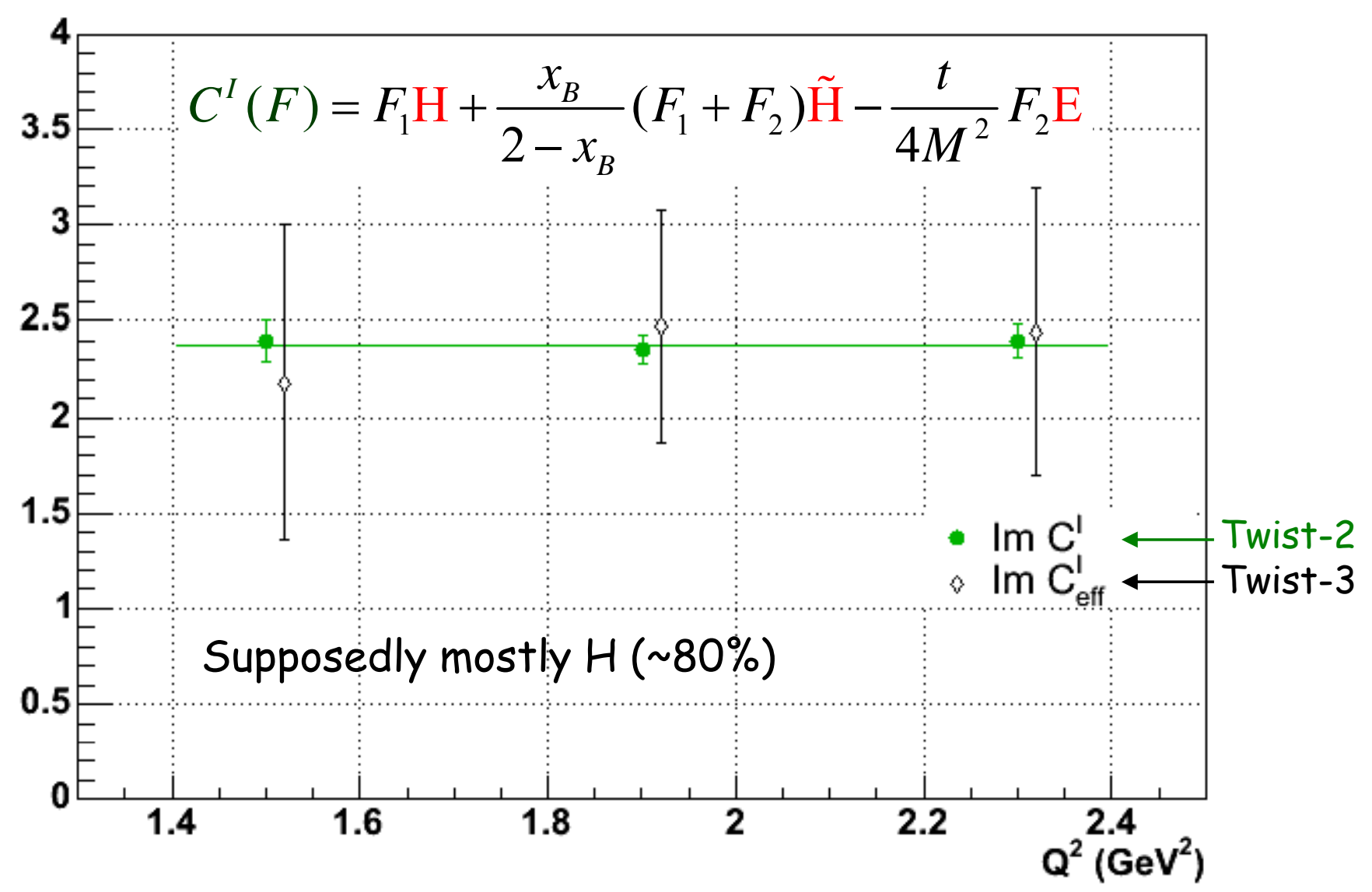

No $Q^{2}$ dependence using BMK separation: strong indication for scaling behavior and handbag dominance

Twist 4+ contributions are smaller than $10 \%$ 


\section{Total cross-section}

PRL97, 262002 (2006)

$$
\frac{d^{4} \sigma}{d Q^{2} d x_{B} d t d \varphi_{Y Y}}\left(n b / G e V^{4}\right)
$$
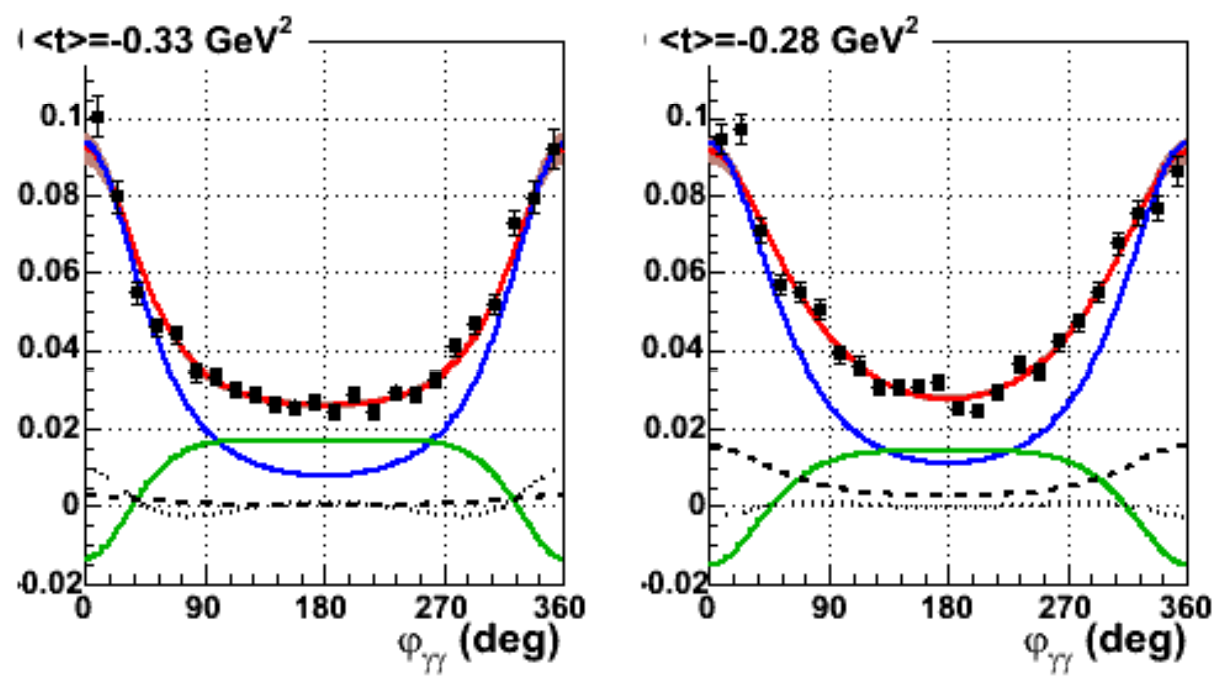

Corrected for real+virtual RC Corrected for efficiency Corrected for acceptance Corrected for resolution effects

$$
\begin{aligned}
& \left\langle Q^{2}\right\rangle=2.3 \mathrm{GeV}^{2} \\
& \left\langle x_{B}\right\rangle=0.36
\end{aligned}
$$

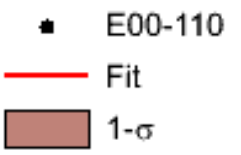

$1<t>=-0.23 \mathrm{GeV}^{2}$

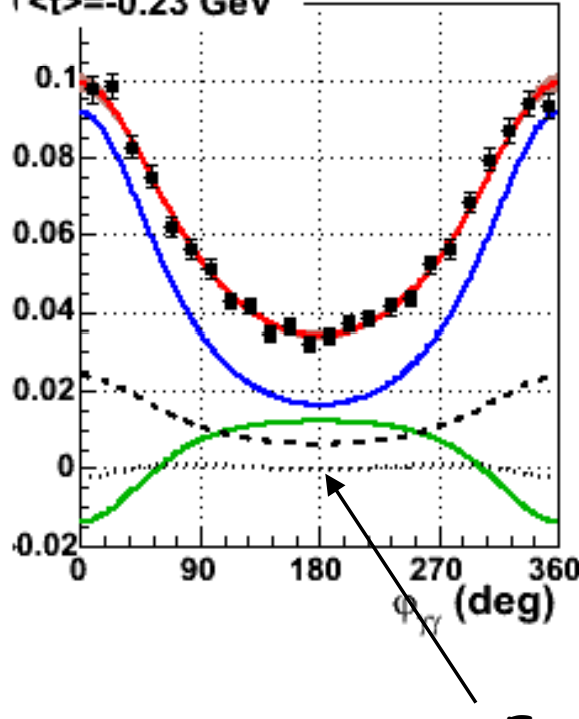

Extracted Twist-3 contribution small!

but impossible to disentangle DVCS 2 from the interference term! 


\section{DVCS on the neutron in JLab/Hall A: E03-106}

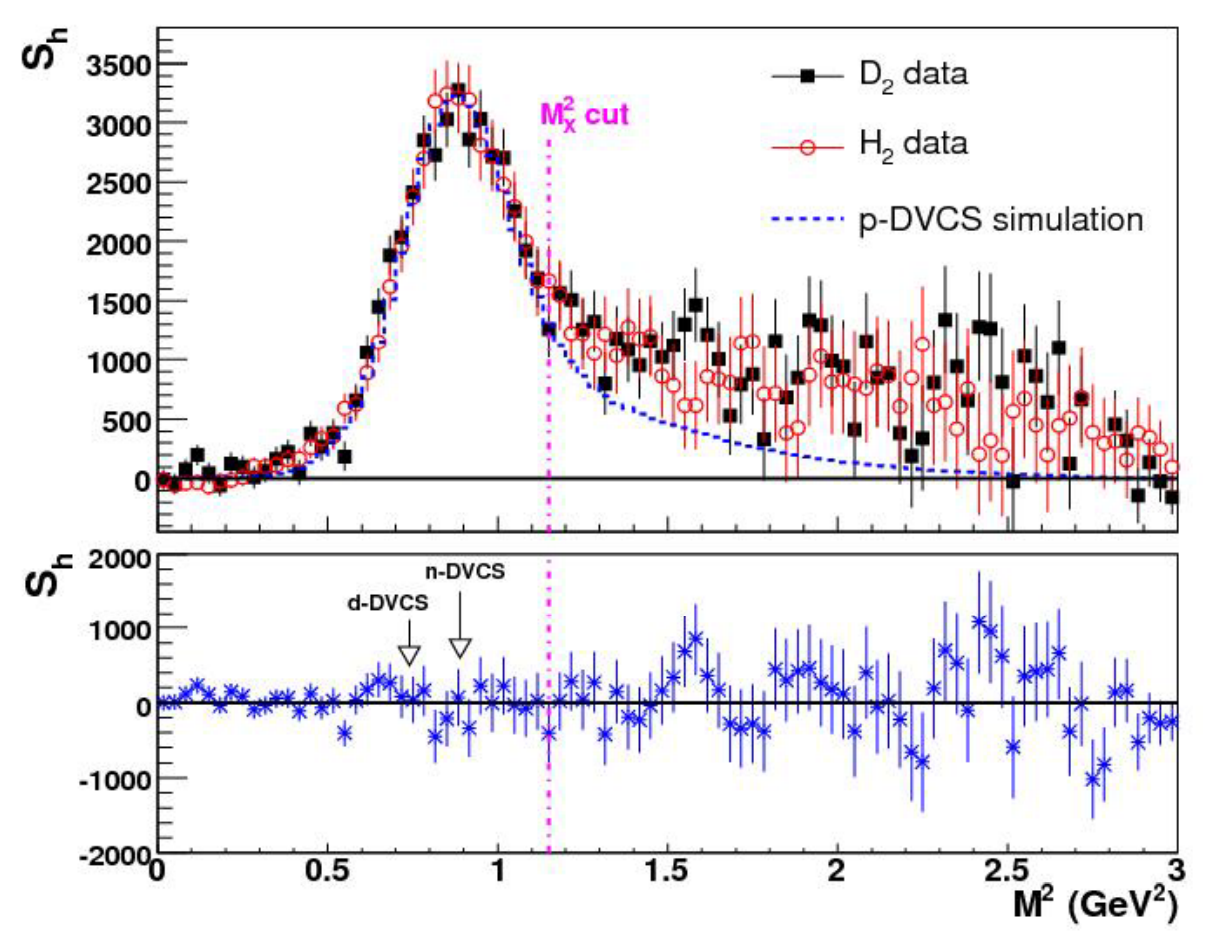

$L D_{2}$ target
$24000 \mathrm{fb}-1$
$x_{B}=0.36, Q^{2}=1.9 \mathrm{GeV}^{2}$

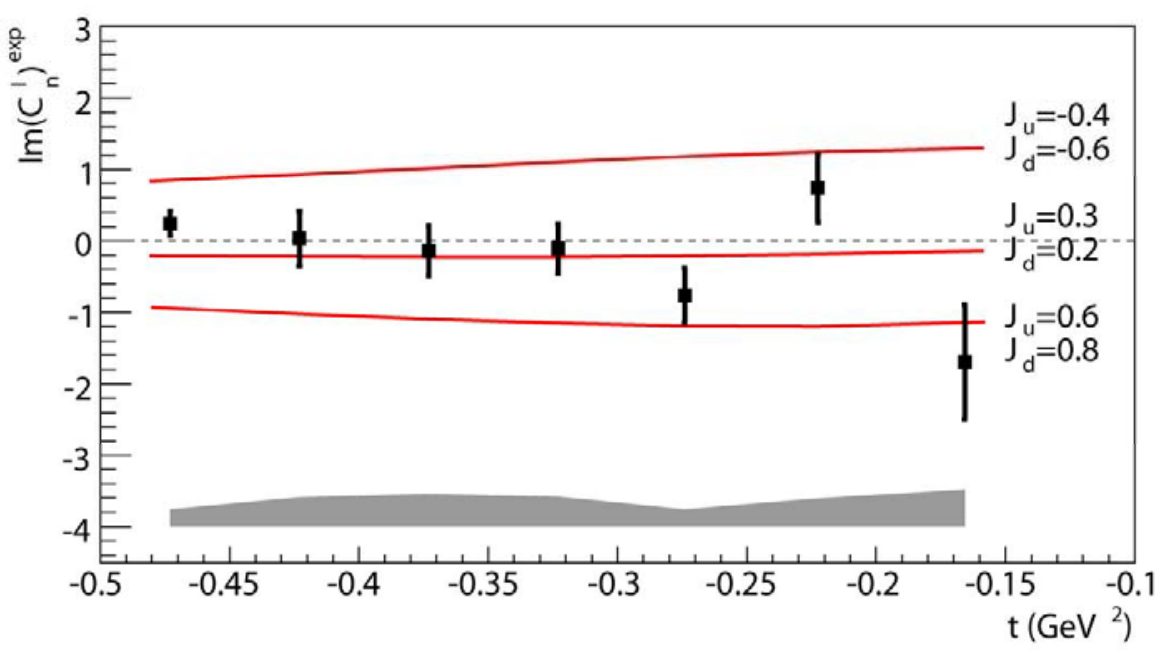

\section{MODEL-DEPENDENT}

Ju-Jd extraction

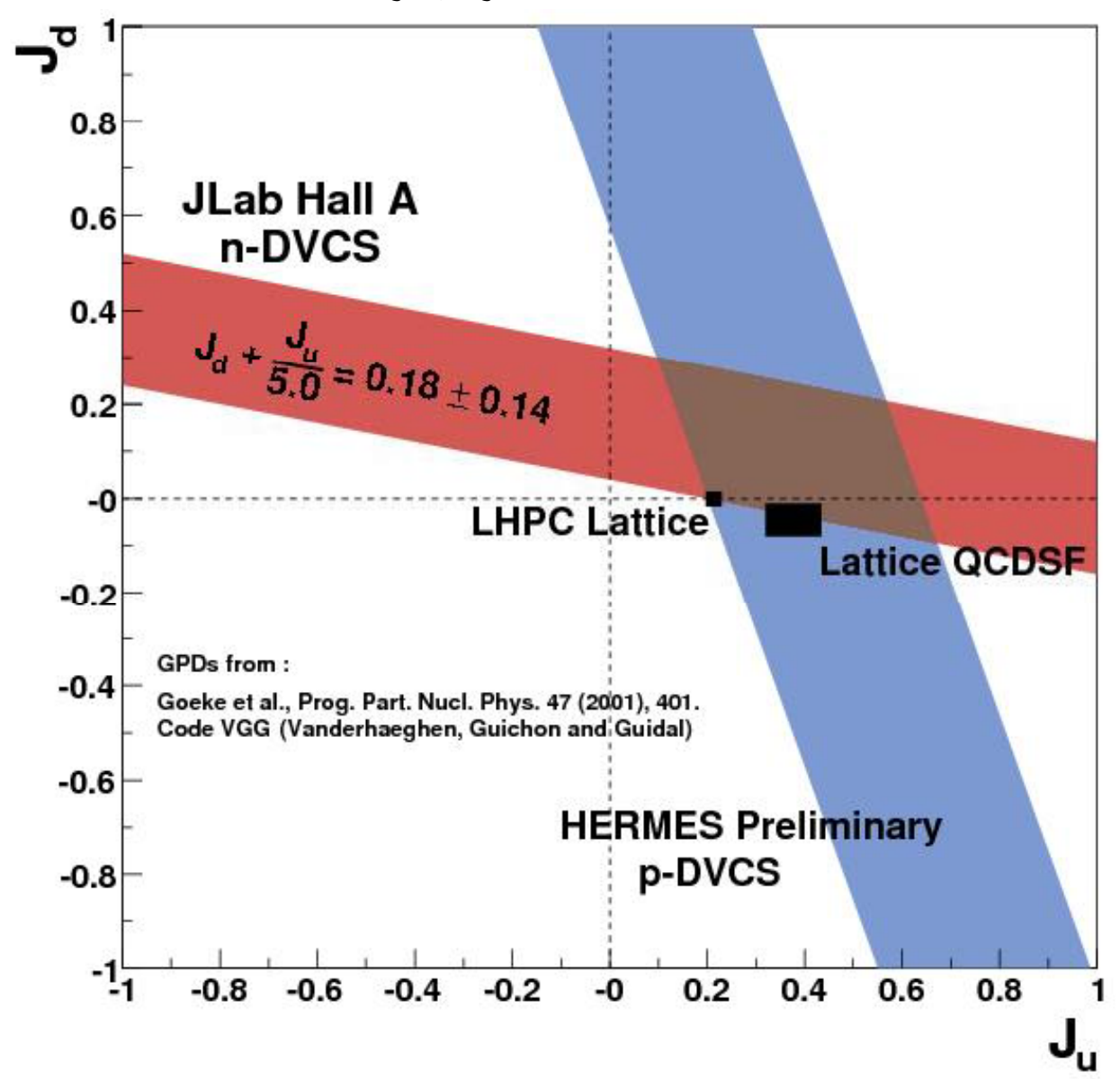




\section{E1-DVCS with CLAS : a dedicated DVCS experiment in Hall B}

$\begin{array}{ll}\text { Beam energy: } & \sim 5.8 \mathrm{GeV} \\ \text { Beam Polarization: } & 75-85 \% \\ \text { Integ. Luminosity: } & 45 \mathrm{fb}^{-1}\end{array}$

2nd half of data under analysis
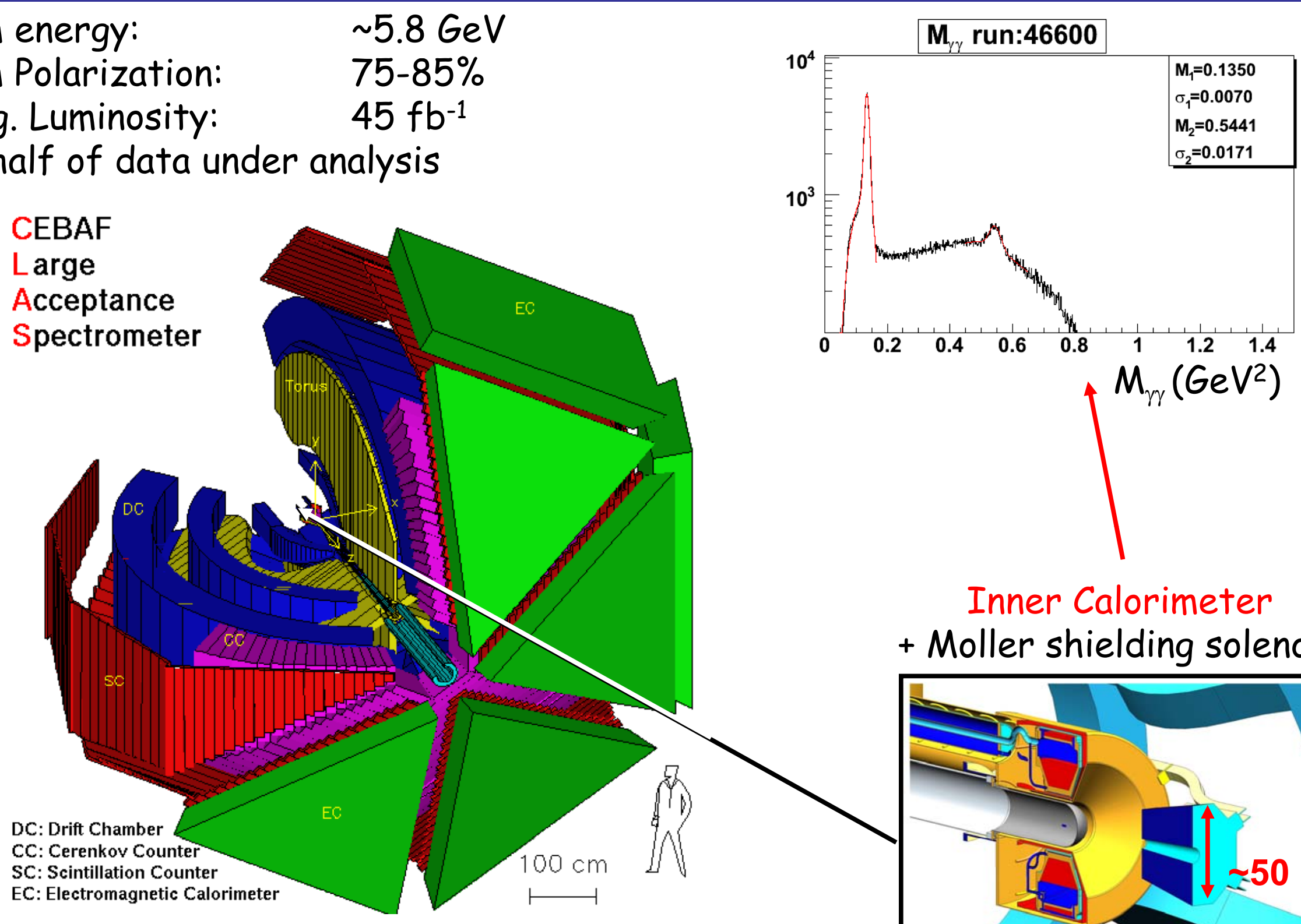

Inner Calorimeter + Moller shielding solenoid

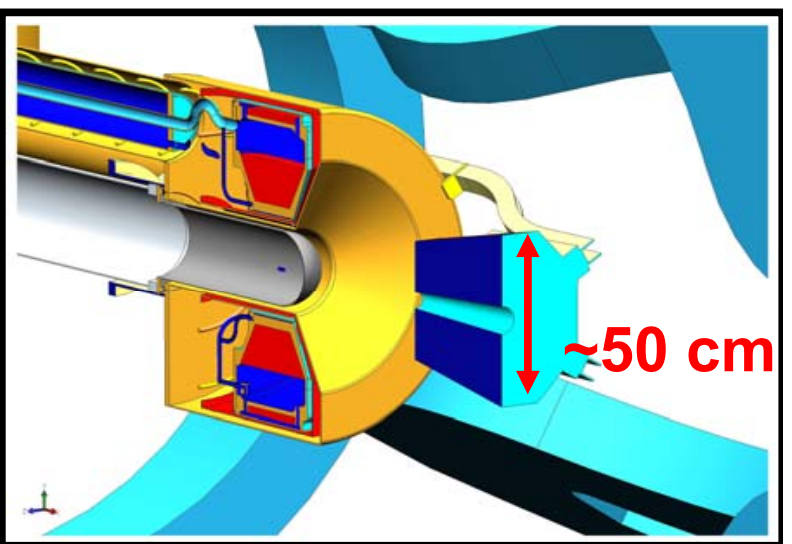




\section{E1-DVCS kinematical coverage and binning}

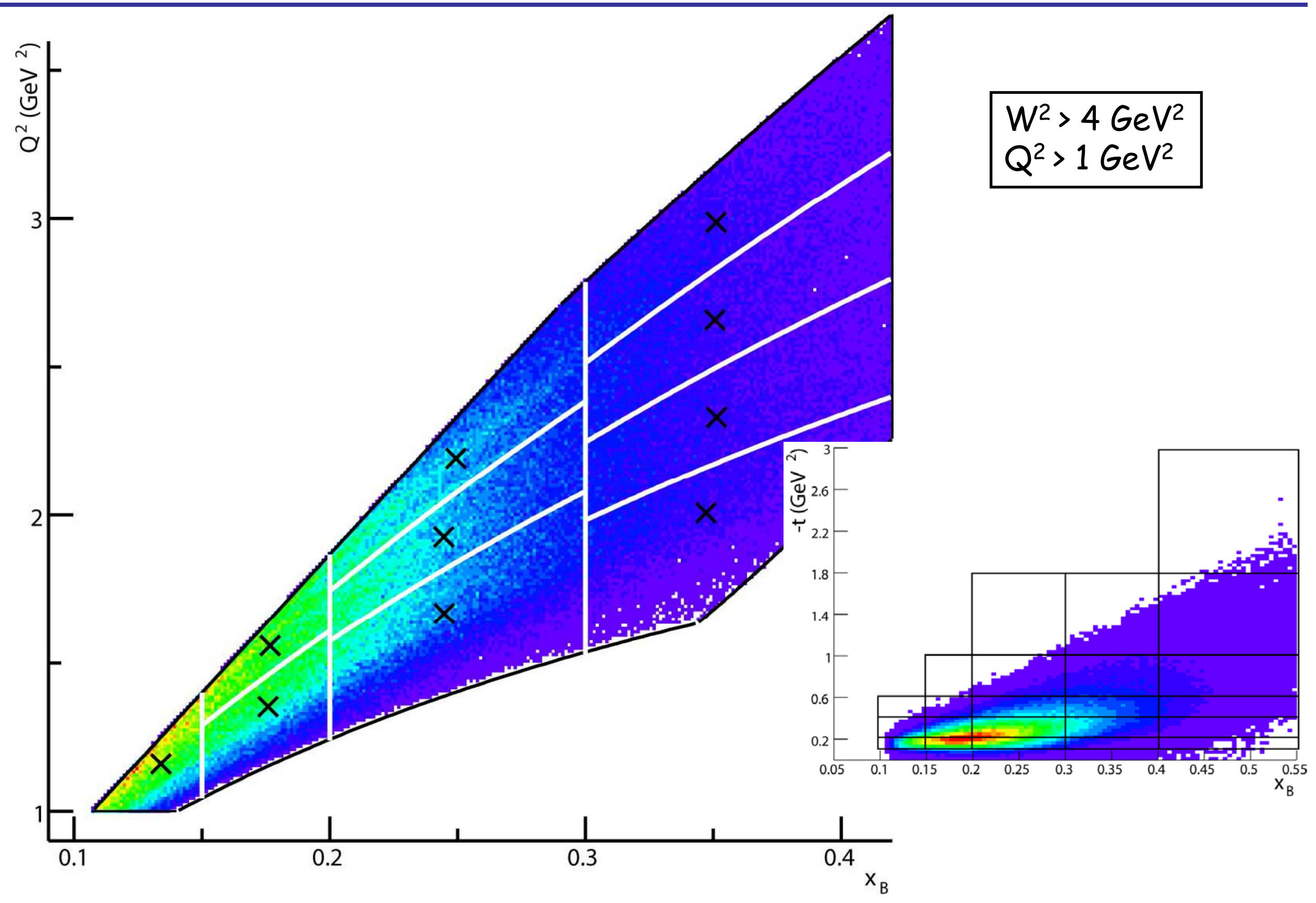




\section{E1-DVCS : Asymmetry as a function of $x_{B}$ and $Q^{2}$}

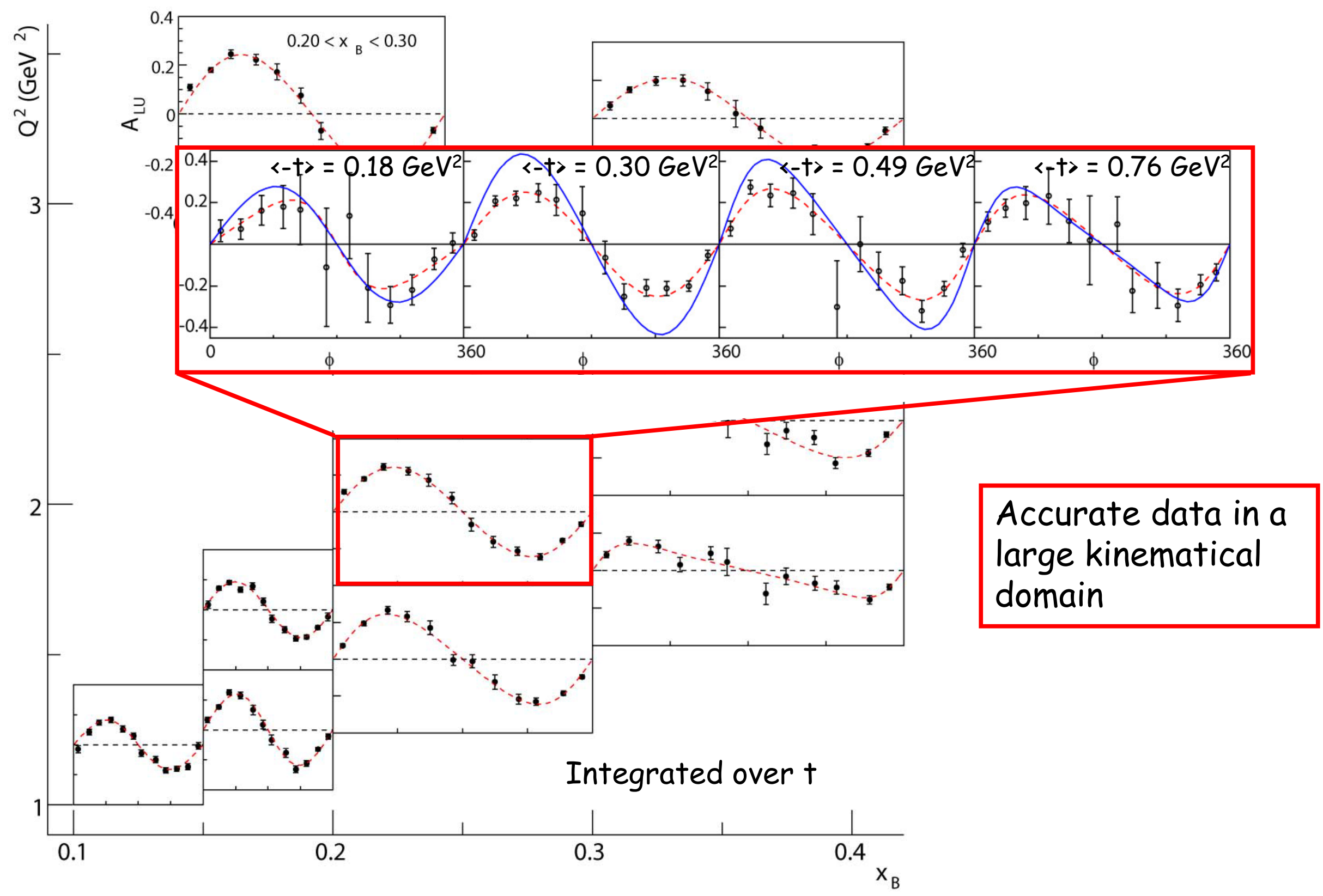




\section{E1-DVCS : $A_{L U}\left(90^{\circ}\right)$ as a function of $-t+$ models}

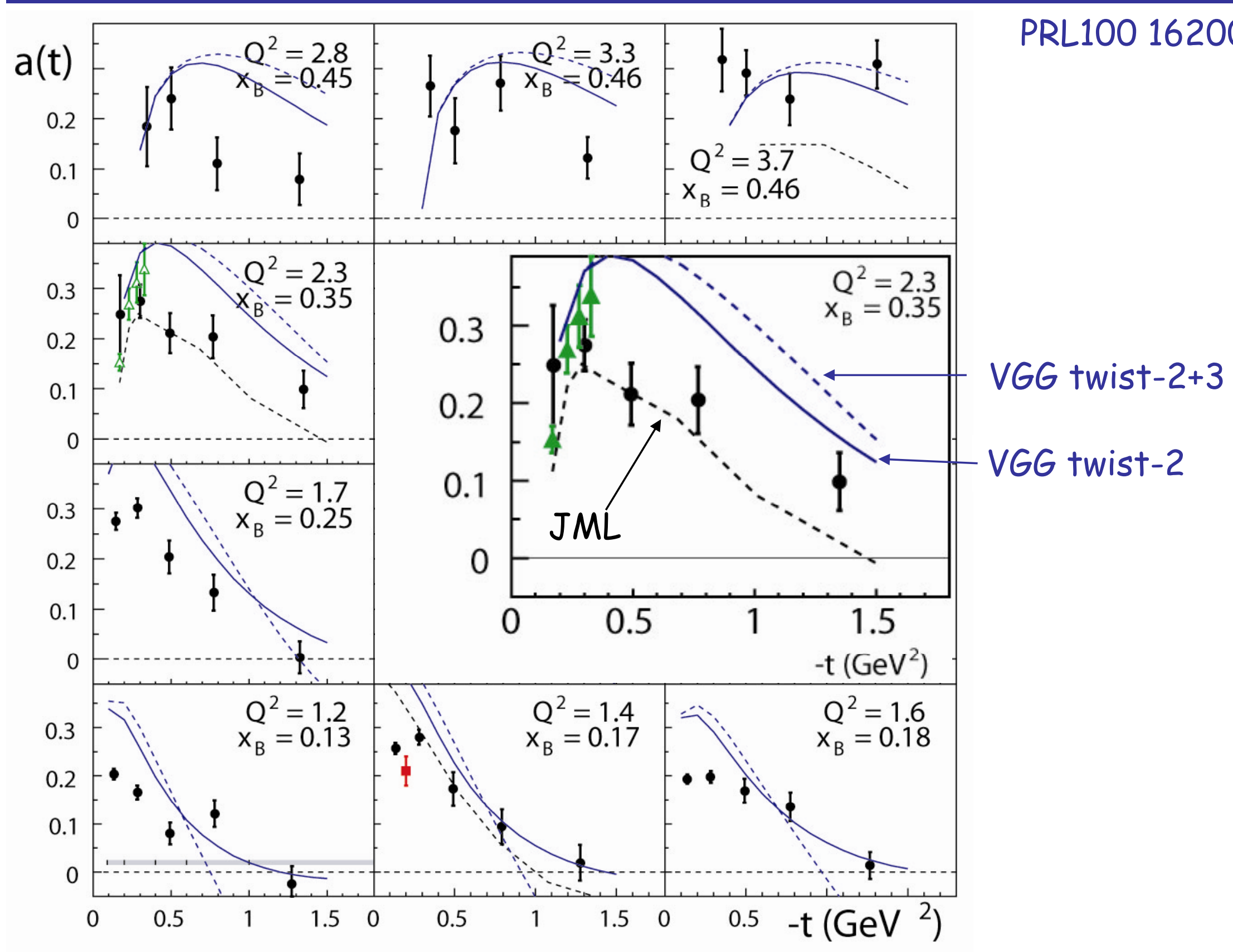




\section{New developments in GPD modeling : minimal dual model}

arXiv:0803.1271v2 Polyakov, Vanderhaeghen

Central quantities are t-dependent parton distributions $q$ and $e(x, t)$, obtained as $\xi \rightarrow 0$ limits of the nucleon GPDs:

$$
q(x, t)=\lim _{\xi \rightarrow 0} H(x, \xi, t), \quad e(x, t)=\lim _{\xi \rightarrow 0} E(x, \xi, t)
$$

$q$ and e are estimated using a dual representation (parton distributions as an infinite series of t-channel exchanges).

Only the leading function is kept for simplicity. Only the GPD H is kept, supposedly dominant in DVCS.

$\longrightarrow$ It is a simple one-parameter model with strong predictive content. If part of the data cannot be fit, it will mean higher twists/genuine nonforward effects need to be included: first step to understand DVCS data. 


\section{Minimal dual model (i.e. forward model) of DVCS data}

Difference of cross sections (imaginary part of interference term)

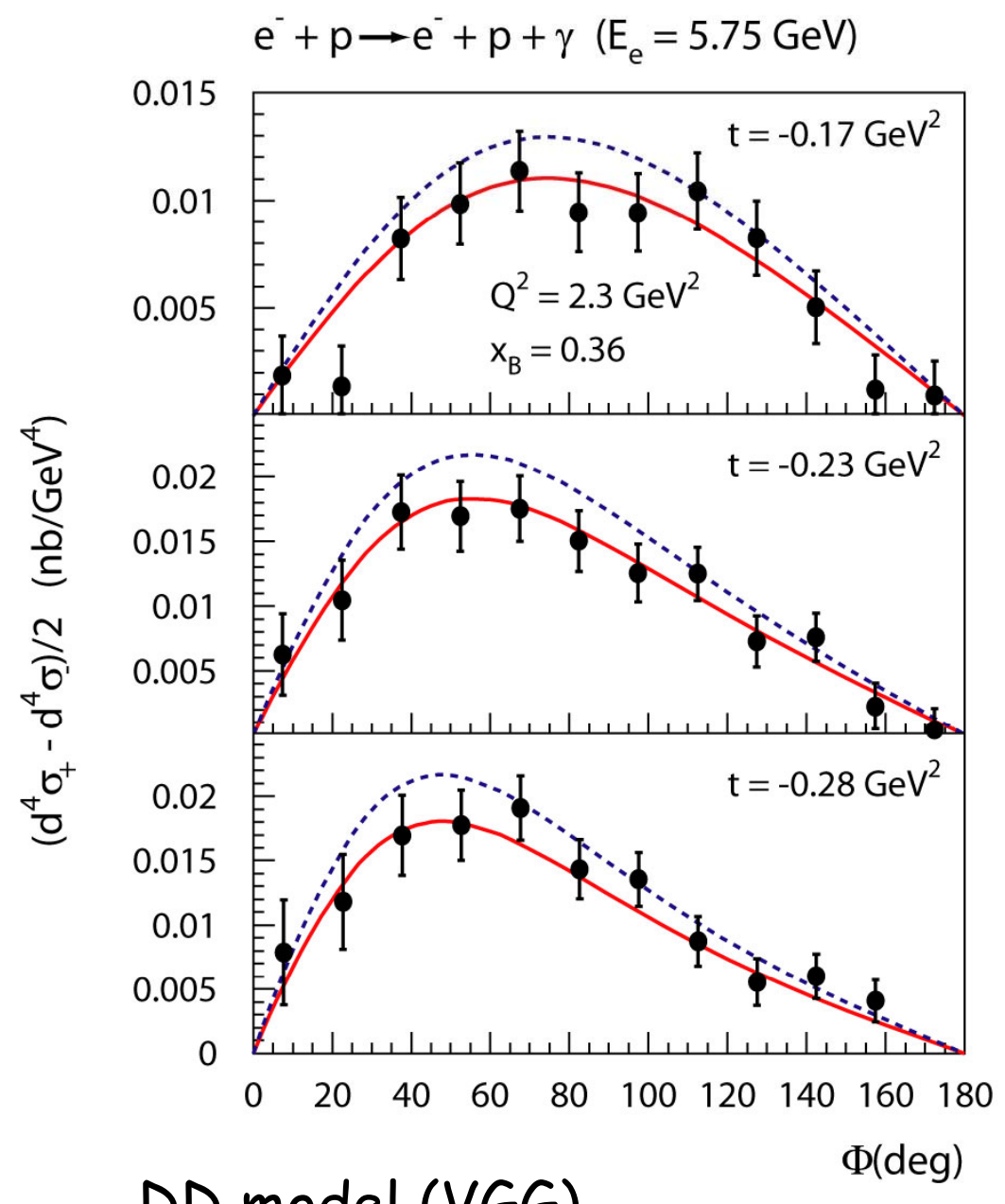

M. Polyakov, M. Vanderhaeghen arXiv:0803.1271

Data are perfectly described by minimal dual model

Minimal dual model

Data: Hall A polarized cross section data at $Q^{2}=2.3 \mathrm{GeV}^{2}$ 


\section{Minimal dual model (2)}

Beam Spin Asymmetries (Hall B data)

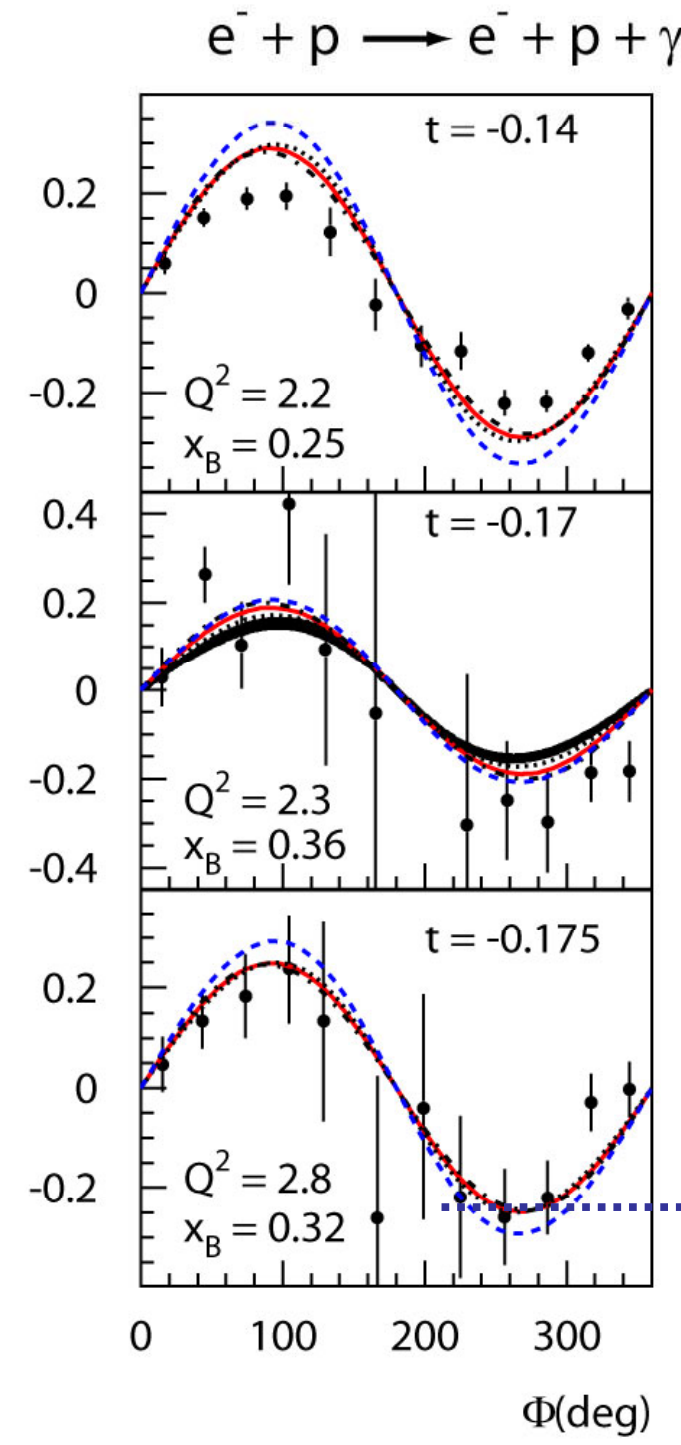

$\left(\mathrm{E}_{\mathrm{e}}=5.77 \mathrm{GeV}\right)$

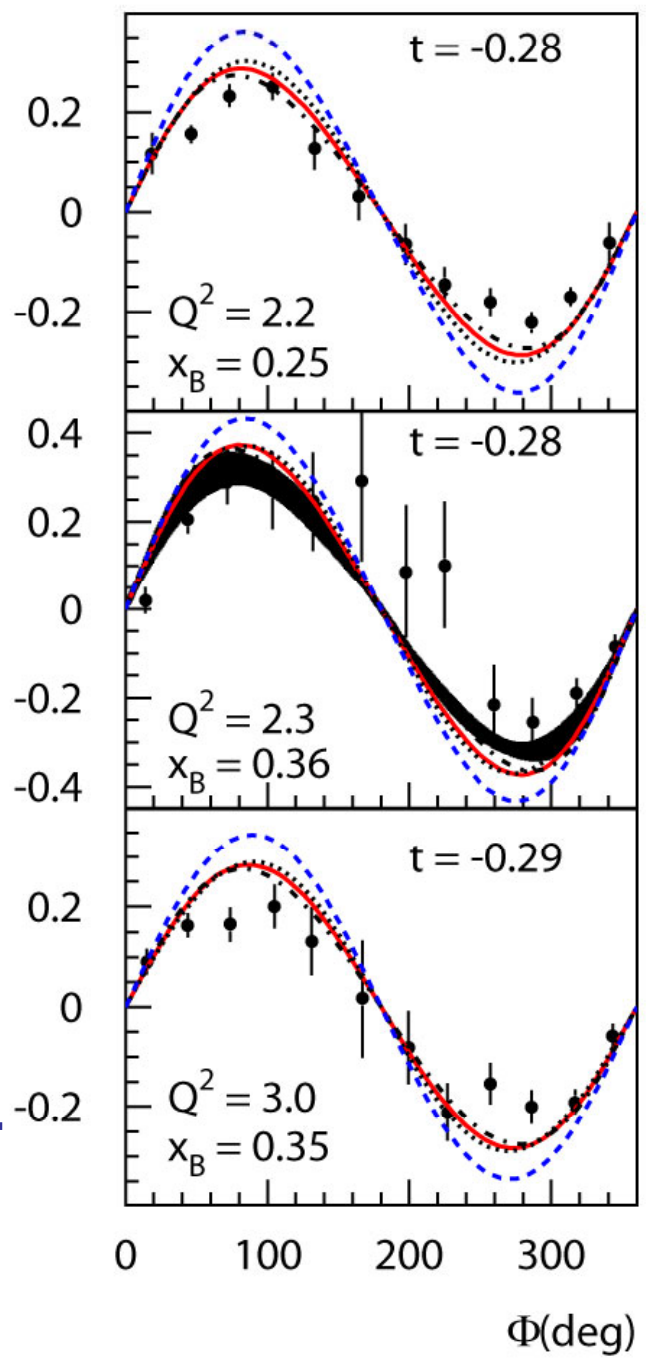

DD model ( $V G G$ )

Minimal dual model

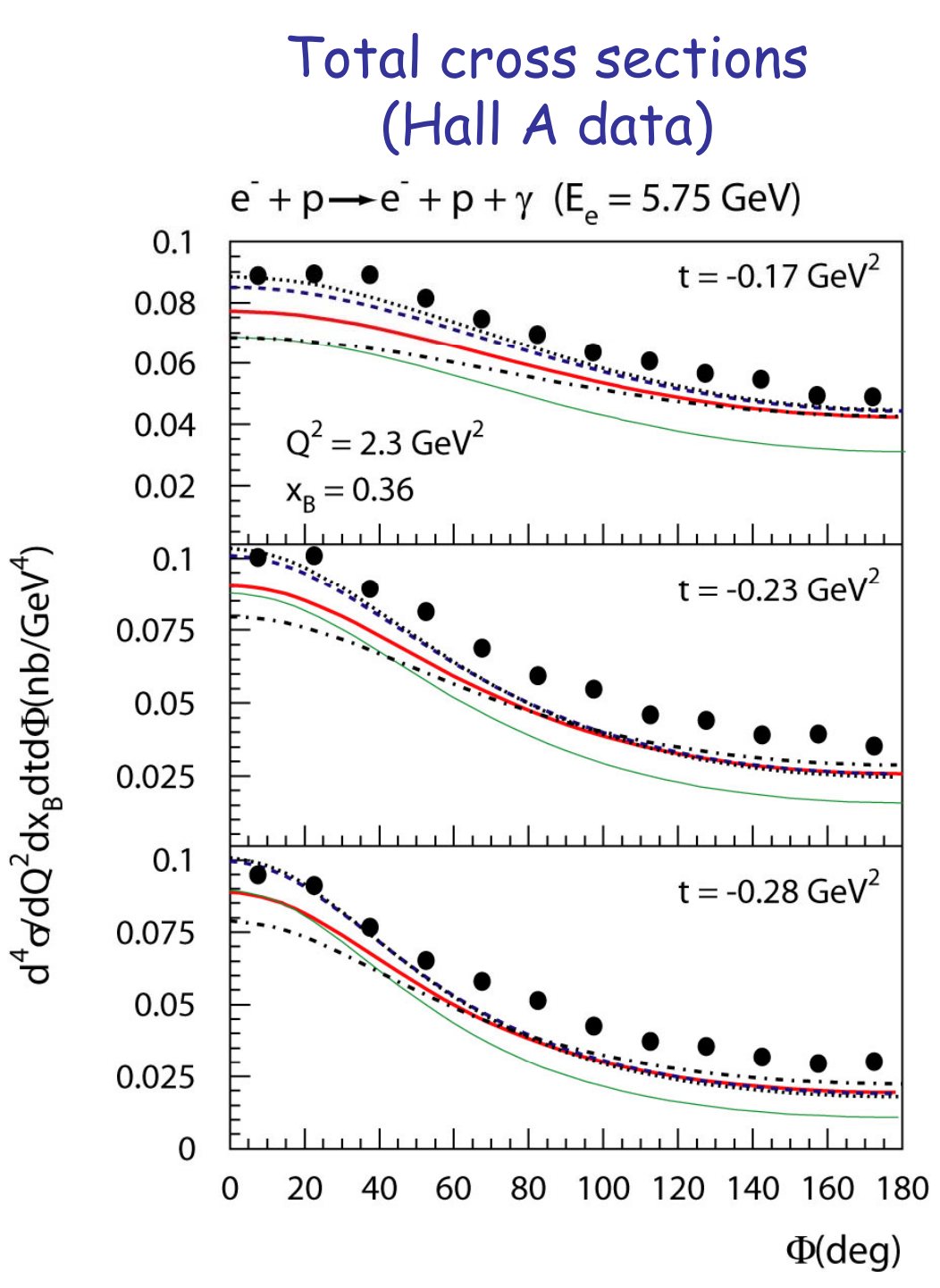




\section{Dispersion relation fits of DVCS data}

K. Kumericki, D. Mueller ArXiv: 0904.0458
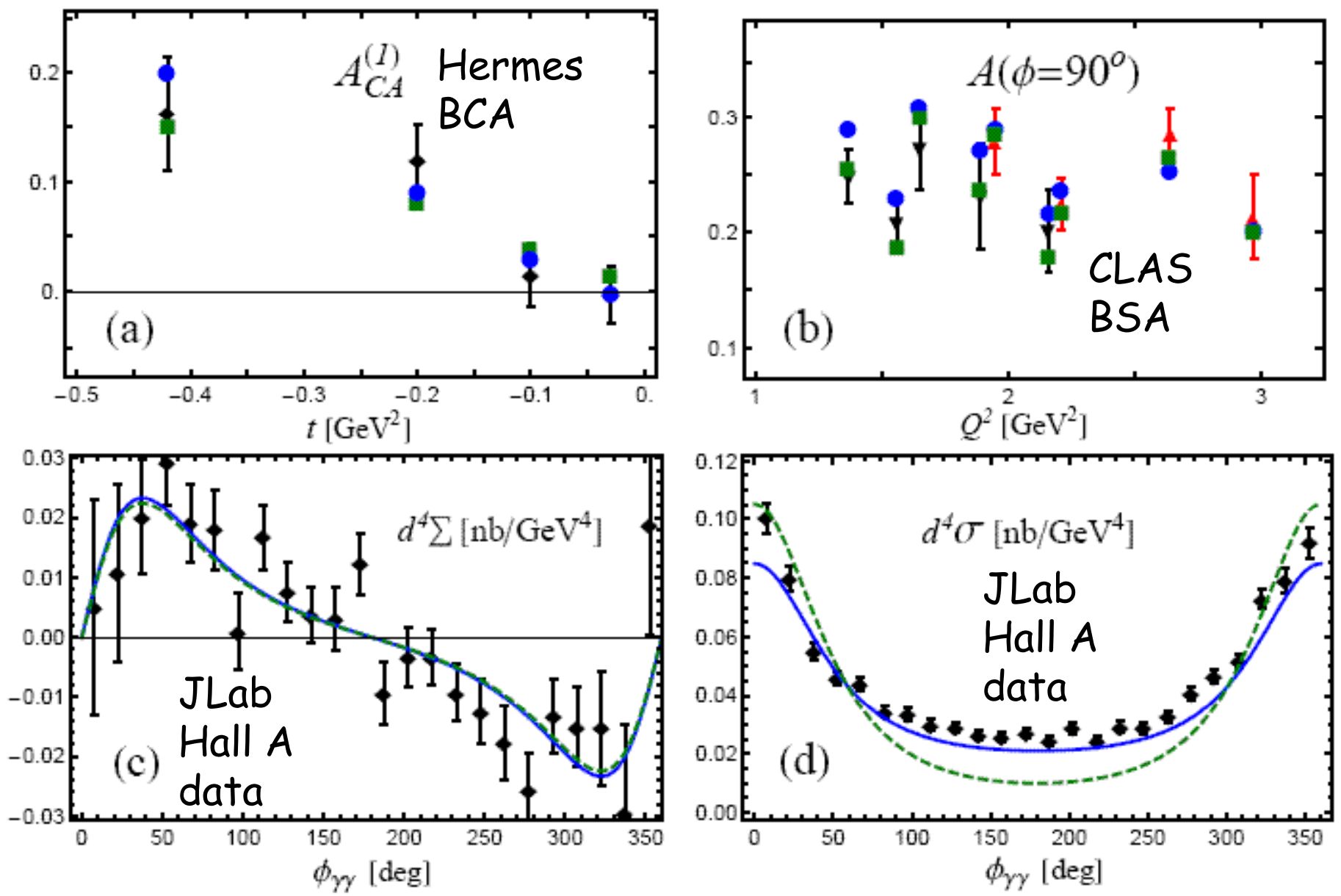

-......... Fit of H1/ZEUS+CLAS+Hermes with only $\mathrm{H}$

- + Hall $A$ and $H+\tilde{H}$ 


\section{Compton Form Factor (i.e. local) or dual model fits of $\mathrm{H}$ only}

Hall A cross section data

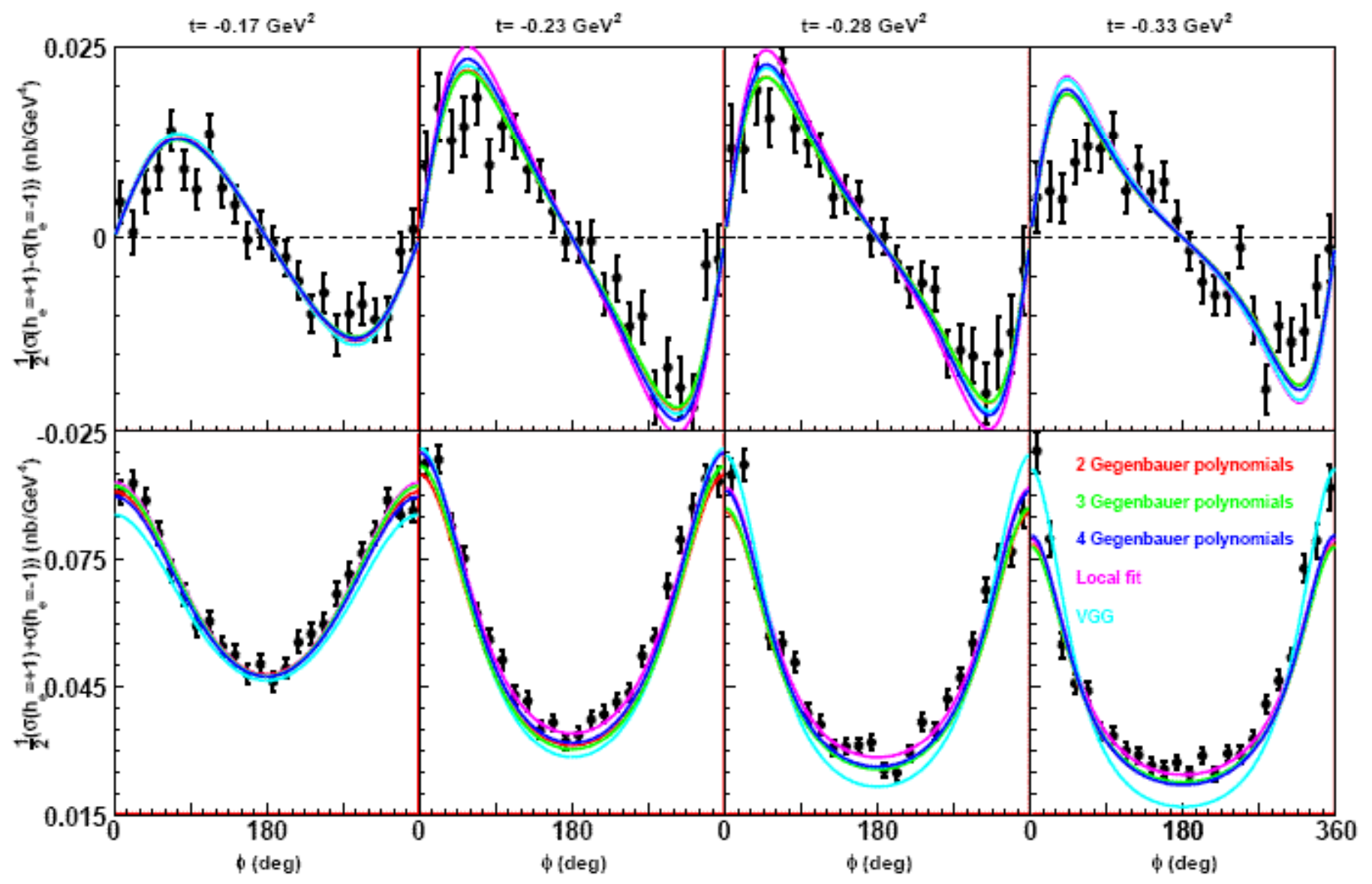

H. Moutarde

ArXiv: 0904.1648v1

To be published in Phys. Rev. D 


\section{Compton Form Factor fits only (i.e. local fits)}

Hall A cross section data $\quad \mathrm{E}_{\mathrm{e}}=5.75 \mathrm{GeV}, \mathrm{x}_{\mathrm{B}}=0.36, \mathrm{Q}^{2}=2.3 \mathrm{GeV}^{2}$

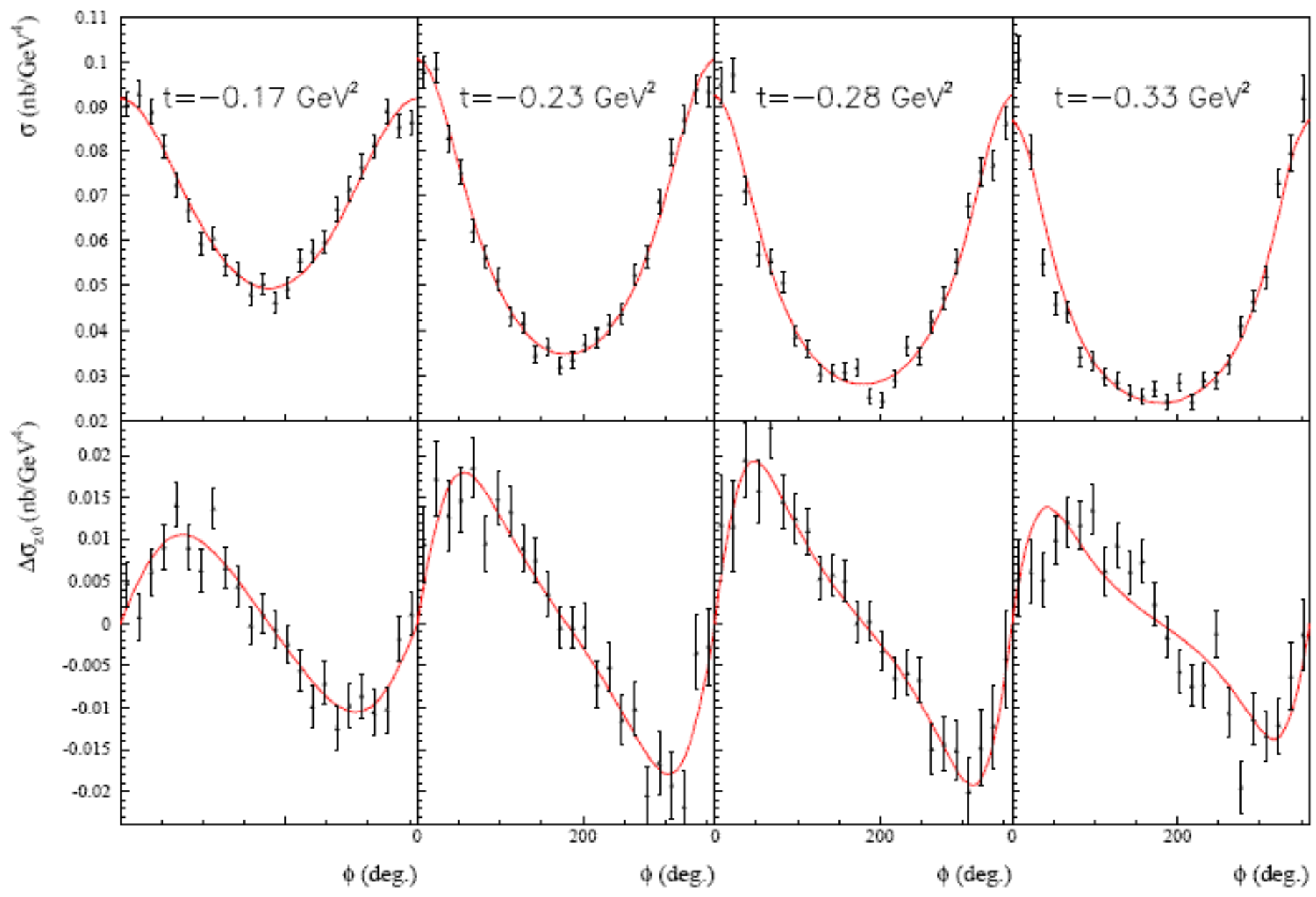

Includes $H, \tilde{H}$ and $E$

M. Guidal

Eur. Phys. J. A37 319, 2008 


\section{E1-DVCS : Cross-sections over a wide kinematical range}

PhD Thesis H.S. Jo
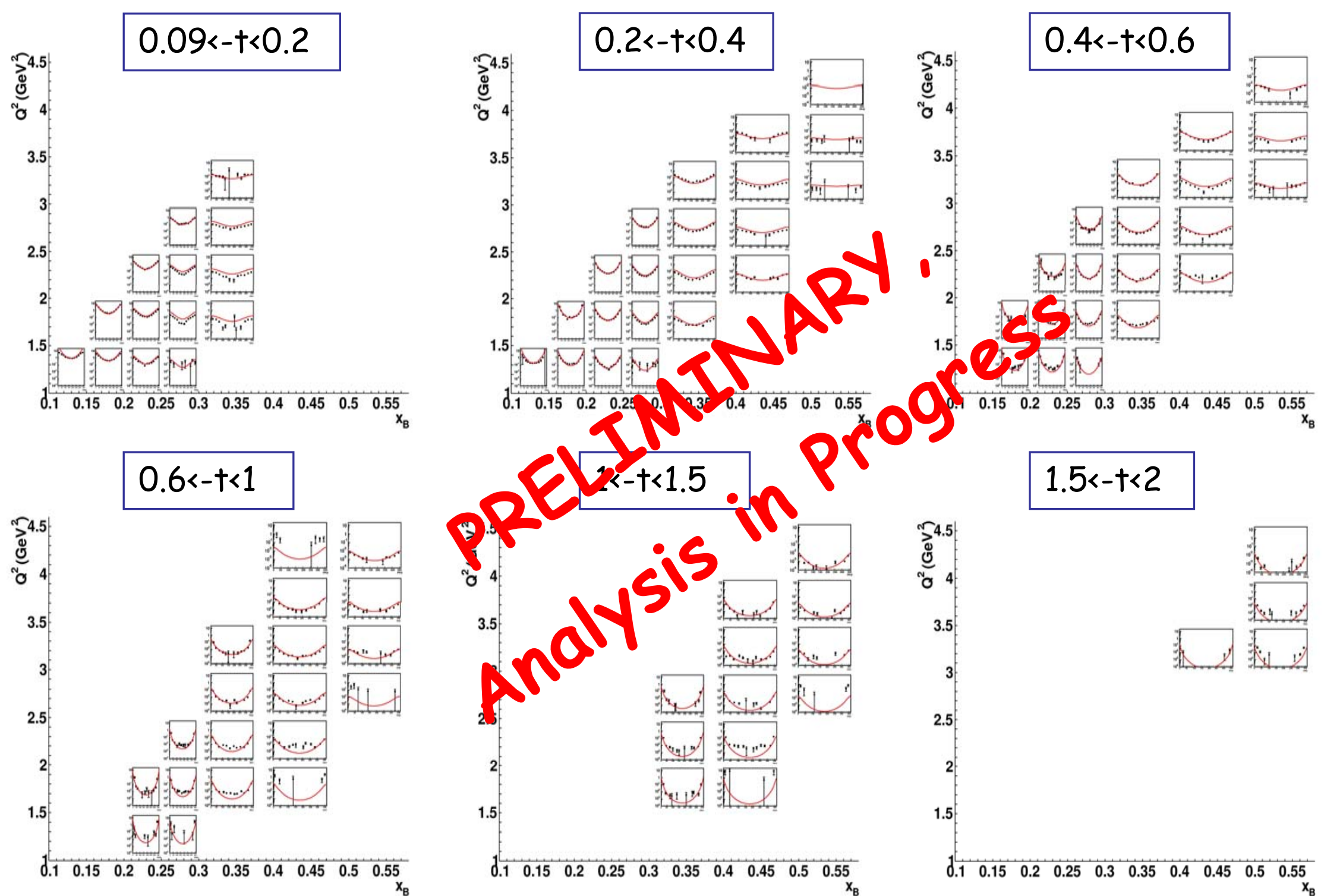


\section{$\pi^{0}$ electroproduction cross section}

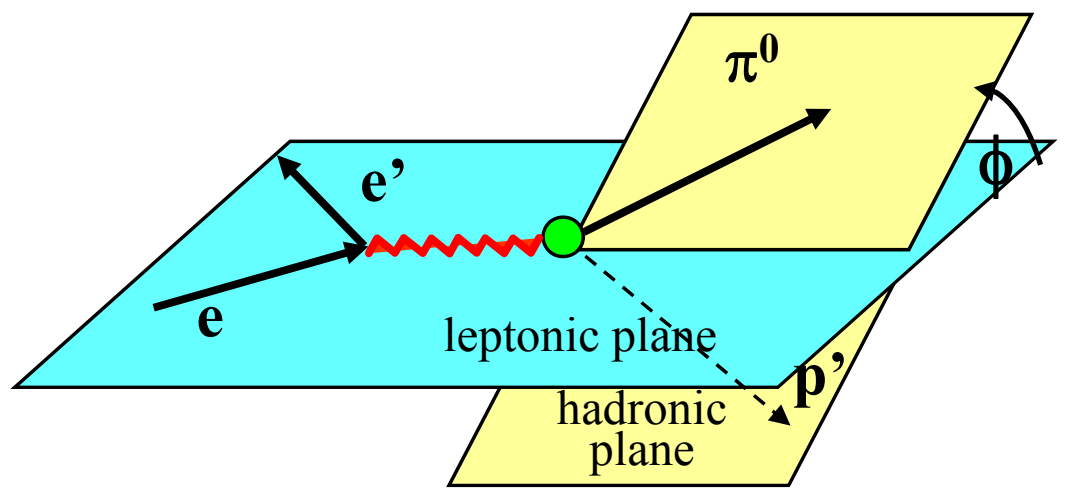

- $\mathrm{Q}^{2}=-\left(\mathrm{k}-\mathrm{k}^{\prime}\right)^{2}$

- $\mathrm{x}_{\mathrm{B}}=\mathrm{Q}^{2} / 2 \mathrm{Mv} \quad \mathrm{v}=\mathrm{E}_{\mathrm{e}}-\mathrm{E}_{\mathrm{e}}$,

$\cdot t=\left(p-p^{\prime}\right)^{2}$

- $\phi$ (angle between leptonic and hadronic plane)

$\frac{d^{4} \sigma}{d^{2} d_{B} d \phi d t}=\Gamma\left(Q^{2}, x_{B}\right) \frac{d^{2} \sigma}{d \phi d t} \quad$ reduced cross section for $\gamma^{*} \mathbf{p} \rightarrow \mathbf{p} \pi^{0}$

$\frac{\mathrm{d}^{2} \sigma}{\mathrm{d} \phi \mathrm{dt}}=\frac{1}{2 \pi}\left(\frac{\mathrm{d} \sigma_{\mathrm{T}}}{\mathrm{dt}}+\varepsilon \frac{\mathrm{d} \sigma_{\mathrm{L}}}{\mathrm{dt}}+\sqrt{2 \varepsilon(\varepsilon+1)} \frac{\mathrm{d} \sigma_{\mathrm{LT}}}{\mathrm{dt}} \cos \phi+\varepsilon \frac{\mathrm{d} \sigma_{\mathrm{TT}}}{\mathrm{dt}} \cos 2 \phi\right.$

$$
\left.+\mathrm{h} \sqrt{2 \varepsilon(\varepsilon-1)} \frac{\mathrm{d} \sigma_{\mathrm{L}}{ }^{\mathrm{T}} \mathrm{T}}{\mathrm{dt}} \sin \phi\right)
$$




\section{$\pi^{0}$ cross sections in Hall $A$ - a puzzle!}

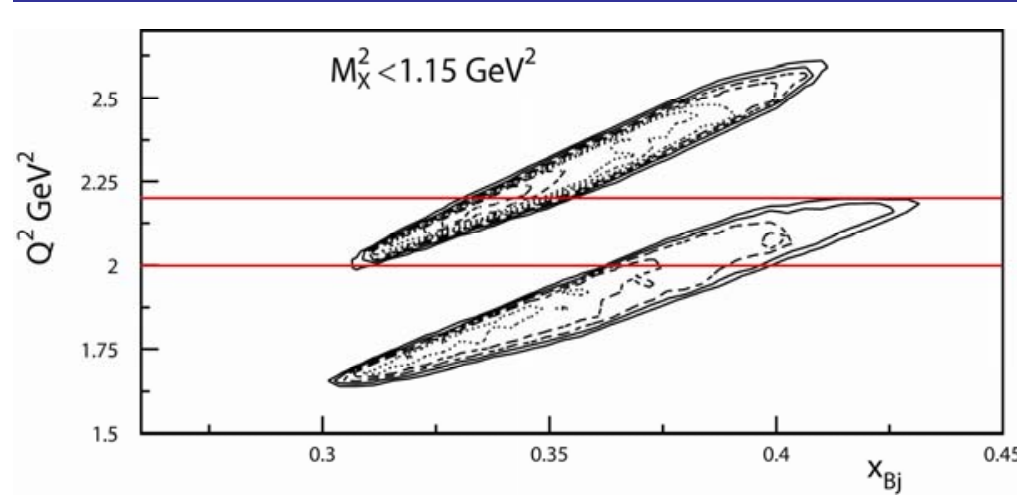

- Low- $\dagger$ cross-section largely overshoots GPD \& JML models !!!

-TT term is large, suggesting a large transverse component.

-But $\pi^{0}$ cross-section ratio for proton and deuteron targets is found $\sim 1$

More data needed!
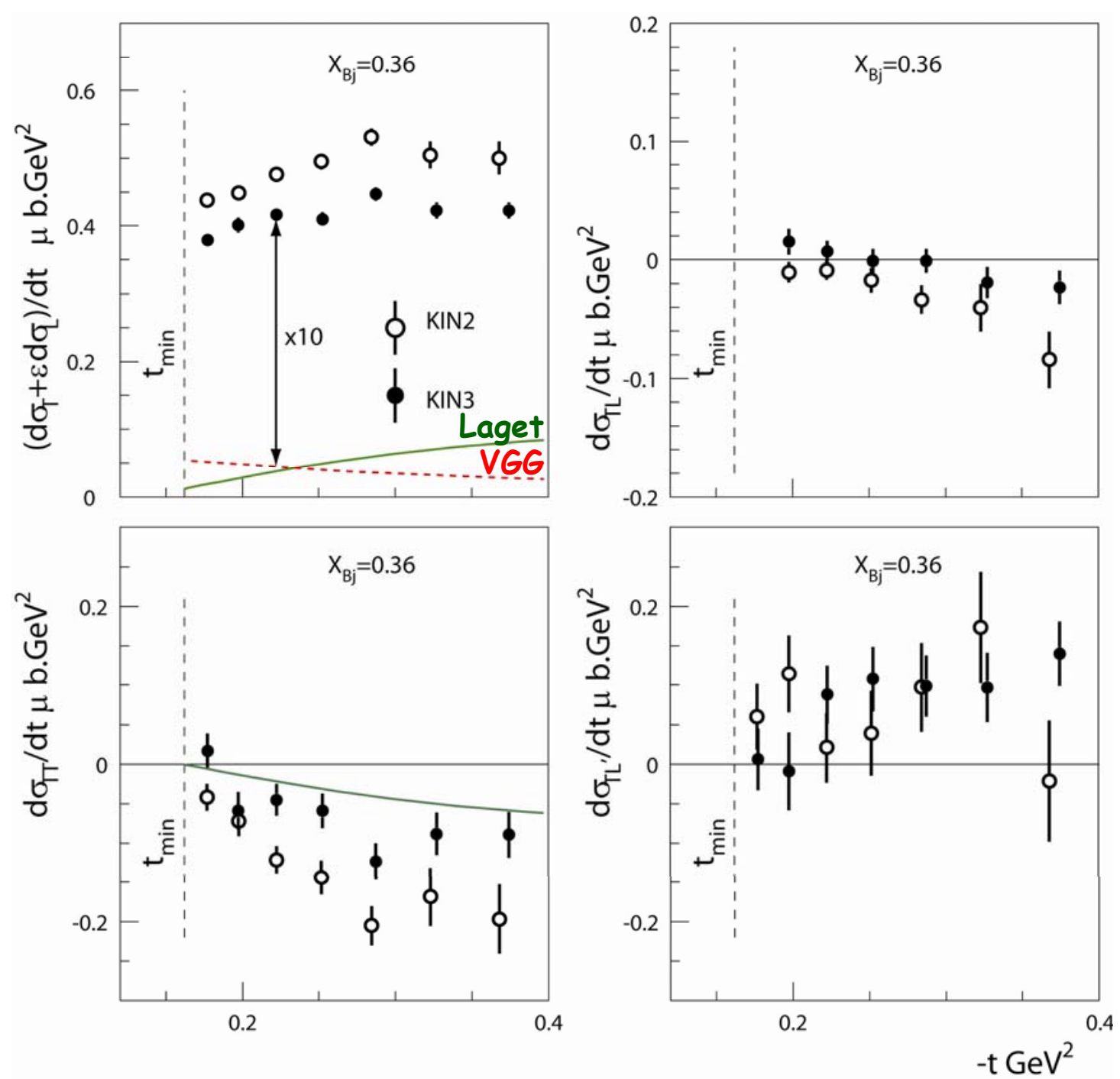


\section{$\pi^{0}$ cross sections in Hall B}

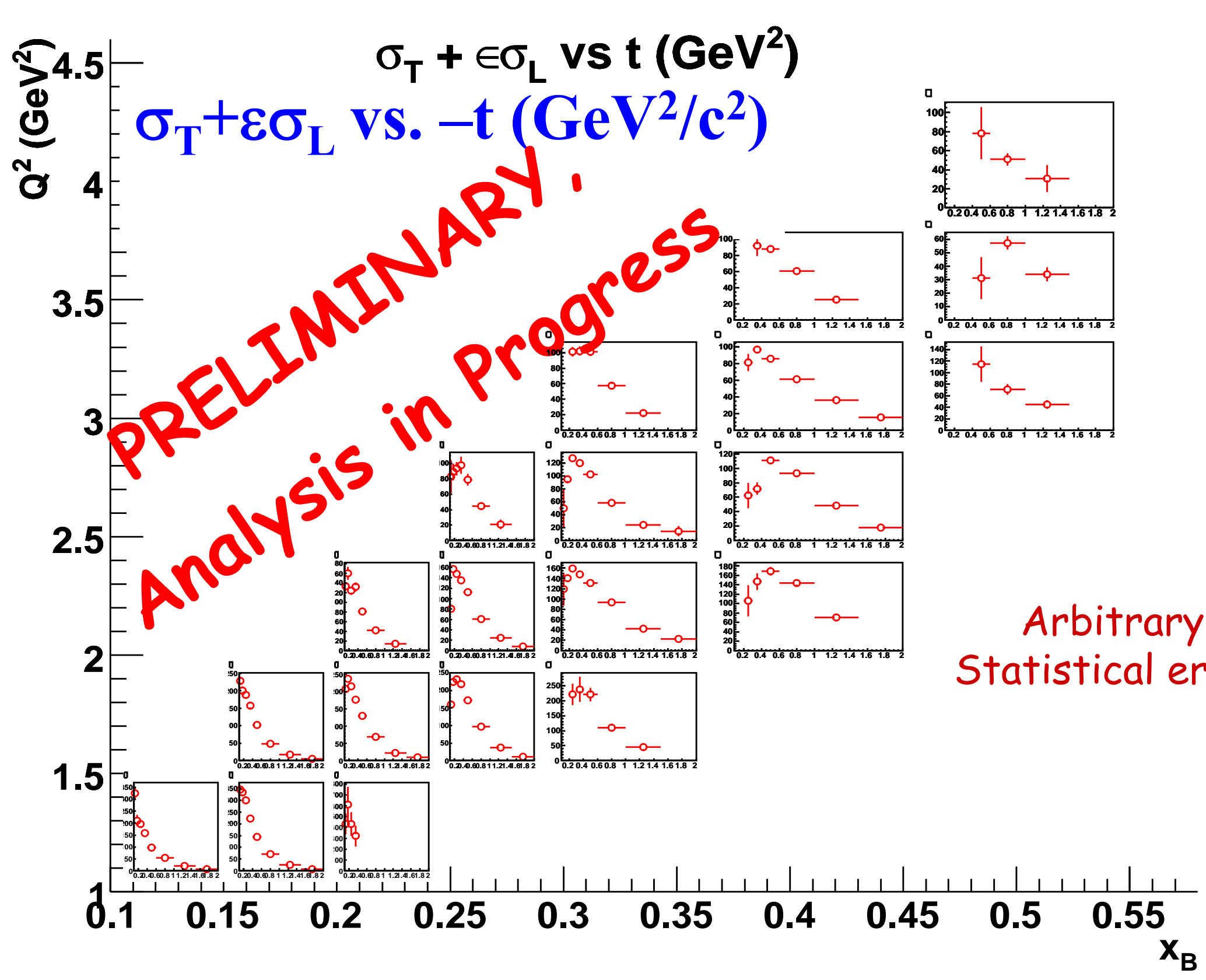

S. Niccolai 


\section{Experimental Setup and proposed experiments at $11 \mathrm{GeV}$}

Use of base CLAS12 equipment, including Inner Calorimeter (IC)

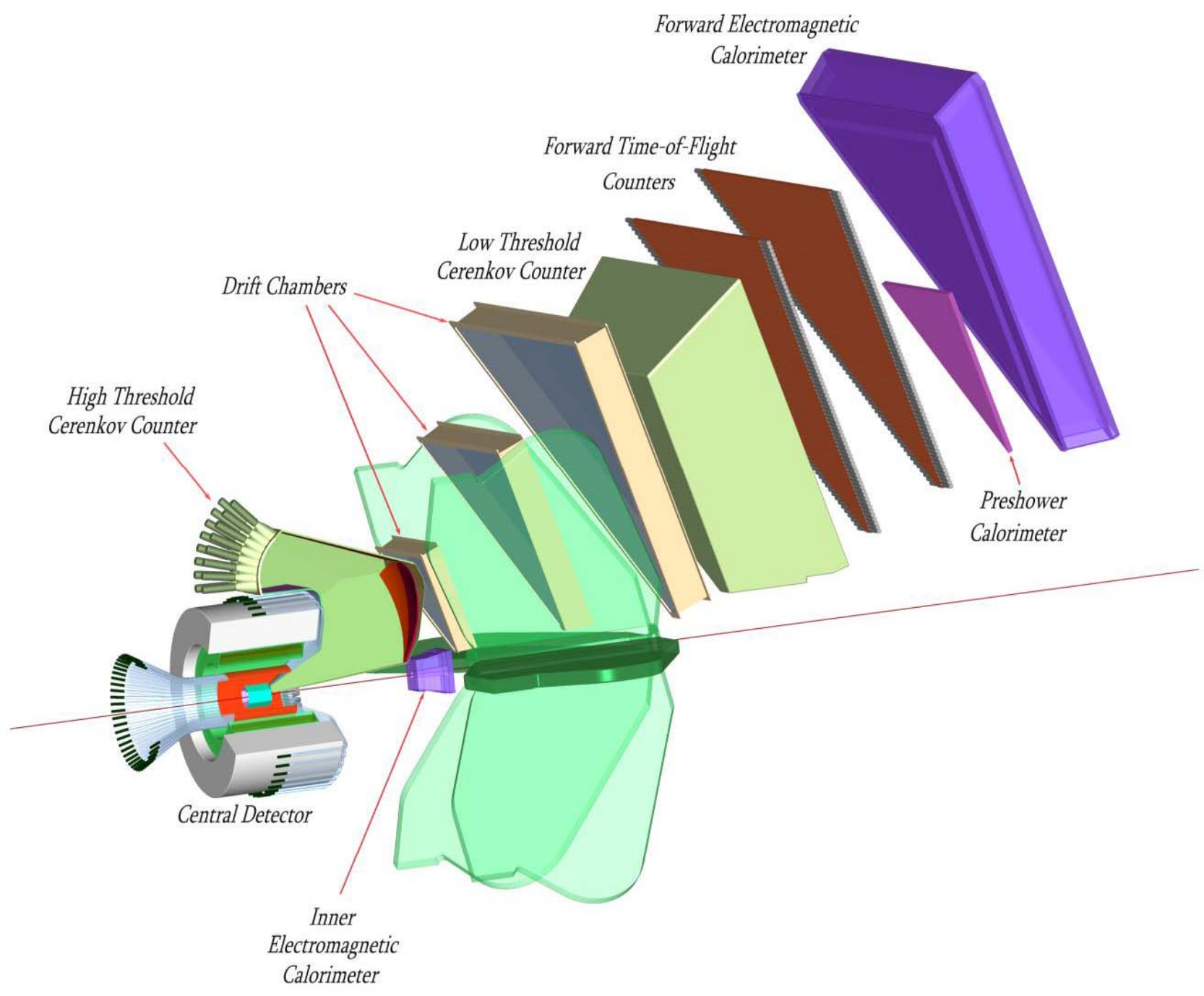




\section{Beam Spin Asymmetry}

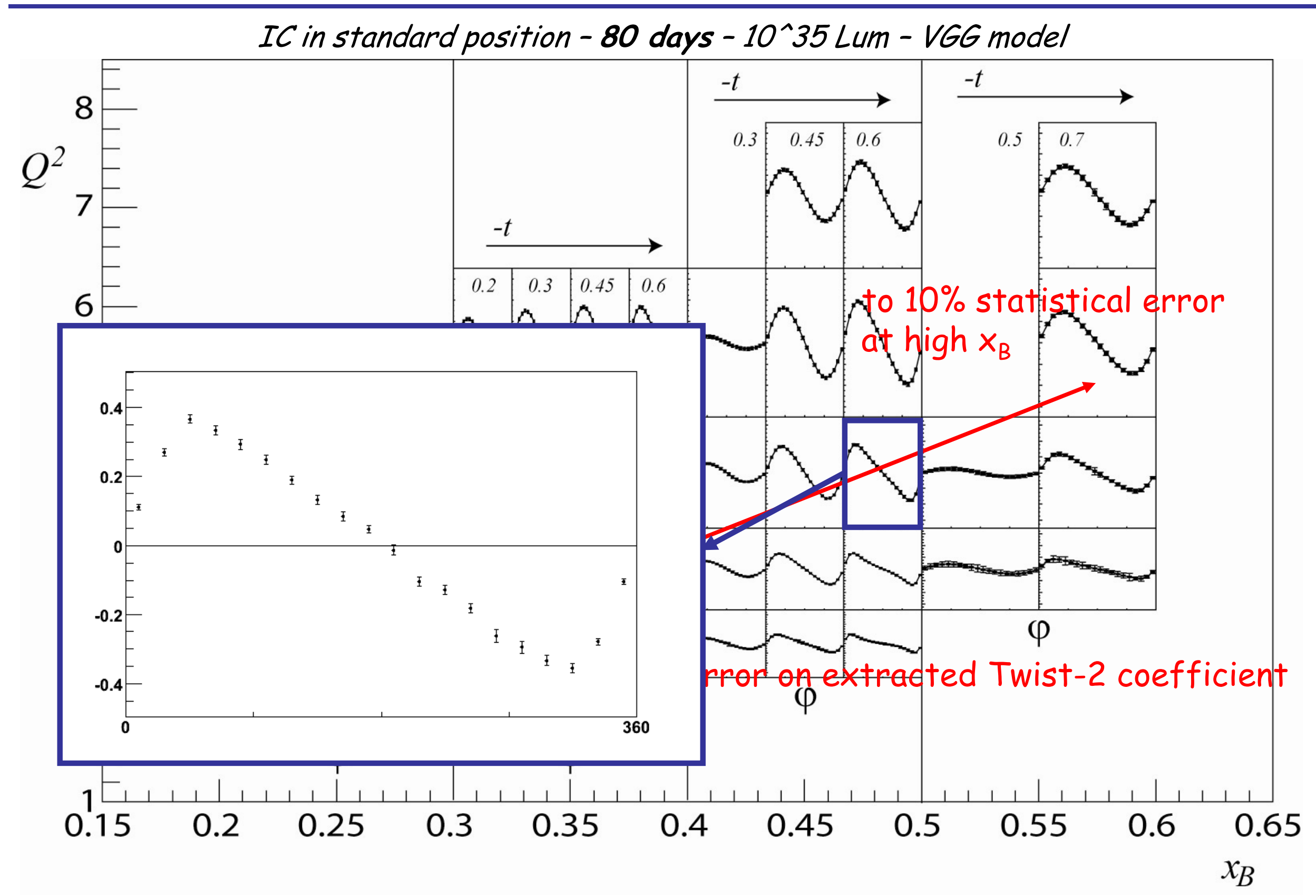




\section{Observables and their relationship to GPDs}

$T^{D V C S}=\int_{-1}^{+1} \frac{G P D(x, \xi, t)}{x-\xi+i \varepsilon} d x+\cdots$

The cross-section difference accesses the imaginary part of

DVCS and therefore GPDs at $x=\xi$

The total cross-section accesses the real part of DVCS and therefore an integral of GPDs over $x$

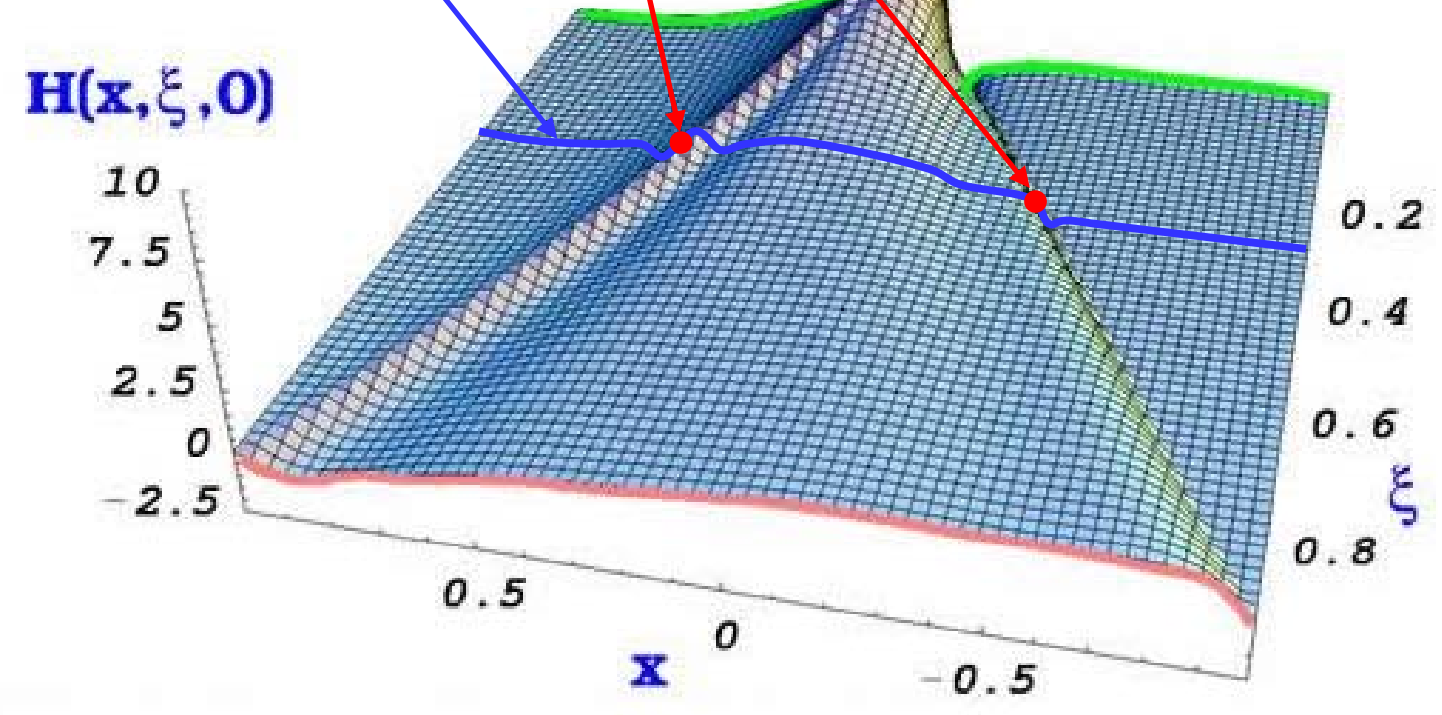




\section{(low/medium energy) Deep Exclusive experiments}

\begin{tabular}{|c|c|c|c|c|c|c|c|c|}
\hline $\begin{array}{l}\text { HERMES } \\
27 \mathrm{GeV}\end{array}$ & lished o & inalized & Publishe & $\begin{array}{l}\text { d or } \\
\text { lose to be } \\
\text { CLAS } \\
6 \mathrm{GeV}\end{array}$ & $\begin{array}{c}\text { Analysis in } \\
\text { progress }\end{array}$ & $\begin{array}{l}2009- \\
2010\end{array}$ & 2010+ & $\begin{array}{r}2014 ? \\
\text { JLab@ } \\
\text { 12GeV }\end{array}$ \\
\hline $\begin{array}{l}\text { DVCS - } \\
\text { BSA + BCA } \\
\frac{\text { nuclei }}{\text { BSA }} \\
\underline{\text { d-BCA }} \\
+\frac{\text { Polarized }}{\text { target }} \\
\text { DVCS } \\
\text { ep } \rightarrow \text { ep } \rho \\
\underline{\sigma_{L}}+\text { DSA }\end{array}$ & $\begin{array}{l}\text { DVCS } \\
\text { BSA }\end{array}$ & 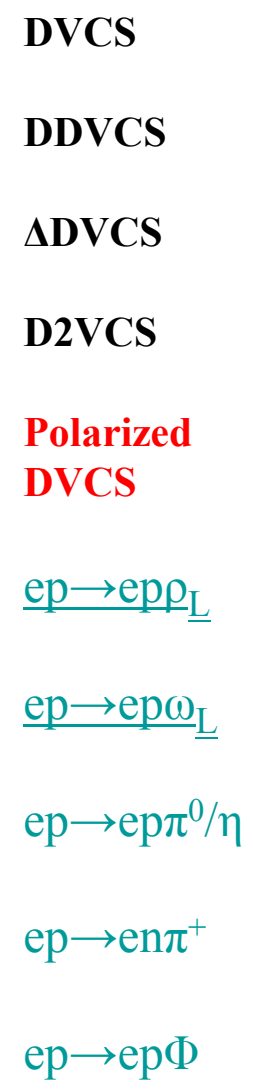 & $\begin{array}{l}\text { DVCS } \\
\text { proton } \\
\text { neutron }\end{array}$ & $\begin{array}{l}\text { DVCS } \\
\text { BSA } \\
\text { Proton } \\
\\
\text { ep } \rightarrow \mathbf{e p} \pi^{\mathbf{0}} / \eta\end{array}$ & $\begin{array}{l}\underline{\mathbf{D V C S}} \\
\underline{\mathbf{B S A}+\mathbf{B C A}} \\
\text { With recoil } \\
\text { detector }\end{array}$ & $\begin{array}{l}\text { Hall A } \\
\text { DVCS }^{2} \\
\text { CLAS } \\
\text { BSA+TSA }\end{array}$ & $\begin{array}{l}\underline{\text { DVCS }} \\
\underline{\text { G+BCA }} \\
\underline{\text { With recoil }} \\
\underline{\text { detector }}\end{array}$ & 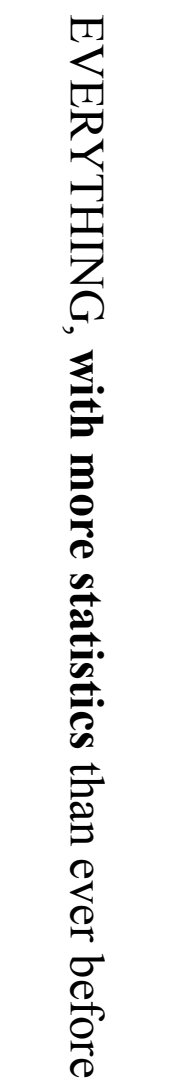 \\
\hline
\end{tabular}

In red: selected topics for JLab 